\title{
Orbiting Astronomical Satellite for Investigating Stellar Systems (OASIS): following the water trail from the interstellar medium to oceans
}

Christopher Walker, Gordon Chin, Susanne Aalto, Carrie Anderson, Jonathan Arenberg, et al.

Christopher K. Walker, Gordon Chin, Susanne Aalto, Carrie M. Anderson, Jonathan W. Arenberg, Cara Battersby, Edwin Bergin, Jenny Bergner, Nicolas Biver, Gordon L. Bjoraker, John Carr, Thibault Cavalié, Elvire De Beck, Michael A. DiSanti, Paul Hartogh, Leslie K. Hunt, Daewook Kim, Yuzuru Takashima, Craig Kulesa, David Leisawitz, Joan Najita, Dimitra Rigopoulou, Kamber Schwarz, Yancy Shirly, Antony A. Stark, Xander Tielens, Serena Viti, David Wilner, Edward Wollack, Erick Young, "Orbiting Astronomical Satellite for Investigating Stellar Systems (OASIS): following the water trail from the interstellar medium to oceans," Proc. SPIE 11820, Astronomical Optics: Design, Manufacture, and Test of Space and Ground Systems III, 1182000 (24 August 2021); doi: 10.1117/12.2594847

Event: SPIE Optical Engineering + Applications, 2021, San Diego, California, United States 


\title{
Orbiting Astronomical Satellite for Investigating Stellar Systems (OASIS): "Following Water from Galaxies, through Protostellar Systems, to Oceans"
}

\author{
Christopher K. Walker ${ }^{\mathrm{a}}$, Gordon Chin ${ }^{\mathrm{b}}$, Susanne Aalto ${ }^{\mathrm{c}}$, Carrie M. Anderson ${ }^{\mathrm{b}}$, Jonathan W. \\ Arenberg $^{\mathrm{d}}$, Cara Battersby ${ }^{\mathrm{e}}$, Edwin Bergin ${ }^{\mathrm{f}}$, Jenny Bergner ${ }^{\mathrm{g}, \mathrm{o}}$, Nicolas Biver ${ }^{\mathrm{h}}$, Gordon L. Bjoraker ${ }^{\mathrm{b}}$, \\ John Carri, Thibault Cavalié ${ }^{\mathrm{h}, \mathrm{j}}$, Elvire De Beck ${ }^{\mathrm{c}}$, Michael A. DiSanti ${ }^{\mathrm{b}}$, Paul Hartogh ${ }^{\mathrm{k}}$, Leslie K. \\ Hunt $^{1}$, Daewook Kim ${ }^{\mathrm{a}}$, Craig Kulesa ${ }^{\mathrm{a}}$, David Leisawitz ${ }^{\mathrm{b}}$, Joan Najita ${ }^{\mathrm{m}}$, Dimitra Rigopoulou ${ }^{\mathrm{n}}$, \\ Kamber Schwarz ${ }^{\mathrm{a}, \mathrm{o}}$, Yancy Shirly ${ }^{\mathrm{a}}$, Antony A. Stark ${ }^{\mathrm{p}}$, Yuzuru Takashima ${ }^{\mathrm{a}}$, Xander Tielens ${ }^{\mathrm{q}}$, Serena \\ Viti $^{\mathrm{q}, \mathrm{r}}$, David Wilner ${ }^{\mathrm{p}}$, Edward Wollack ${ }^{\mathrm{b}}$, and Erick Young ${ }^{\mathrm{s}}$ \\ ${ }^{a}$ University of Arizona, 950 N. Cherry Ave., Tucson, AZ USA \\ ${ }^{b}$ NASA 's Goddard Space Flight Center, 8800 Greenbelt Road, Greenbelt, MD USA \\ ${ }^{c}$ Department of Space, Earth and Environment with Onsala Space Observatory, Chalmers University of Technology, \\ SE-412 96 Göteborg, Sweden \\ ${ }^{d}$ Northrop Grumman Space Systems, One Space Park Drive, Redondo Beach, CA USA \\ ${ }^{e}$ University of Connecticut, Department of Physics, 196 Auditorium Road, Unit 3046, Storrs, CT 06269 USA \\ ${ }^{f}$ University of Michigan, Department of Astronomy, 1085 S. University, Ann Arbor, MI USA \\ ${ }^{g}$ Department of the Geophysical Sciences, University of Chicago, 5734 S. Ellis Avenue Chicago, Illinois \\ ${ }^{h}$ LESIA, Observatoire de Paris, PSL Research University, CNRS, Sorbonne Universités, UPMC Univ. Paris 06, Univ. \\ Paris Diderot, Sorbonne Paris Cité, Meudon, France \\ ${ }^{i}$ Astronomy Department, University of Maryland, College Park, MD 20742-2421 USA \\ ${ }^{j}$ Laboratoire d'Astrophysique de Bordeaux, Univ. Bordeaux, CNRS, B18N, Allée Geoffroy Saint-Hilaire, 33615 Pessac, \\ France \\ ${ }^{k}$ Max Planck Institute for Solar System Research, Justus-von-Liebig-Weg 3, Göttingen, Germany

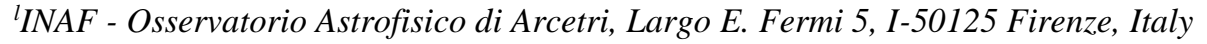 \\ ${ }^{m}$ NSF's NOIRLab, 950 N. Cherry Avenue, Tucson, AZ 85719, USA \\ ${ }^{n}$ Department of Physics, University of Oxford, Keble Road, Oxford OX1 3RH, UK \\ ${ }^{\circ}$ NASA Fellowship Program Sagan Fellow \\ ${ }^{p}$ Center for Astrophysics Harvard \& Smithsonian, 60 Garden Street, Cambridge, MA, USA 02138 \\ ${ }^{q}$ Leiden Observatory, Leiden University, P.O. Box 9513, NL-2300RA Leiden, The Netherlands \\ ${ }^{r}$ Department of Physics and Astronomy, University College London, Gower St., WC1 6BT, London, UK \\ ${ }^{s}$ Universities Space Research Association, 15 National Ave, Mountain View, CA USA
}

\begin{abstract}
Orbiting Astronomical Satellite for Investigating Stellar Systems $(O A S I S)$ is a space-based, MIDEX-class mission concept that employs a 17-meter diameter inflatable aperture with cryogenic heterodyne receivers, enabling high sensitivity and high spectral resolution (resolving power $\geq 10^{6}$ ) observations at terahertz frequencies. OASIS science is targeting submillimeter and far-infrared transitions of $\mathrm{H}_{2} \mathrm{O}$ and its isotopologues, as well as deuterated molecular hydrogen (HD) and other molecular species from 660 to $80 \mu \mathrm{m}$, which are inaccessible to ground-based telescopes due to the opacity of Earth's atmosphere. OASIS will have $>20 \mathrm{x}$ the collecting area and $\sim 5 \mathrm{x}$ the angular resolution of Herschel, and it complements the shorter wavelength capabilities of the James Webb Space Telescope. With its large collecting area and suite of terahertz heterodyne receivers, OASIS will have the sensitivity to follow the water trail from galaxies to oceans, as well as directly measure gas mass in a wide variety of astrophysical objects from observations of the ground-state HD line. OASIS will operate in a Sun-Earth L1 halo orbit that enables observations of large numbers of galaxies, protoplanetary systems, and solar system objects during the course of its 1-year baseline mission. OASIS embraces an overarching science
\end{abstract}

Astronomical Optics: Design, Manufacture, and Test of Space and Ground Systems III, edited by Tony B. Hull, Daewook Kim, Pascal Hallibert, Fanny Keller, Proc. of SPIE Vol. 11820, 1182000 - ( ) The Authors.

Published under a Creative Commons Attribution CC-BY 3.0 License $\cdot$ doi: 10.1117/12.2594847 
theme of "following water from galaxies, through protostellar systems, to oceans." This theme resonates with the NASA Astrophysics Roadmap and the 2010 Astrophysics Decadal Survey, and it is also highly complementary to the proposed Origins Space Telescope's objectives.

Keywords: Water, HD, terahertz astronomy, submillimeter spectroscopy, far-infrared spectroscopy, heterodyne spectroscopy, galaxies, proto-planetary disks, comets, planets, moons

\section{INTRODUCTION}

\subsection{OASIS Pioneers New Era of Large-Aperture Space Telescopes}

The Orbiting Astronomical Satellite for Investigating Stellar Systems (OASIS) observatory utilizes an inflatable 17-m reflector that, when coupled to a state-of-the-art terahertz heterodyne receiver system, enables high sensitivity and high spectral resolution (resolving power $\geq 10^{6}$ ) observations at terahertz frequencies. This ensures submillimeter and farinfrared observations of the transitions of $\mathrm{H}_{2} \mathrm{O}$ and its isotopologues, as well as deuterated molecular hydrogen (HD) and other molecular species from 660 to $80 \mu \mathrm{m}$ that are otherwise obscured by the Earth's atmosphere. As a result, the OASIS science team, internationally-comprised of extra-galactic, galactic, and planetary astronomers, are able to follow the water trail from galaxies, through protostellar systems, to oceans (see Figure 1). OASIS will have $>20 \mathrm{x}$ the collecting area and $\sim 5 \mathrm{x}$ the angular resolution of the Herschel Space Observatory. Our team will propose OASIS in response to the planned 2021 NASA MIDEX Announcement of Opportunity (AO), thus breaking the paradigm that large-aperture space telescopes can only be implemented as a high-cost, long lead-time, flagship mission (e.g., JWST).

The use of an inflatable membrane aperture as a reflector has a long history of space applications. As described by [1], an inflatable membrane mirror can be formed by using two thin, circular, polymer membranes (one transparent and the other metallized) that are sealed on their periphery, attached to a tensioning ring and inflated to a pressure sufficient to produce the required shape. Work performed under NASA's Innovative Advanced Concepts (NIAC) program (as well as other federal agencies) has demonstrated that thin membrane technology combined with adaptive optics techniques can be used to realize large, deployable reflectors for operation throughout the far-IR. Over 50 years ago Project Echo (30.5-m

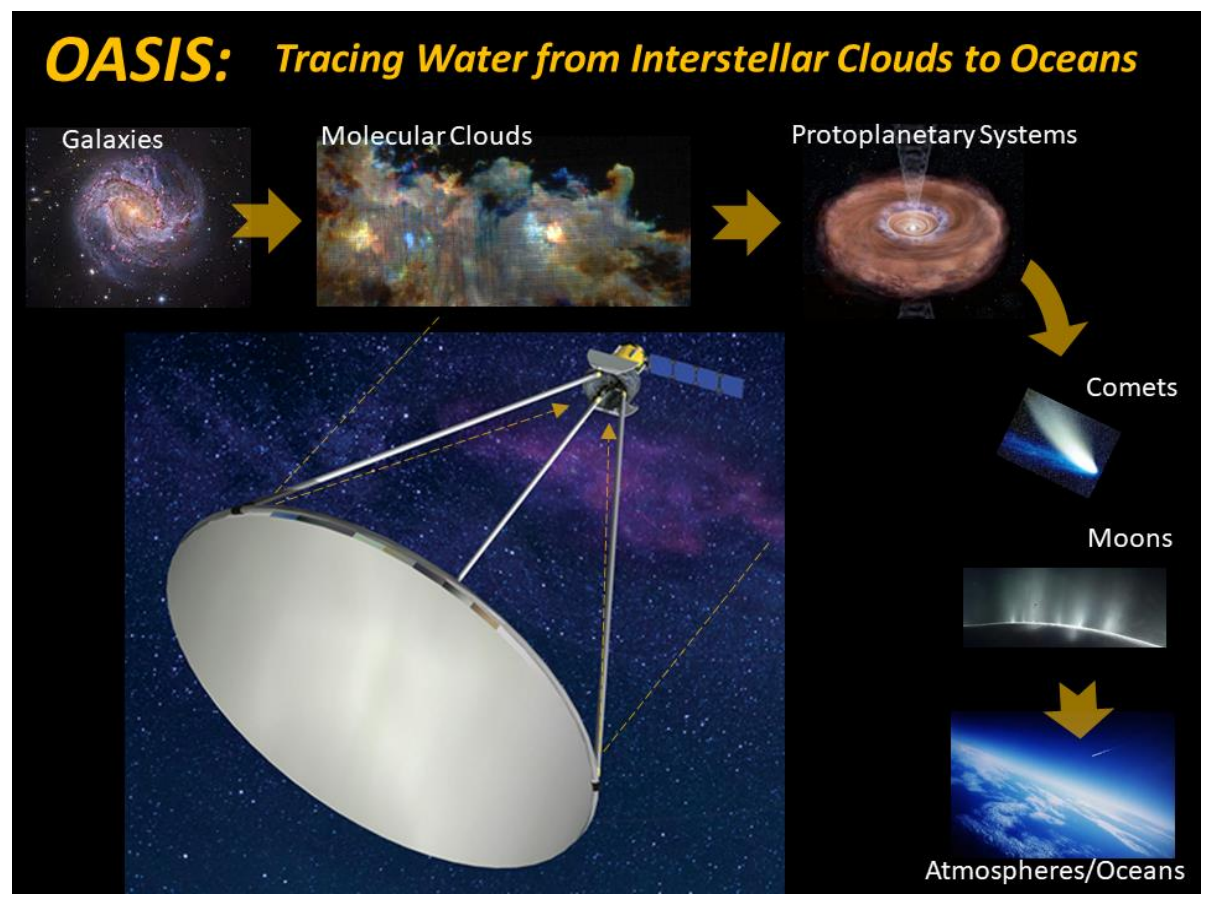

Figure 1. OASIS Concept. The science objectives of OASIS are met by utilizing a 17 -meter inflatable aperture and cryogenic, terahertz receivers operating in a Sun-Earth L1 Halo Orbit. From this vantage point, the unparalleled sensitivity of $O A S I S$ will, for the first time, allow us to follow the water trail from galaxies to oceans. 
diameter; [2]) and, more recently, the Inflatable Aperture Experiment (IAE) (14-m; diameter [3]), provided valuable onorbit experience with the fabrication and deployment of large inflatable reflectors.

In the hard vacuum of space, the internal pressures required to inflate and maintain such structures are very low $\left(<10^{-4}\right.$ psi), which makes them resilient against punctures from micrometeorites and space debris. Due to these low pressures, gas only slowly diffuses through punctures. The OASIS team has performed experiments with an inflatable structure in a vacuum chamber, demonstrating this unique property (See Paper No 11820-31 in Table 1). Initial lifetime calculations have been performed assuming a standard micrometeorite background flux, with the result that multiyear operation can be achieved by carrying a modest amount of inflatant gas to maintain pressure even with an accumulation of punctures over the lifetime of a mission that may last several years. Due to less surface stress being required to maintain the reflector's shape, larger apertures require lower internal pressures, which means less inflatant is needed per unit volume. In this respect, it is easier to make larger apertures than smaller ones using this type of technology.

This paper details the powerful science capabilities attainable with the high sensitivity ( 20x the collecting area of Herschel) and high angular resolution (5x that of Herschel) afforded by the large OASIS aperture. The OASIS team has submitted a series of papers to the 2021 SPIE Optics + Photonics Conference in San Diego, which collectively present the $O A S I S$ scientific goals and technical studies that have been performed in support of the mission (Table 1). These studies will serve as catalysts for future efforts.

Table 1. OASIS Papers Submitted to the Proceedings of the 2021 SPIE Optics + Photonics Conference are references for the OASIS Observatory architecture and trade studies.

\begin{tabular}{|l|l|l|}
\hline Paper No & \multicolumn{1}{|c|}{ Title } & \multicolumn{1}{|c|}{ Authors } \\
\hline $11820-26$ & $\begin{array}{l}\text { Orbiting Astronomical Satellite for Investigating Stellar Systems (OASIS): } \\
\text { Following Water from Galaxies and Interstellar Medium to Oceans }\end{array}$ & Christopher K. Walker, et al. \\
\hline $11820-27$ & $\begin{array}{l}\text { All reflective THz telescope design with a 20m inflatable primary antenna } \\
\text { for Orbiting Astronomical Satellite for Investigating Stellar Systems } \\
\text { (OASIS) mission }\end{array}$ & Yuzuru Takashima, et al. \\
\hline $11820-28$ & $\begin{array}{l}\text { Parametric design study of all reflective 20m telescope with an inflatable } \\
\text { primary antenna for Orbiting Astronomical Satellite for Investigating } \\
\text { Stellar Systems (OASIS) }\end{array}$ & Siddhartha Sirsi, et al. \\
\hline $11820-29$ & $\begin{array}{l}\text { Stressed Deformable Reflector for Terahertz Wavefront Active Correction } \\
11820-30\end{array} \begin{array}{l}\text { Orbiting Astronomical Satellite for Investigating Stellar Systems (OASIS) } \\
\text { observatory design }\end{array}$ & Jonathan W. Arenberg, et al. \\
\hline $11820-31$ & $\begin{array}{l}\text { Design and performance of the Orbiting Astronomical Satellite for } \\
\text { Investigating Stellar Systems (OASIS) }\end{array}$ & Jonathan W. Arenberg, et al. \\
\hline $11820-32$ & $\begin{array}{l}\text { Analytical and finite element analysis tool for nonlinear membrane antenna } \\
\text { modeling for astronomical applications }\end{array}$ & Art Palisoc, et al. \\
\hline $11820-33$ & Mid to high frequency characterization of inflatable membrane optics & Heejoo Choi, et al. \\
\hline $11820-34$ & Pre-flight optical metrology for varifocal pneumatic terahertz antenna & Henry Quach, et al. \\
\hline
\end{tabular}

\subsection{OASIS Unique Capabilities}

OASIS embraces an overarching science theme of "following water from galaxies, through protostellar systems, to oceans." This theme requires space-borne observations of galaxies, molecular clouds, protoplanetary disks, and solar system objects. From its Sun-Earth L1 halo orbit, OASIS will also be able to study the Earth-Moon system, which will serve as a surrogate from which we can learn how to identify and interpret the appearance of biosignatures on more distant worlds.

The $0.4-4 \mathrm{THz}$ spectral range that OASIS is targeting contains key diagnostic spectral fingerprints of HD and the isotopologues of $\mathrm{H}_{2} \mathrm{O}$ that are obscured by the Earth's atmosphere. Figure 2 shows a ground-based transmission spectrum of Earth's atmosphere for an elevation of $5 \mathrm{~km}$ (i.e., for ALMA) under dry conditions. The tuning ranges of OASIS Bands 
1,2, and 3, and the narrow Band 4 are indicated. These bands are extremely rich in spectral content, with strong rotational transitions of $\mathrm{H}_{2} \mathrm{O}$ isotopologues and numerous other molecules. The extreme opacity of Earth's atmosphere in the submillimeter and far-IR is the reason that ALMA (limited to frequencies below $\sim 1 \mathrm{THz}$ ) has made very few measurements of $\mathrm{HDO}$ and $\mathrm{H}_{2}{ }^{18} \mathrm{O}[4,5]$. This reinforces the need for a space-borne platform with instruments operating in these spectral regions. OASIS was designed specifically for this purpose.

\subsection{OASIS Instrument}

OASIS will have a cryogenic superheterodyne receiver system with 4 frequency bands ranging from $455 \mathrm{GHz}(660 \mu \mathrm{m})$ to $3692 \mathrm{GHz}(81.2 \mu \mathrm{m})$. The receiver architecture is shown in Figure 3. The OASIS receiver design is based on the instrument to be flown on the upcoming Galactic/Extragalactic ULDB Spectroscopic Terahertz Observatory (GUSTO) Explorer balloon-borne mission, and the instrument successfully flown in 2016 on the Stratospheric Terahertz Observatory (STO) balloon-borne telescope. The beam enters the receiver system from the steering and correction optics (described in submitted papers listed in Table 1) and is spectrally split by a series of dichroics into the 4 science bands. For Bands 2-4, the local oscillator (LO) beams are coupled to the respective science beam using dielectric beam splitters. Band 1 utilizes a waveguide coupler for this purpose. The four beams propagate through the cryostat into their respective front-end mixers. The mixers downconvert the science signals to intermediate frequencies (IF) in the microwave range where cryogenic low-noise amplifiers (LNAs) and ambient-temperature IF processors are used to boost their power levels and provide filtering before they are passed to their respective spectrometers. The resulting spectra are then conveyed to the spacecraft for downlink. The total power output over the full IF bandwidth of each receiver $(\sim 3.7 \mathrm{GHz})$ is also provided. For calibration, the receiver beam is diverted to a calibration blackbody cone by actuating a flip mirror. Additionally, a bolometer with a $2.63 \mathrm{THz}(114 \mu \mathrm{m})$ to $3.57 \mathrm{GHz}(84 \mu \mathrm{m})$ passband is used for tracking the target. While the four science bands observe the calibration load, the bolometer observes the astrophysical target off the back of the flip mirror. Table 2 is a summary of OASIS performance characteristics.

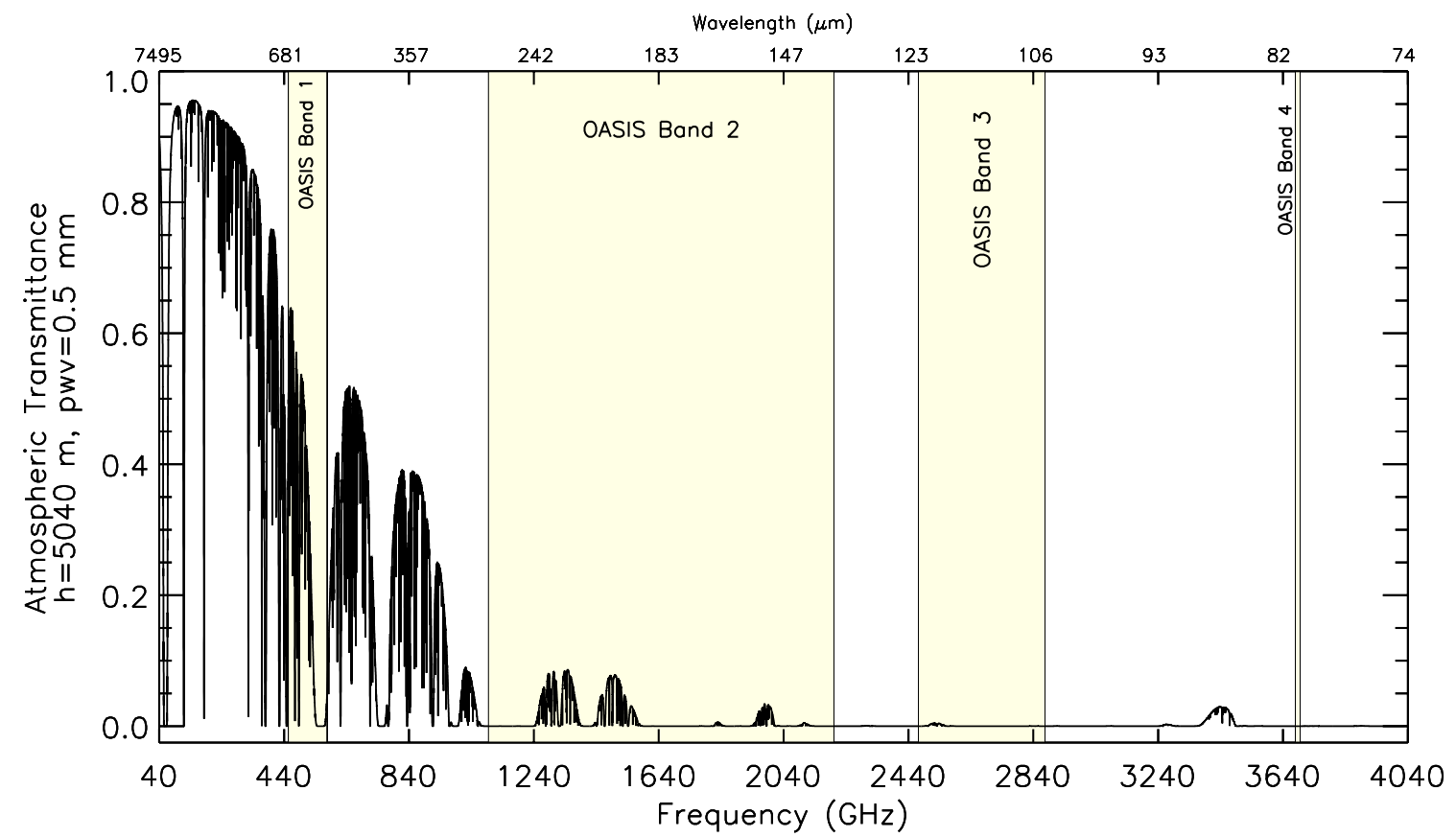

Figure 2. OASIS is optimized to operate at far-IR and submillimeter frequencies that are or nearly opaque to ground-based telescopes. Simulated terrestrial atmospheric transmission function (solid black curve), corresponding to the altitude of ALMA $(5040 \mathrm{~m})$ and a relatively low precipitable water vapor ( $\mathrm{pwv}=0.5 \mathrm{~mm}$ ), generated using the Planetary Spectrum Generator [6]. The OASIS Bands 1, 2, 3, and 4 (yellow rectangles) target wavelengths that are highly complementary to capabilities of ALMA and JWST.

\subsection{OASIS Observing Strategies}

In terahertz astronomy observing strategies have been developed with the goal of efficiently removing instrumental effects from the data, thereby providing an untarnished view of the target object. These strategies include position-switching (PS), 
dual-beam switching (DBS), frequency-switching (FS), load-chopping (LC), and on-the-fly mapping (OTF) [7,8]. All these techniques involve observing a line of sight (LOS) to a source at a frequency of interest and then observing a nearby LOS (or frequency) free of source emission. OASIS, like Herschel, is designed to support all these observing modes. Which mode an observer selects will depend on the extent of the source with respect to the OASIS beam and instrument stability. The less stable the instrument, the closer in time the observation of the reference position or frequency must be. The stability of a heterodyne receiver is often quantified in terms of its Allan time. The Allan time is the time during which an incoming signal can be integrated before the noise integrates down two standard deviations is slower than expected from the radiometer equation [8]. Beyond this point the observing efficiency significantly decreases, or may even go negative. In the case of the SIS and HEB receivers being flown on OASIS, the Allan time is expected to be between 20 and 30 seconds.

Approximately $70 \%$ of the $O A S I S$ observing time will be spent observing spatially unresolved protoplanetary disks. The target is acquired by first using the spacecraft star camera and reaction wheels to point the telescope within a few arcminutes of the target position. Fine pointing is accomplished by performing a spiral scan until continuum and/or spectral line emission from the target is detected by the science instrument. Once a target is successfullly acquired, a standard OASIS PS observing sequence will commence:

1) Integrate on target for a period of time less than the receiver's Allan time.

2) Use the focal plane scan mirror to move off target by several beamwidths and integrate for a predetermined time.

3) Use the scan mirror to move the receiver's beam back on target.

4) Use the flip mirror shown in Figure 3 to observe the calibration load for a few seconds.

5) While the heterodyne receivers are looking at the calibration load, the focal plane bolometer will be used to verify the telescope is still on target by monitoring the continuum emission from the source.

Beam from Corrector

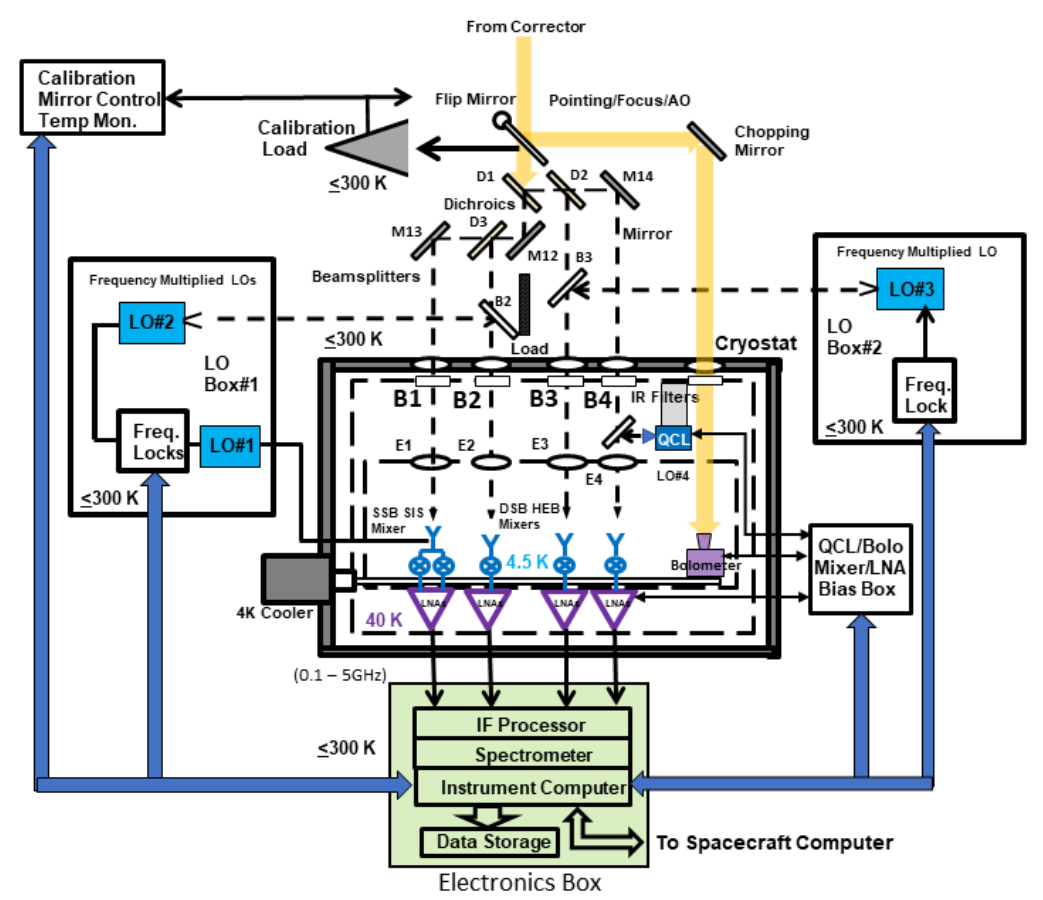

Figure 3: OASIS Instrument Block Diagram. OASIS uses cryogenic, low-noise Superconductor-Insulator-Superconductor (SIS) and Hot Electron Bolometer (HEB) mixers and solid-state local oscillators to downconvert incoming signals to frequencies where they can be efficiently amplified and conveyed to back-end spectrometers. The optical system employs dichroics that permit simultaneous observations to be performed in all four bands. 
This sequence will be repeated until the desired signal-to-noise ratio is obtained and can be modified as needed to support other observing modes. Since simultaneous observations occur in all four receiver bands, the total observing time required per object is set by the strength of the weakest line to be observed. The expected observing times per target are expected to be on the order of a few minutes for relatively bright planetary objects and up to $\sim 12$ hours for weak line sources (e.g., protoplanetary disks and external galaxies). The expected $5 \sigma$ sensitivities of OASIS in units of Jy/Beam $/ \mathrm{km} / \mathrm{s}$ as a function of frequency and integration time are provided in Figure 4. These sensitivities can be used to estimate the number of targets that can be observed during the mission.

Table 2. OASIS Performance Characteristics. The OASIS receivers have high sensitivity and wide spectral tunability in Bands 1, 2, and 3. Band 3 encompasses the HD (1-0) line at $112 \mu \mathrm{m}$ and Band 4 includes a para- $\mathrm{H}_{2} \mathrm{O}$ transition at $81 \mu \mathrm{m}$. All four bands are observed simultaneously. Spectrometer characteristics pertain to the Autocorrelation Spectrometer in all 4 bands. In addition, the properties of the Chip Transform Spectrometer are specified for Band 1. The spectral resolutions obtainable with these two spectrometers are typical values given nominal clocking rates.

\begin{tabular}{|c|c|c|c|c|}
\hline \multicolumn{5}{|l|}{ Facility } \\
\hline \multicolumn{5}{|l|}{ Antenna Diameter } \\
\hline Orbit & \multicolumn{4}{|l|}{ L1 Halo Orbit } \\
\hline Baseline Mission & \multicolumn{4}{|l|}{1 year } \\
\hline \multicolumn{5}{|l|}{ Extended Mission } \\
\hline \multicolumn{5}{|l|}{ Receivers } \\
\hline Frequency Range (GHz) & $\begin{array}{l}455 \\
575 \\
\end{array}$ & $\begin{array}{l}1100 \\
2200 \\
\end{array}$ & $\begin{array}{l}2475 \\
2875 \\
\end{array}$ & $\begin{array}{l}3682 \\
3692 \\
\end{array}$ \\
\hline Wavelength Range $(\mu \mathrm{m})$ & $\begin{array}{l}658.88 \\
521.38\end{array}$ & $\begin{array}{l}272.54 \\
136.27\end{array}$ & $\begin{array}{l}121.13 \\
104.28\end{array}$ & $\begin{array}{l}81.42 \\
81.20 \\
\end{array}$ \\
\hline System Noise (K) & 293 & 674 & 774 & 859 \\
\hline Polarizations & Dual & Single & Single & Single \\
\hline Mixer System & SSB & DSB & DSB & DSB \\
\hline Diffraction-limited Beam Size (arc-sec) & 8.5 & 2.7 & 1.6 & 1.2 \\
\hline Spectrometer & Band 1* & Band 2 & Band 3 & Band 4 \\
\hline Maximum IF Bandwidth (GHz) & $4 / 1$ & 3.5 & 3.5 & 3.5 \\
\hline Spectral Resolution (MHz) & $5.37 / 0.1$ & 5.37 & 5.37 & 5.37 \\
\hline Spectral Resolving Power & $10^{5} / 10^{7}$ & $3 \times 10^{5}$ & $5 \times 10^{5}$ & $1 \times 10^{6}$ \\
\hline Velocity Resolution $(\mathrm{km} / \mathrm{s})$ & $3.1 / 0.03$ & 1.0 & 0.6 & 0.4 \\
\hline Representative Lines & Band 1 & Band 2 & Band 3 & Band 4 \\
\hline Transition & $\mathrm{o}-\mathrm{H}_{2} \mathrm{O}\left(1_{10}-1_{01}\right)$ & $\mathrm{o}-\mathrm{H}_{2} \mathrm{O}\left(2_{12}-1_{01}\right)$ & $\mathrm{HD}(1-0)$ & $\mathrm{p}-\mathrm{H}_{2} \mathrm{O}\left(7_{26}-7_{17}\right)$ \\
\hline Frequency $(\mathrm{GHz})$ & 556.94 & 1669.90 & 2674.99 & 3691.32 \\
\hline
\end{tabular}




\section{OASIS SCIENCE OBJECTIVES}

OASIS will use its unique capabilities to achieve its overarching mission theme, which is to follow the cosmic water trail from galaxies, through protostellar systems, to solar system objects. This requires the following Science Objectives to be met:

1) Objective 1: Characterize the role of water in galaxy evolution and its part of the oxygen budget.

2) Objective 2: Characterize the time development of the water distribution and the role water plays in the process of planetary system formation.

3) Objective 3: Characterize the delivery of water to the solar system by investigating its known water reservoirs.

These science objectives resonate with the Astrophysics Roadmap and the 2010 Astrophysics Decadal Survey detailed in subsections 2.1,2.2, and 2.3.

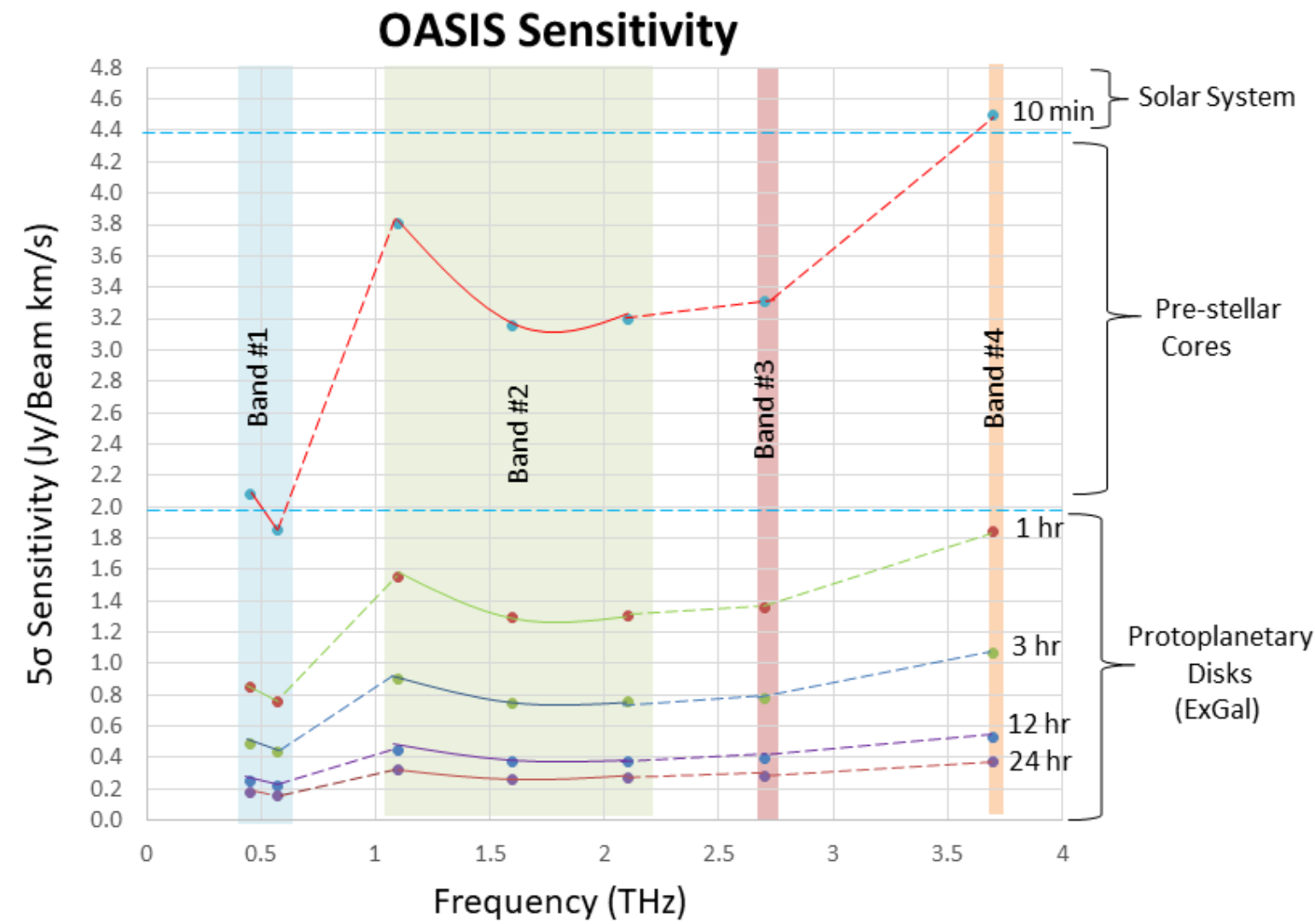

Figure 4. OASIS sensitivity as a function of frequency and integration time. All four bands are observed simultaneously. The receivers are continuously tunable over the frequency range of each band. Solar system objects are relatively close and bright, requiring integration times on the order of seconds or minutes. Many spectral lines will be detectable toward nearby, prestellar/molecular cores in less than an hour. Protoplanetary disks and extragalactic objects are expected to require several hours of integration to achieve desired signal-to-noise ratios. 


\subsection{OASIS Objective 1: Following the path of water to unveil the hidden growth of galaxies}

Star formation and Active Galactic Nuclei (AGN = accreting supermassive black holes, SMBHs) are fundamental processes that drive the evolution and build-up of galaxies. A considerable fraction of that build-up is deeply dustembedded since the bulk of the star formation takes place in dense interstellar clouds, and SMBHs go through their most vigorous growth phase while enshrouded in dust and gas. Obscured AGN span the full range of environments and astrophysical processes that drive the growth of SMBHs, and help to complete the picture of connections between the host galaxy and its black hole. Probing inside the thick layers is an observational challenge, since the dust obscures the regions from view at optical and ultraviolet (UV) wavelengths. These regions are therefore a largely unexplored phase of the growth of black holes and star formation. Studying the hidden, embedded phase of galaxy evolution is therefore key to many unsolved questions on how galaxies grow together with their SMBHs (co-evolution), how star formation progresses in extreme dense environments, and the nature of the main processes that govern black hole growth.

In the dense interstellar clouds, atoms come together to form molecules making them essential tracers of the physical, chemical and kinematic properties of the obscuring gas. Water forms from the oxygen that is released from exploding massive stars, and the hydrogen that originated in the Big Bang at the birth of the Universe. Water can reach extremely high abundances in dense gas where it serves as a unique signpost of the evolution of the embedded activity. Water that has formed as ice on the surface of dust grains in dense, cold, cores of molecular clouds can be sublimated into water vapour by energetic processes associated with star and planet formation, or with growing black holes. Water vapour has a rich spectrum with transitions mainly in the submillimeter and far-IR wavelength regime. At these wavelengths we can penetrate behind the veil of dust to explore the properties of the hidden star formation and growing black holes. Water emission in local, and intermediate redshift, galaxies is unobservable from the ground, but can be studied with OASIS at unparalleled sensitivity and resolution. $\mathrm{H}_{2} \mathrm{O}$ also has an exclusive feature of tracing the far-infrared radiation field, where the obscured activity has its peak energy output.

OASIS will follow the path of water in the evolution of galaxies: from the extremely water-rich dusty cores of powerful luminous and ultraluminous infrared galaxies (U/LIRGs) to the detailed study of the embedded accretion processes in nearby, more modest and numerous, star forming galaxies and AGN. The U/LIRGs are compact and well suited for the high resolution of OASIS. With a suite of water, its isotope and ionic lines, we can chart the origin and fate of the dusty nuclei, and unveil the nature of the buried luminosity sources in a complete sample ( $>100$ objects) of the local U/LIRGs. OASIS will also close an important knowledge gap between the luminous distant, high-redshift $(\mathrm{z}>3)$ galaxies, and the local and intermediate (out to $\mathrm{z}=0.8$ ) redshift $\mathrm{U} /$ LIRGs. This epoch is a missing link in understanding the slowing down of the evolutionary processes. Were water rich nuclei more common in dusty galaxies in the younger Universe and what is their role in the rapid, evolution of the SMBHs? OASIS also has the unique capacity to detect fine-structure lines of oxygen, nitrogen and carbon in intermediate redshift galaxies. These lines are key to probing the evolution of nucleosynthesis and astration, and together with the vital water molecule, they complete the picture of energetic dustenshrouded accretion.

Water is an essential part of the oxygen budget of interstellar clouds and OASIS will explore how the role of water changes with environment and metallicity (proportion of elements heavier than helium). OASIS' exceptional spectral and spatial resolution allow us to follow the path of water through a galaxy: how it transitions from the extended gas in the galaxy disk and into the star- and planet-forming clouds. In nearby ( $<10 \mathrm{Mpc}$ ) galaxies, OASIS can resolve structures on scales of molecular clouds and the unique high velocity resolution of OASIS enables the separation of features on even smaller scales. A large number of nearby galaxies offers a variety of environments where OASIS will study crucial aspects of relations between the properties of interstellar gas and the rate at which that gas forms stars. OASIS will also investigate how SMBHs in nearby (less powerful) AGN grow together with the obscuring gas.

With $O A S I S$ we have the chance to detect water in local low-metallicity galaxies, where water abundances are also expected to be interestingly high. Tracing the path of water into metal poor environments is fundamental to our understanding of galaxy assembly, and the conditions of star and planet formation in the early universe. "Metallicity" is a concept used by astronomers to describe enrichment of the interstellar medium (ISM) by the release of processed material from dying stars. The more stellar generations a galaxy has gone through, the more "metals" are found mixed up with the primordial hydrogen. Regions of low metallicity are expected to be more common in the early days of the Universe, but regions of unenriched environments also exist today, often in local dwarf galaxies that provide an opportunity to explore conditions akin to those in the early Universe at high resolution and sensitivity. Again, the combination of a large collecting area and high spectral resolution lets $O A S I S$ detect water in these extreme and important environments for the first time. OASIS will also observe deuterated molecular hydrogen, HD, to find hitherto undetected, "dark" molecular gas, which is 
likely the main molecular phase in metal poor galaxies, but also shows surprising abundance in regions of more normal, Solar-like metallicity. The dark molecular gas is undetectable with standard molecular tracer molecules (such as CO) but can be detected by the faint HD line. The large collecting area of OASIS equips it uniquely to map HD emission in nearby galaxies for the first time.

The OASIS extragalactic path of water will address fundamental, high-priority science questions that stem from the NASA Astrophysics Roadmap [Enduring Quests Daring Visions: NASA Astrophysics in the Next Three Decades]: For example, goal 3.3 of the Roadmap asks, How did we get here - The history of galaxies. Dust obscured star formation and black hole growth is fundamental to the evolution of galaxies, and water is a key molecule for this, in particular in the final, most extreme stages of accretion, where OASIS will provide crucial information. Metal-poor galaxies are also essential ingredients in the history of galaxies, and OASIS has the capacity to provide absolutely unique information on the role of water in these unusual environments. The history of galaxies is also governed by processes that transform diffuse gas into star forming clouds, where $O A S I S$ will make groundbreaking contributions through its outstanding spatial- and velocity resolution. In addition, the $O A S I S$ extragalactic mission addresses major goals under the heading "Understanding the Cosmic Order" in the current Astrophysics Decadal Survey [New Worlds, New Horizons in Astronomy and Astrophysics in the decade 2012-2021]. In particular, the survey cites the following Science frontier questions: How do baryons cycle in and out of galaxies, and what do they do while they are there? What controls the mass-energy-chemical cycles within galaxies? How do black holes grow, radiate, and influence their surroundings? Again, these questions address fundamental queries on the growth and history of galaxies, where OASIS will make unique contributions. OASIS will observe and explore the rich and varied water emission in dusty, luminous as well as metal poor galaxies to get the complete picture of the galactic trail of water from the extended gas in galaxies and into the birth of stars and the growth of black holes.

The following subsections expand on the cases for conducting cutting-edge extragalactic science following the water trail with $O A S I S$

\subsubsection{Water as a probe of hidden galaxy nuclei}

The peak of galaxy growth (the so-called Cosmic Noon) occurred already at a redshift of $z=1-3$, but rapid evolution still continues in the local, dusty luminous and ultraluminous infrared galaxies (LIRGs and U/LIRGs with $\mathrm{L}_{\mathrm{IR}} \sim \mathrm{L}_{\text {bol }}=10^{11}$ $10^{12} \mathrm{~L}_{\text {sun }}$ ). They are mainly powered by extreme bursts of star formation and/or AGN. LIRGs and U/LIRGs are often the result of mergers or interactions between gas-rich galaxies where enormous amounts of interstellar matter (ISM) are funneled into the centers. LIRGs and U/LIRGs are essential to our understanding of how galaxies evolve and they contribute a fundamental part of the whole galaxy build-up in the Universe $[9,10]$. The infrared emission of U/LIRGs stems from emission absorbed and re-emitted by dust enshrouding the extraordinary starburst/AGN activity that provides most of the luminosity. In some cases the nuclei are extremely obscured by dust, announcing the presence of dense gas that holds a key to the understanding of nuclear growth and feedback mechanisms. Understanding the molecular properties of U/LIRGS is essential both for defining the evolution of present-day galaxies and addressing crucial astrophysical processes in their even more energetic intermediate redshift cousins. 

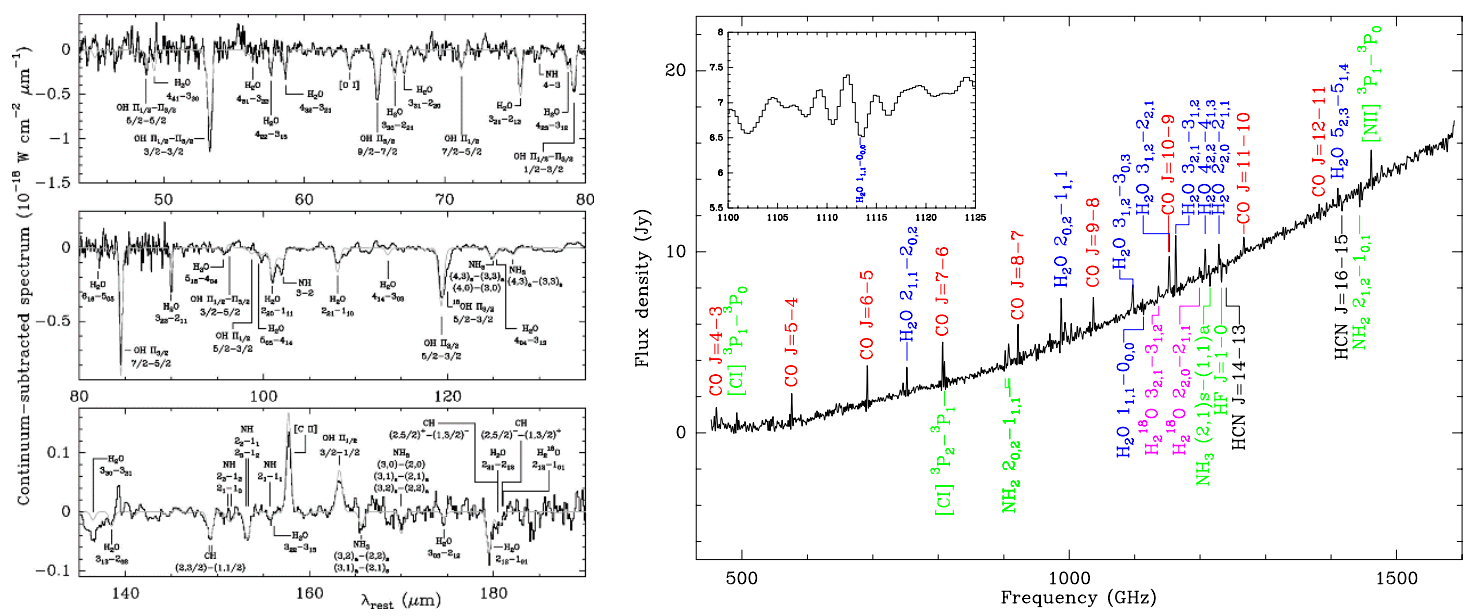

Figure 5. Left: Continuum-subtracted Infrared Space Observatory (ISO) spectrum of Arp 220 showing a rich spectrum including prominent $\mathrm{H}_{2} \mathrm{O}$ lines (reprinted from [11]). Right: Herschel SPIRE spectrum of the water-rich LIRG Zw049.057 revealing luminous, but unresolved, water emission (in blue)(reprinted from [12]). OASIS will expand the current sample of U/LIRGs by more than an order of magnitude to fully chart the trail of water in dust embedded nuclei at unprecedented spatial and spectral resolution

Water emission can only be detected from space, and the Herschel Space Observatory broke new ground in detecting $\mathrm{H}_{2} \mathrm{O}$ in a number of local luminous galaxies $[12,13,14,15,16,17,18,19,20,21]$ following early studies with the ISO satellite [11, 22] (Figure 5). The $\mathrm{H}_{2} \mathrm{O}$ line emission is luminous (Figure 5), and abundances can become high in the inner regions of U/LIRGs $[12,15]$. The water abundances can reach $X\left(\mathrm{H}_{2} \mathrm{O}\right)=10^{-5}$ (with respect to $\mathrm{H}_{2}$ ) on scales of up to 100 pc. This water abundance is normally seen only on sub-parsec scales in Galactic, star forming hot cores ${ }^{1}$. There appears to be a strong link between $\mathrm{H}_{2} \mathrm{O}$ and the far-IR radiation field in LIRGs and U/LIRGs [23] (Figure 6). $\mathrm{H}_{2} \mathrm{O}$ can couple strongly to the radiation field in warm regions with intense far-IR emission [24]. The radiative excitation depends on the far-IR radiation density, which is related to the compactness of the far-IR source. $\mathrm{H}_{2} \mathrm{O}$ is therefore an excellent, tracer of dusty galaxy nuclei, their far-IR radiation fields and hidden, embedded luminous sources [12, 21, 23, 24]. This sets $\mathrm{H}_{2} \mathrm{O}$ aside from more commonly used extragalactic tracers of molecular gas, such as CO. However, only a few U/LIRGs in the local universe have had their $\mathrm{H}_{2} \mathrm{O}$ properties studied in detail, and even fewer galaxies were observed at high spectral resolution with the Herschel HIFI high resolution spectrometer [20,21]. OASIS will expand the current sample of U/LIRGs by an order of magnitude to fully chart the trail of water in dust embedded nuclei.

Highly excited lines of $\mathrm{H}_{2} \mathrm{O}$ (e.g., at $\lambda=248.2,212.5$, and $71.9 \mu \mathrm{m}$ ) have proven efficient in identifying compact obscured nuclei [12, 15, 16, 24] and enshrouded AGN [21]. $\mathrm{H}_{2} \mathrm{O}$ modeling by Gonzalez-Alfonso et al. [24] of $\mathrm{H}_{2} \mathrm{O}$ show that the highest-lying $\mathrm{H}_{2} \mathrm{O}$ lines with energies in excess of $400 \mathrm{~K}$, require large $\mathrm{H}_{2} \mathrm{O}$ columns and are formed in warm regions with dust temperatures above $90 \mathrm{~K}$. Combining lower and higher excitation line ratios, and the simultaneous observation of the continuum, give the strength of the far-infrared radiation field, the relative impact of collisional excitation for the lowerenergy transitions and the water column density. High abundances of $\mathrm{H}_{2} \mathrm{O}$ can have different origins: they can for example stem from ion-neutral reactions linked to cosmic ray or X-ray ionization of $\mathrm{H}_{2}$ [25]. In warm $(\mathrm{T}>250 \mathrm{~K})$ gas, neutralneutral reactions that produce $\mathrm{H}_{2} \mathrm{O}$ are also possible [25]. The dense gas clouds also contain small 0.001-0.1 $\mu \mathrm{m}$ sized dust grains - solid particles that consist of amorphous silicates and carbonaceous material. These particles are important partially because they constitute surfaces on which water can form. If the dust temperature increases above a $T_{d}$ of $100 \mathrm{~K}$, $\mathrm{H}_{2} \mathrm{O}$ will sublimate from its mantle of ice on the dust particle, into the gas phase where it can reach very high abundances $[25,26]$. With OASIS we have unique access to a suite of water lines at high spectral and spatial resolution allowing us to carry out a complete census of water in the LIRGs and U/LIRGs. We will have the full picture of how water formation processes and excitation link to the host galaxy properties, its interaction and merger status, and to the evolution of dusty nuclear activity.

\footnotetext{
${ }^{1}$ The extremely water-rich dusty nuclei of U/LIRGs and LIRGs have counterparts of similar regions in the Milky Way: the compact hot cores. These are regions of large dust and gas column density - just like the obscured galaxy nuclei. Conditions are very similar, but the Galactic hot cores are significantly smaller with linear sizes of only 0.01-0.03 pc. The Galactic compact hot cores are also important targets for $O A S I S$ taking advantage of the high spatial and spectral resolution.
} 
The water lines will consist of a combination of multiple lines of ortho- and para-water, which is important in addressing formation, conditions and the thermal history of the observed water. At high temperatures $(\mathrm{T}>50 \mathrm{~K})$ the ortho-to-para ratio reaches a limit of three, while in cold formation processes, the ratio approaches zero [25]. Furthermore, simultaneously with the main water observations, OASIS will observe lines of water ions, such as $\mathrm{H}_{3} \mathrm{O}^{+}$and $\mathrm{H}_{2} \mathrm{O}^{+}$as well as lines of $\mathrm{OH}$. These lines are the main gas-phase precursors of water, leading to a complete account of the chemical network and formation of water [25]. Different natures and evolutionary stages of the buried activity will have different paths of water formation and destruction - making its history a key diagnostic tool for the galaxy's nuclear evolution. In addition, water ions and $\mathrm{OH}$ will provide ionization rates, which will help determine the nature of the enshrouded sources.

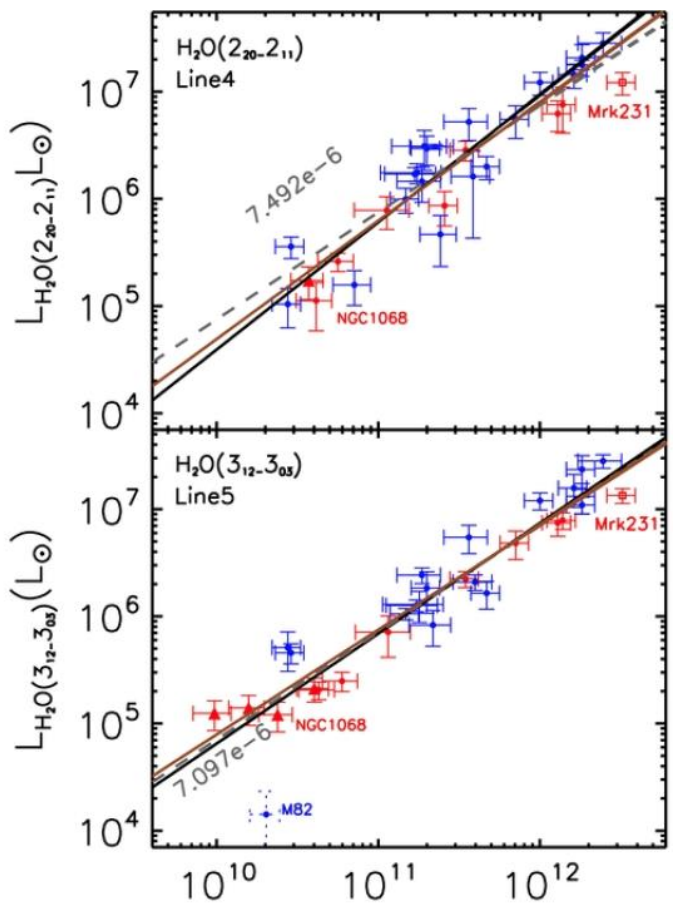

Figure 6. Figure showing the line intensities of para- $\mathrm{H}_{2} \mathrm{O} 220-211$ and ortho- $\mathrm{H}_{2} \mathrm{O} 3_{12}-3_{03}$ lines (y-axis) plotted against the galactic IR luminosity $\mathrm{L}_{\mathrm{IR}}$ in solar units on the $\mathrm{x}$-axis (reprinted from [24]). $\mathrm{L}_{\mathrm{H} 2 \mathrm{O}}$ is found to correlate to $\mathrm{L}_{\mathrm{IR}}$ as $\log \mathrm{L}_{2 \mathrm{O}}=\alpha \log \mathrm{LIR}+\beta$, where a ranges between 0.8 and 1.2 depending on the transition.

Herschel took a significant step forward in detecting extragalactic water, but only a handful of galaxies have their line profiles resolved with the high-resolution HIFI instrument [19, 20, 21]. The SPIRE spectrometer did not spectroscopically resolve the $\mathrm{H}_{2} \mathrm{O}$ lines at all, providing only a tantalizing spike of water (Figure 5). The higher frequency PACS instrument did somewhat better showing a hint of interesting structure in the luminous water lines (Figure 7). Feedback processes in U/LIRGs and LIRGS in the form of winds or outflows have primarily been detected in the far-infrared $119 \mu \mathrm{m} \mathrm{OH}$ transition [27], but in some cases Herschel also found $\mathrm{H}_{2} \mathrm{O}$ lines in the form of tentative P-Cygni or reversed P-Cygni shapes - suggesting that water-rich gas may be either outflowing, or inflowing to the center $[16,20]$. OASIS will resolve the line shapes in all luminous dusty galaxies, to determine the role of water in the dynamical processes of the embedded activity, and to reveal how excitation conditions and abundance are changing with location in the nucleus. 


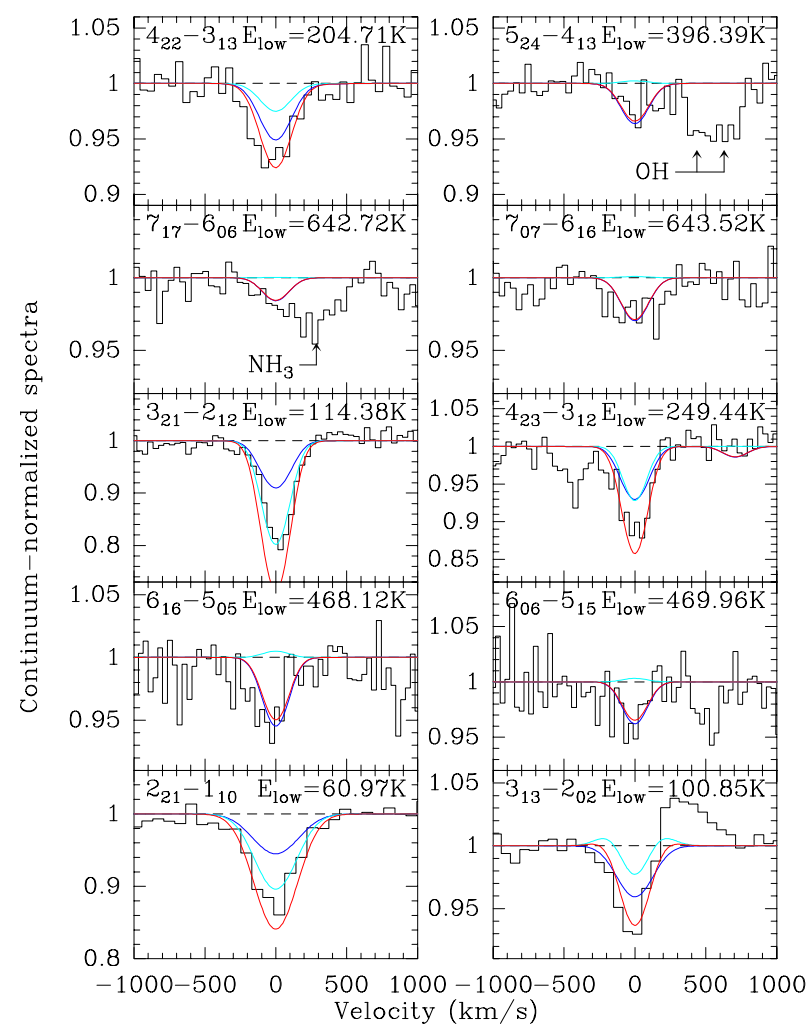

Figure 7. Sample Herschel/PACS $\mathrm{H}_{2} \mathrm{O}$ spectra of the LIRG galaxy Zw049.057 (reprinted from [12]). Tentative P-Cygni profiles may be there in the lower-energy line, but the lines are not really resolved even with PACS. The colored lines represent different model fits. OASIS will resolve the line shapes in all luminous dusty galaxies, to determine the role of water in the dynamical processes of the embedded activity, and to reveal how excitation and abundance is changing with location in the nucleus.

\subsubsection{Water isotope observations with $O A S I S$ will trace the properties of star formation}

One of the great challenges with the embedded, dust-enshrouded galaxy nuclei is to trace star formation and how the massive stars are chemically enriching their dusty surroundings. Because of the dust absorption, the regions are unreachable by standard UV or optical methods to track the evolution of the elements. A major issue is also how the initial mass function (IMF) - the mass distribution of newly-formed stars - changes in the dense nuclear environment. The IMF describes the distribution between low- and high-mass stars that are born in a molecular cloud. Normal star formation often follows a so-called Salpeter IMF [28] where low mass stars dominate the total mass in the star formation process. It is crucial to know the IMF in order to understand the mass-energy-chemical cycles, how the host galaxies are evolving together with their black holes, and how the star formation processes are changing with environment.

The ${ }^{16} \mathrm{O}$ and ${ }^{18} \mathrm{O}$ isotopolog-ratios are proposed to be key tools in investigating the IMF. ${ }^{18} \mathrm{O}$ is a secondary nuclide, produced by He burning in massive stars and may enrich the ISM through stellar winds or supernova ejecta [29]. Models suggest that an elevated ${ }^{18} \mathrm{O} /{ }^{16} \mathrm{O}$ ratio is the result of a "top-heavy" IMF (skewed towards more massive stars) [30] as well as serves as an indicator of the age of a starburst. More studies are required to establish the line ratio as an IMF tracer. OASIS will obtain sensitive, high-resolution lines of the ${ }^{18} \mathrm{O}$ isotopic variant of both $\mathrm{H}_{2} \mathrm{O}$ and $\mathrm{OH}$, to map the time evolution of the IMF and nucleosynthesis in obscured galaxy centres and connect them to the interaction state and the properties of the host galaxy. The advantage of using the water isotopes is that they target the most embedded regions, which are the hardest to reach with standard methods, and are also the locations of the most active evolution. In luminous, dusty galaxy nuclei there is evidence of elevated ${ }^{18} \mathrm{O}[12,15]$ and there are indications that a disproportionate number of massive stars are born [31]. Initial studies with Herschel also point to a connection between the ${ }^{18} \mathrm{O} /{ }^{16} \mathrm{O}$ ratio and the presence of largescale inflows in LIRGs and U/LIRGs [16]. Such flows can be caused by galaxy-galaxy interactions,, but also by more secular evolutionary processes, such as gas flowing along a stellar bar. 


\subsubsection{OASIS will follow the path of water out to intermediate redshift U/LIRGs and LIRGs}

The high sensitivity of OASIS enables observations of $\mathrm{H}_{2} \mathrm{O}$ in luminous galaxies at intermediate redshifts out to $\mathrm{z}=0.8$. This is a not-well-studied part of the evolutionary history of the universe when the frenetic growth of galaxies taking place in the cosmic noon (at higher redshift $\mathrm{z}=1-3$ ) is winding down. The intermediate redshift universe therefore constitutes an important missing link between the high-redshift and our local Universe. At redshifts roughly ranging between $0.3<z<1.0$, the star-formation rates and gas fractions [32], are believed to be significantly higher than those in the local universe (this is sometimes referred to as part of the process of "downsizing"). As we move to higher redshifts, further back in time, galaxies with U/LIRG-like luminosities, begin to dominate the star formation activity in the Universe at about z 0.7 [33] (see also Figure 13, right panel).

OASIS' unrivalled collecting area and sensitivity make it the only facility that can access fundamental water lines in the intermediate redshift Universe, to probe the dusty nuclei of the luminous galaxies there. With OASIS we will measure $\mathrm{H}_{2} \mathrm{O}$ lines in a large, complete sample of intermediate redshift U/LIRGs. We selected water lines of crucial diagnostic value, for example: $\mathrm{o}-\mathrm{H}_{2} \mathrm{O} 5_{23}-5_{14}$, at a rest wavelength $\lambda_{\text {rest }}=212.5 \mu \mathrm{m} ; \mathrm{p}-\mathrm{H}_{2} \mathrm{O} 3_{13}-2_{02}(138.5 \mu \mathrm{m})$; and o- $\mathrm{H}_{2} \mathrm{O} 4_{23}-3_{12}(78.7 \mu \mathrm{m})$. The $\mathrm{H}_{2} \mathrm{O}$ observations will target the nature, evolutionary status and water formation processes of the nuclear embedded activity of the U/LIRGs. With higher gas fractions and star formation activity, we expect the water content and luminosity of intermediate redshift galaxies to be higher than what is found for local U/LIRGs. Intense $\mathrm{H}_{2} \mathrm{O}$ emission is found in luminous galaxies at high redshifts $>3$ (Figure 8 ) (accessible by ALMA) $[34,35,36,37]$ where it is for some cases proposed to be an efficient probe of embedded star formation. However, the lack of multi-transition information makes it unclear how different gas and metallicity distributions affect the water emission and its interpretation. Also unknown is how the transitional stage of the intermediate redshift galaxies affect their properties in comparison to luminous galaxies in the local and high-redshift Universe.

Evidence suggesting that the U/LIRG populations in the local and intermediate redshift Universe have disparate properties [38], raises questions, for example, on how the fraction of extremely obscured nuclei vary between local and intermediate redshift galaxies. OASIS will address key queries on whether the existence of large-scale (30-100 pc) regions of extremely high water abundance are as common at intermediate redshifts as in the present day Universe. How does nuclear obscuration evolve with luminosity and host galaxy properties (such as mass and state of interaction) and what is the impact of water-richness on the efficiency of star formation and on the growth of the SMBHs? OASIS will also carry out important complementary observations of fine structure lines of atomic oxygen, carbon and nitrogen charting the larger scale evolution of metals and star formation in the intermediate redshift galaxies (Section 2.1.7 below).
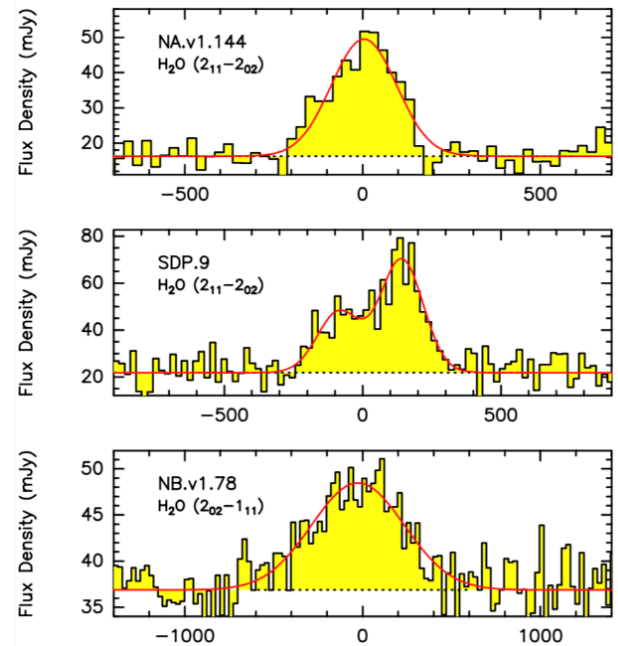

Figure 8. Spectra from the IRAM Plateau de Bure Interferometer (PdBI) showing intense water emission in galaxies at redshift $\mathrm{z}>3$ (reprinted from [35]). $\mathrm{H}_{2} \mathrm{O}$ lines are among the strongest molecular lines in high- $\mathrm{z}$ U/LIRGs. OASIS ' unrivalled collecting area and sensitivity makes it the only facility that can access fundamental water lines in the intermediate redshift Universe, out to $\mathrm{z}=0.8-1$. This is an important, but relatively unexplored, transition stage between the local Universe (at redshift 0$)$ and the vigorous growth in the Cosmic Noon $(\mathrm{z}=1-3)$. Observing this intermediate redshift range is key to understanding the notable change in the supermassive black hole growth and the Star Formation Rate Density (SFRD) that Universe undergoes at this time. 


\subsubsection{Water tracing the feeding and feedback in nearby starbursts and AGN}

Because they are relatively rare in the local Universe, U/LIRGs and LIRGs are mostly found at distances $>60 \mathrm{Mpc}$ and tend to be very compact and in many cases are extremely water rich on relatively large scales up $100 \mathrm{pc}$. Nearby (D < 15 Mpc) star forming galaxies and AGN do not host such extreme regions on matching linear scales, but instead offer an opportunity to follow the trail of water in varying galactic environments at an unprecedented spatial resolution on cloudscale level. Lower luminosity starbursts are more numerous than U/LIRGs and constitute a larger fraction of the local star formation density. Studying them is essential for our understanding of normal, disk-dominated star formation. The strength of OASIS is its exceptional collecting area and spectral resolution, which makes it an ideal instrument to study the cycle of water, its ions, and isotopic variants in nearby starburst galaxies and AGN..

The study of water in dense star forming clouds and how these clouds connect to the surrounding diffuse molecular gas component provides essential information on the star formation processes and the evolution and delivery of water into dense molecular cloud cores and ultimately into protoplanetary systems. Water is ubiquitous also in the diffuse molecular medium [19] and through combining $\mathrm{H}_{2} \mathrm{O}$ lines with $\mathrm{OH}$ and water ions $\left(\mathrm{H}_{2} \mathrm{O}^{+}\right.$and $\left.\mathrm{H}_{3} \mathrm{O}^{+}\right)$we can trace how the water and oxygen chemistry change with environment, from the extended gas and into the dense star forming gas. Combining measurements of high- and low-excitation lines is vital in order to account for the varying conditions from the diffuse gas and the star forming clouds (Figure 9). Changing ionization rates will be mapped with water and $\mathrm{OH}$ ions in unprecedented detail. Previous studies suggest steep gradients (by a factor of 10) in ionization rates from galaxy centres out into their disks. How and where the transition occurs is however not understood with current, low-resolution studies.

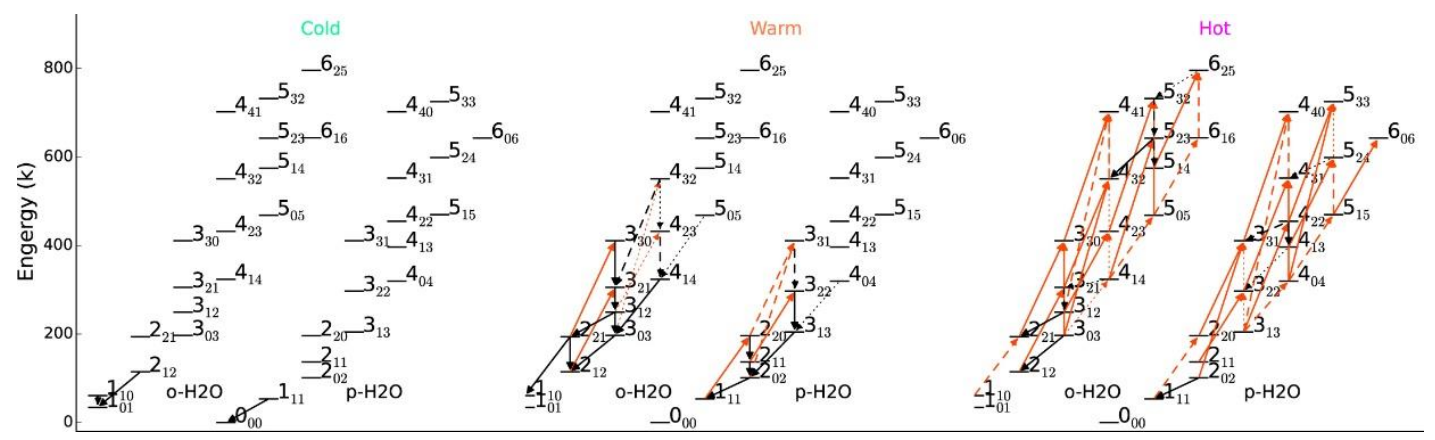

Figure 9. Showing models by Liu et al. [20] of the distributions of water level populations of multiple ISM components denoted cold, warm and hot. The black downward-pointing and red upward-pointing arrows indicate emissions and absorptions, respectively. (Figure reprinted from [20]). OASIS will trace how the water and oxygen chemistry change with environment, from extended diffuse regions and into the dense star forming gas, from cool environments and into hot, active regions.

Dynamical processes within the star forming regions, and in the diffuse gas, occur on sub-pc scales, and to resolve them spectrally requires velocity resolutions better than $1 \mathrm{kms}^{-1}$. Both star forming clouds and the extended gas, between the clouds, are subject to forming filamentary structures — scales ranging from $0.2 \mathrm{pc}$ to $>100 \mathrm{pc}$ in the extended gas $[39,40]$. Such filaments (or "spurs," or "feathers") have been discovered in the Milky Way and may connect local and large-scale processes of star formation in galaxies. Feedback from the newly born stars will also directly impact the velocity structure, excitation and abundance of water in the star forming clouds.

Galaxy-wide processes (such as spiral arms and bars) impact cloud properties, water content and star formation. For example, in the nearby, prototypical starburst galaxy M82, water abundances and dynamics seem linked to the larger scale dynamical processes of gas motions in the stellar bar potential [19]. The line profiles of water change depending on which water line we observe, which position in the galaxy we point at and also compared to other species (Figure 10). OASIS will study the oxygen isotopic ratios at high resolution and in varying environments to investigate if the stellar IMF is changing with environment in similar ways as in U/LIRGs. 
OASIS will increase the spatial resolution of existing extragalactic water observations by a factor of five and will follow the trail of water in a large number of nearby galaxies with varying properties. We will have a complete map of how largeand small-scale processes impact the trail of water in galaxies and the necessary velocity and spatial resolution to disentangle the contributions of the various ISM structures along the line of sight. This way OASIS will address crucial aspects of relations between the properties of interstellar gas and the rate at which that gas forms stars.
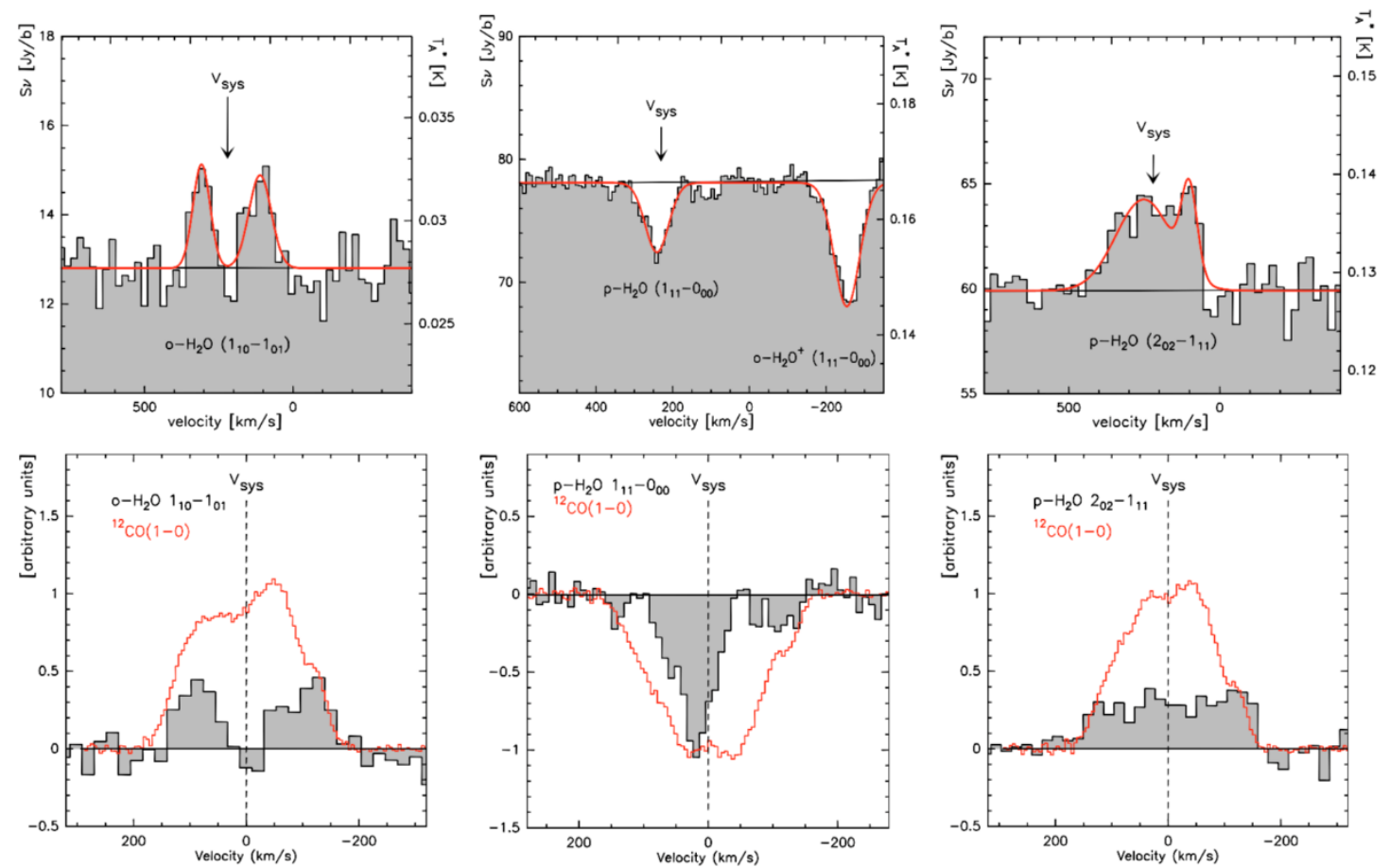

Figure 10. Herschel HIFI spectroscopy of low-level water transitions in the starburst galaxy M82 (reprinted from [19]). The top panels show lines of ortho- and para- $\mathrm{H}_{2} \mathrm{O}$ as well as ortho- $\mathrm{H}_{2} \mathrm{O}^{+}$. The lower panels show comparisons with $\mathrm{CO}$ profiles (red) at matching resolution. This varying line profiles illustrates the requirement for sufficient spectral resolution of the line profiles. OASIS will increase the spatial resolution of existing extragalactic water observations by a factor of ten and will follow the trail of water in a large number of nearby galaxies with varying properties. We will have a complete map of how large- and small-scale processes impact the trail of water in galaxies and the necessary velocity and spatial resolution to disentangle the contributions of the various ISM structures along the line of sight.

Herschel has found water in nearby AGN, for example in the iconic Seyfert galaxy NGC1068 [41]. The $\mathrm{H}_{2} \mathrm{O}$ emission is unresolved in the Herschel beam, suggesting it originates mostly in the circumnuclear disk (CND) around the AGN rather than in the starburst ring. Interestingly, comparing the $\mathrm{H}_{2} \mathrm{O}$ properties of $\mathrm{NGC} 1068$ with that of the more powerful and luminous U/LIRG AGN, Mrk231 [14], reveals significant differences in the water excitation between the two sources. For both galaxies the $\mathrm{H}_{2} \mathrm{O}$ emission is concentrated near the black hole, but the water in Mrk231 is significantly more excited. Water excitation and abundances (together with those of the water ions) are excellent tools in probing the evolution of the torus around the AGN, and measure the power and impact of the AGN.

OASIS can resolve the water emission of nearby AGN such as NGC1068, pinpoint its location to the CND and accurately determine excitation, water abundances and kinematics, for example when searching for signs of feedback in the line shapes. The role of water in the dense dust tori surrounding growing SMBHs in AGN can be studied by OASIS in unrivalled detail. With OASIS we will study the CND regions of a complete sample of nearby AGN of varying accretion state, mass and obscuration, to fully chart the impact of the AGN on the formation and destruction of water in the CND.

\subsubsection{OASIS will explore water in low-metallicity systems}

The first galaxies in the Universe were formed from a metal-free pristine interstellar medium, but after the first episode of star formation, they were rapidly enriched with metals from the explosions of the first supernovae ( $\mathrm{SNe}$ ) [42] These early 
SNe produced oxygen whose abundance dominated over other metals at early times, as shown in Figure 11. The dominance of oxygen over carbon means that, in terms of element availability, early galaxies are expected to be rich in water, $\mathrm{H}_{2} \mathrm{O}$.

Early galaxies were metal poor, with chemical properties akin to those in nearby low-metallicity dwarf galaxies. Dwarfs are the most abundant at any cosmic epoch, and considered to be the "building blocks" of more massive galaxies, under the currently-accepted paradigm for structure formation [43]. Thus, understanding the physical conditions in their ISM is key to testing hypotheses corresponding to different galaxy evolution scenarios. Low-metallicity dwarf galaxies in the Local Universe may not resemble early galaxies in all respects, but they are very similar from the standpoint of chemical evolution. Thus, $\mathrm{H}_{2} \mathrm{O}$ because of its expected high abundance at low metallicity, may be an important probe of physical processes of galaxy evolution, but so far little, if anything, is known about $\mathrm{H}_{2} \mathrm{O}$ in this regime, because $\mathrm{H}_{2} \mathrm{O}$ is not observable from the ground in nearby galaxies and Herschel lacked the sensitivity needed to detect them.

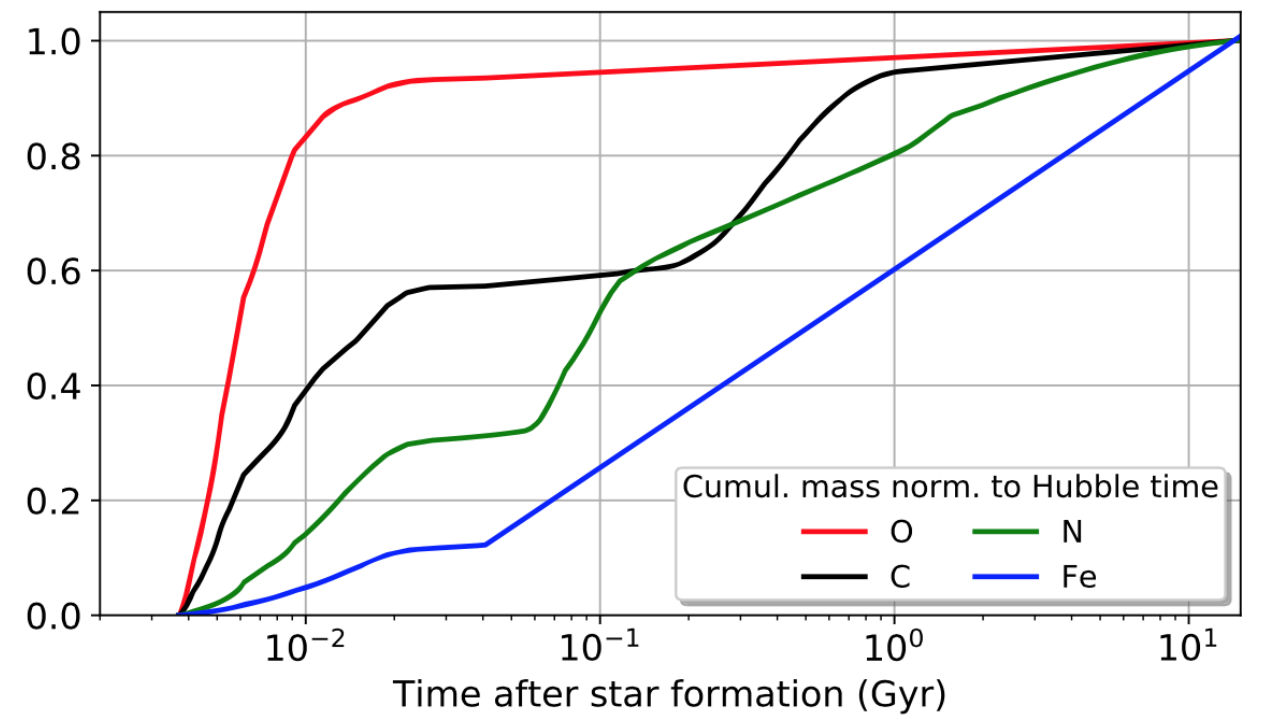

Figure 11. Timescales of production of various elements, in cumulative mass produced, after a single episode of star formation (a single stellar population) of solar metallicity, normalized to the amount present after one Hubble time (reprinted from [44]). The abundance patterns for primordial SNe are similar [45]. The most abundant element is oxygen (red line), which is mainly produced by Core-Collapse SNe and therefore has the shortest formation timescales. The least abundant element, iron (blue line) comes from type Ia SNe, while carbon (black), has contributions from both kinds of SNe and from AGB stars. Carbon needs approximately $1 \mathrm{Gyr}$ to achieve the same abundance as oxygen. With the exceptional capacity of OASIS we will, for the first time, detect $\mathrm{H}_{2} \mathrm{O}$ in nearby low-metallicity dwarf galaxies, enabling an unprecedented view of dwarf galaxy evolution and their star-forming processes

Interestingly, chemical models predict that in galaxies like the Magellanic Clouds (the two closest dwarf galaxies) $\mathrm{H}_{2} \mathrm{O}$ abundance is comparable to that of CO. For galaxies in the early Universe, because of the lack of available carbon (see Figure 11), $\mathrm{H}_{2} \mathrm{O}$ is expected to exceed $\mathrm{CO}$ [46]. This makes $\mathrm{H}_{2} \mathrm{O}$ a fundamental tracer of physical conditions in the starforming regions of metal-poor galaxies. The emission lines of dwarf galaxies are generally quite narrow (10-100 kms $\left.{ }^{-1}\right)$ and to detect them requires high spectral resolution and sensitivity. With the exceptional capacity of OASIS we will, for the first time, detect this vital molecule in nearby low-metallicity dwarf galaxies, enabling an unprecedented view of dwarf galaxy evolution and their star-forming processes. It will also be possible to link the properties of nearby dwarf galaxies determined by $O A S I S$ with those of galaxies in the early Universe whose $\mathrm{H}_{2} \mathrm{O}$ can be observed from the ground. Local dwarf galaxies are very numerous, with a large number of objects available for study.

\subsubsection{OASIS will use HD to probe CO-dark gas}

$\mathrm{CO}$ is the most abundant molecule after $\mathrm{H}_{2}$ (the $\mathrm{CO}$ fractional abundance with respect to $\mathrm{H}_{2}$ being $\sim 10^{-4}$ ) and can be used as a tracer of the molecular mass of galaxies, as it is much easier to observe than the $\mathrm{H}_{2}$ molecule with its low dipole moment. $\mathrm{CO}$ can be detected in relatively low density regions $\left(\mathrm{n}>100 \mathrm{~cm}^{-3}\right)$. Using the millimeter/submillimeter rotational transitions of $\mathrm{CO}$ as tracers of the $\mathrm{H}_{2}$ distribution and average physical conditions in galaxies is now a well-established 
method.. Most studies have focused on using the two lowest $\mathrm{CO}$ rotational lines, whose intensities can then be converted into $\mathrm{H}_{2}$ mass via the so-called $\mathrm{X}_{\mathrm{CO}}$-factor $[47,48,49,50]$. All these studies, however, rely on the premise that CO is coexistent with and in constant proportion to $\mathrm{H}_{2}$. In actuality, extensive observational and theoretical work has now shown that a CO-faint phase becomes possible for the ISM if the average metallicities are low [51] and/or if strong FUV fields are present $[52,53]$. Recent models have also shown that even high cosmic ray ionization rates, expected for example in starburst or AGN-dominated galaxies, can destroy $\mathrm{CO}$ (but not $\mathrm{H}_{2}$ ). This implies that even high-density solar-metallicity regions (where the FUV fields would not penetrate) could host large $\mathrm{H}_{2}$ gas reservoirs devoid of CO.

Various other tracers of this CO-dark gas have been proposed, for example lines of atomic carbon [C I] [39, 54]. However, while the [C I] line emission may extend well beyond that of low-J CO line emission, its detection would be restricted to relatively low density, high temperature regimes. In general, most of the $\mathrm{CO}$ alternatives proposed suffer from either being too low in abundance or, most importantly, not truly able to trace the extent of the $\mathrm{H}_{2}$ molecule and hence the molecular mass of a galaxy. An obvious contestant to overcome this limitation is $H D$, the heavy isotope of molecular hydrogen.
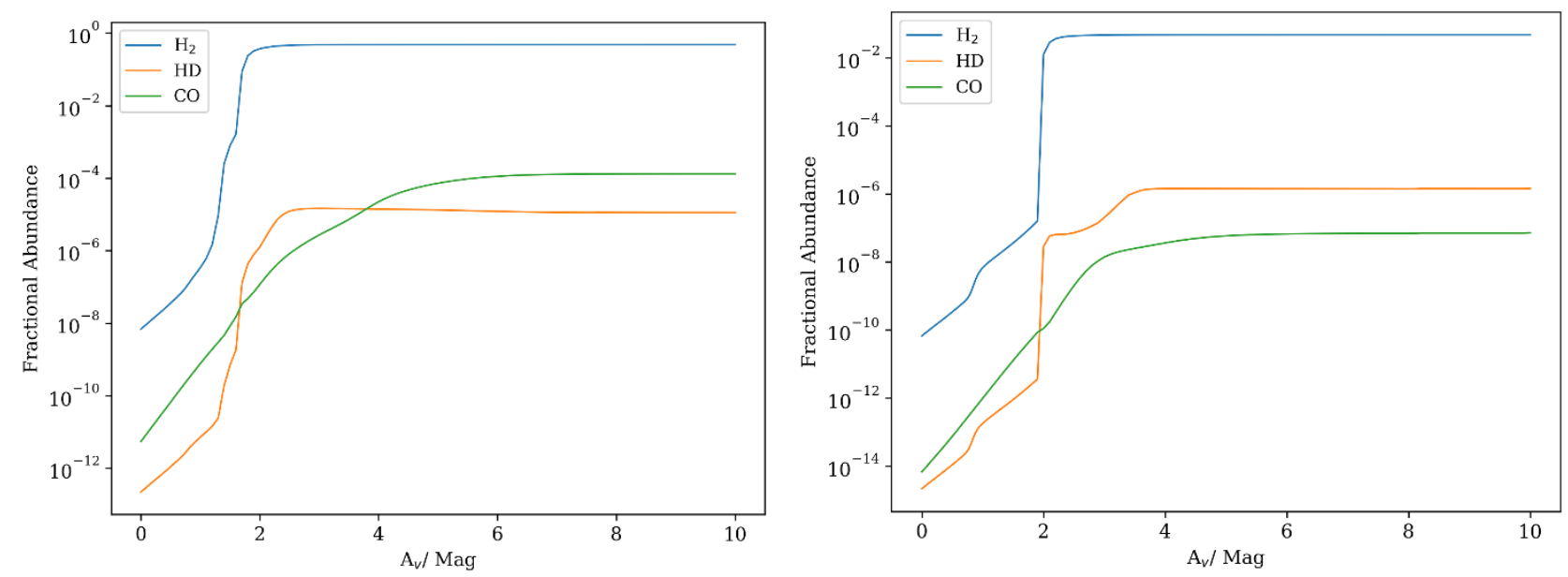

Figure 12. Theoretical predictions of the relative abundance of HD (by S. Viti) with respect to CO and other species as a function of optical depth for molecular gas at a volume density of $10^{4} \mathrm{~cm}^{-3}$ and a metallicity of 1.0 (left) and 0.1 (right) Solar and a radiation field 100 times stronger than the Galactic interstellar field. From the figures one can see that between $\sim 2$ and 4 mags, even for a solar metallicity, $\mathrm{X}(\mathrm{HD})$ can be > than $\mathrm{X}(\mathrm{CO})$. OASIS with its large collecting area and high velocity resolution opens new avenues for direct detection using lines of HD that can only be observed from space.

HD forms via the direct reaction of molecular hydrogen with $\mathrm{D}^{+}$and is mainly destroyed by photo dissociation. It is, therefore, a good tracer of dense molecular gas because at high extinctions (and hence at high densities) HD is well selfshielded. The distribution of HD is expected to closely follow that of $\mathrm{H}_{2}$. Unlike $\mathrm{H}_{2}, \mathrm{HD}$ has a small dipole moment, so dipole transition spectral lines exist. NASA's Far Ultraviolet Spectroscopic Explorer (FUSE) mission detected HD in the Large Magellanic Cloud [55] and tentatively showed that indeed HD does trace $\mathrm{H}_{2}$. Chemical models of dense molecular clouds also show that, above two magnitudes, HD is more abundant by two orders of magnitude with respect to CO (Figure 12).

So far, detecting HD has been a challenge because the lowest transition of HD $(\mathrm{J}=1-0)$ emits in a wavelength range where the dust continuum is bright and the Earth's atmospheric opacity is high (Figure 2). OASIS with its large collecting area and high velocity resolution opens new avenues for direct detection using lines of HD that can only be observed from space.

\subsubsection{OASIS will characterize the evolution of $\mathrm{C}, \mathrm{N}, \mathrm{O}$ and star formation in dusty galaxies from $\mathrm{z}=0 \mathrm{out}$ to $\mathrm{z}=0.8$}

With $O A S I S$ we will follow the trail of water to probe the evolution of hidden black holes and star formation in U/LIRGs, nearby starbursts and AGN and in local low metallicity galaxies, and out to intermediate redshift luminous dusty galaxies. The large collecting area of OASIS also allows for complementary and ground-breaking studies of luminous fine-structure (FS) lines of oxygen, carbon and nitrogen lines in intermediate redshift galaxies. 
Most of the elements we see around us today - carbon, nitrogen, oxygen - were formed in massive stars and released into the ISM at the end of the stellar life cycle. The abundance of metals in a galaxy is a key signature of the galaxy's past history. Gas-phase metallicities represent a direct measure of gas enrichment due to stellar nucleosynthesis and subsequent dispersion of metals in the ISM through stellar winds, supernovae, gas inflow/outflow and, the merger history of a galaxy. Therefore, through the determination of metallicities we are able to place constraints on the star formation (SF) and intergalactic accretion histories of galaxies, and consequently, to constrain models of galaxy formation and evolution. The standard methods to derive gas-phase metallicities are based on UV and optical transitions and are, therefore, susceptible to dust extinction. Such methods are thus not suitable for determining the metallicities of dust-obscured galaxies such as U/LIRGs. Since a large part of the star forming activity in the Universe occurs in dust-obscured environments, extinctionfree metallicity diagnostics are crucial. Far-infrared FS lines provide an alternative way of determining metallicities, avoiding the problem of extinction and uncertain extinction corrrections that plague UV/optical measurements. The FIR regime contains many FS emission lines from various ions and excitation levels that can be used to estimate metallicities in dust-obscured galaxies. Lines such as [O III] $52 \& 88 \mu \mathrm{m}$, [N III] $57 \mu \mathrm{m}$, [N II] $122 \& 205 \mu \mathrm{m}$ arise in H II regions while [C II] $158 \mu \mathrm{m}$ and [O I] $63 \& 146 \mu \mathrm{m}$ lines arise in photo-dissociation regions (PDRs). Lines originating in H II regions probe the ionised component of the ISM and are better suited as tracers of gas phase metallicities as they reflect the metallicity of the gas out of which stars are being formed.
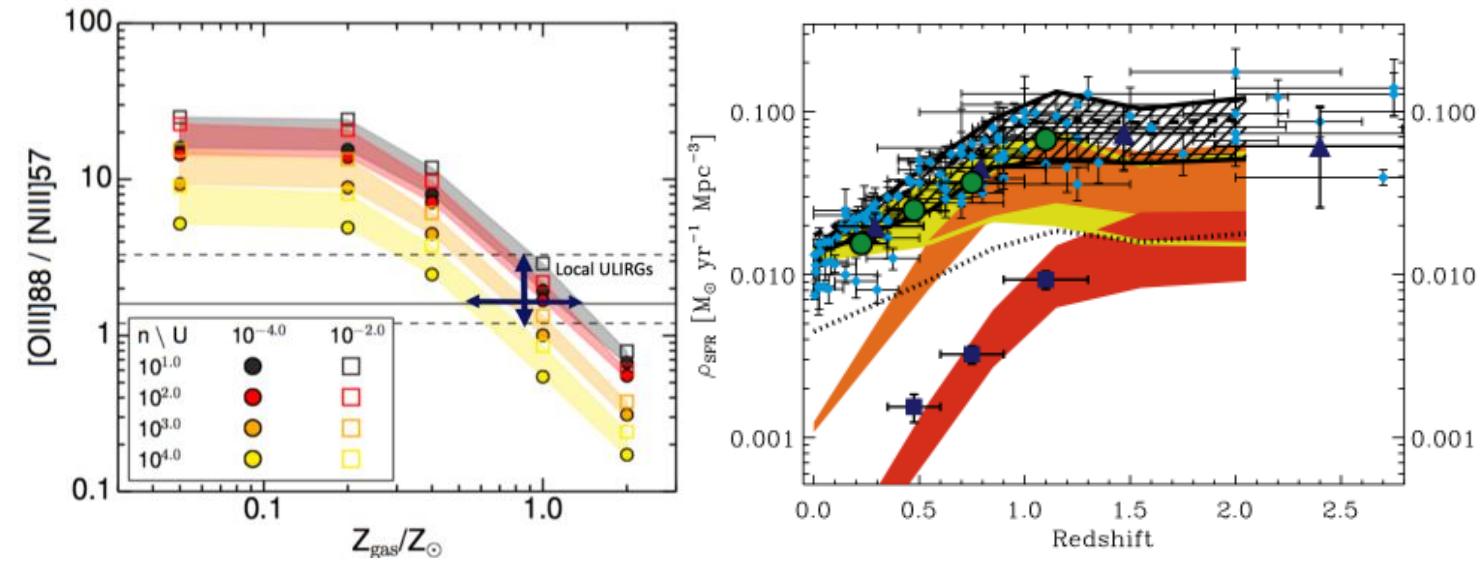

Figure 13. Left: Metallicity estimates for local U/LIRGs (reprinted from [56]); Right: the Star Formation Rate Density of the Universe (SFRD) of the Universe (reprinted from [57]). The shaded region denotes the redshift range probed by OASIS. The redshift range $0<\mathrm{z}<2$ is crucial because this is where a significant change in the SFRD occurs. With OASIS we will have the ability to measure FS lines in a large sample ( 100) of intermediate redshift galaxies probing the last 5 billion years of the Universe when the SF activity slowly decreased to reach the levels we see today.

Pereira-Santaella et al. [56] used combinations of lines ratios of far-infrared FS lines to probe gas-phase metallicities in dusty galaxies (Figure 13, left panel). They found that the most sensitive far-IR line ratios to measure metallicities are those involving the [O III] $52 \mu \mathrm{m}, 88 \mu \mathrm{m}$ and [N III] $57 \mu \mathrm{m}$ lines. These FS line ratios produce robust metallicities even in the presence of an AGN and are insensitive to changes in the age of the ionizing stellar population. The [OIII] 88 $\mu \mathrm{m} /[\mathrm{NII}] 122 \mu \mathrm{m}$ ratio is also suitable to determine the gas phase metallicity [58].

Far-infrared FS lines have been observed in local galaxies with ISO and Herschel [38, 59, 60, 61]. For distant galaxies (z>2) many FIR FS transitions move into the observed submillimeter regime and are now accessible with ALMA [62] However, there is a considerable gap in redshifts between observations of far-infrared FS lines in nearby galaxies with Herschel and detections of FS lines in distant $(z>2)$ galaxies with ALMA. The redshift range $0<z<2$ is crucial because this is where a significant change in the Star Formation Rate Density (SFRD) of the Universe occurs. Indeed, as shown in Figure 13 (right panel), the SFRD of the Universe increases dramatically from the present day to $\mathrm{z} \sim 1$ at which point it reaches a peak out to about $3<\mathrm{z}<4[57,63]$.

With OASIS we will have the ability to measure FS lines in a large sample ( 100) of intermediate redshift galaxies probing the last 5 billion years of the Universe when the SF activity slowly decreased to reach the levels we see today. OASIS is 
the only facility that can access FS lines in the intermediate redshift Universe and probe the ISM of galaxies at an epoch when galaxies wind down the intense SF activity of the cosmic noon to reach the lower SF levels predominant today. In addition, observations of FS lines during that epoch will allow us to chart the metal content of intermediate redshift galaxies and link the local and high-z galaxy populations. OASIS will detect [C II] $158 \mu \mathrm{m}$, [O I] $146 \mu \mathrm{m}$, and [N II] 122 $\mu \mathrm{m}$ lines for galaxies in the redshift range $0.11<\mathrm{z}<0.7$. Additionally, [C II], [O I] $146 \mu \mathrm{m}$, [N II] $122 \mu \mathrm{m}$, and [O III] 88 $\mu \mathrm{m}$ lines will be detected for galaxies in the range $0.54<\mathrm{z}<0.7$.

LIRGS and U/LIRGs are a dominant component of the co-moving SFRD between $0.5<\mathrm{z}<1$, so their properties may give clues to the drivers behind the dramatic rise in SFRD at $\mathrm{z}<1$. Herschel observations of statistically complete samples of intermediate redshift U/LIRGs seem to have shed interesting light on the physical processes that dominate cooling in galaxies near and far. Several studies examined the reliability of FS lines, and in particular of the [C II] $158 \mu \mathrm{m}$ line, as star formation rate indicators [64, 65, 66]. As discussed recently by Dwek and Arendt [67] the [C II] line may suffer from issues in tracing SF in the innermost regions of ULIRGs, but is still a key probe of the global dusty star formation in luminous galaxies. If we are to fully understand the use of FS lines as star forming rate indicators in the distant Universe then it is essential to trace their evolution from present day until redshift 2. OASIS observations of atomic FS lines, and the vital water-molecule transitions, complement each other as essential tracers of the activity and evolution of U/LIRGs, starbursts, AGN, and low-metallicity galaxies.

\section{Contributing authors: S. Aalto, A.A Stark, D. Rigopoulou, S. Viti, L. K., Hunt, and C. Battersby.}




\subsection{OASIS Objective 2: Following the path of water to understand its role in planetary system formation}

\subsubsection{The role of water in protoplanetary disk evolution and planet formation}

Water is key to the emergence of life on Earth. The origin of this water is, however, heavily debated. Water may have been acquired either through the accretion of volatile-rich planetesimals from the colder regions of the Earth's embryonic feeding zone during its build up [68,69], or water may have been delivered to the Earth by the capture of pebbles drifting through the inner solar system on their journey to the Sun [70], or the addition of water may have been a late veneer acquired via impacts of asteroids or comets after planet formation was mostly complete [71,72]. Understanding the distribution of water in protoplanetary disk systems, the processes that regulate its abundance and its evolution, as well as its relationship to the properties of the dust in these disks and the forming planets therein is therefore a key question within astrophysics. OASIS will measure the water content of a broad sample of protoplanetary disks whose structure and properties will have been characterized by ALMA and JWST. The high spectral resolution will allow OASIS to investigate the spatial distribution of water through Doppler tomography using a set of carefully selected lines that probe the wide range of physical conditions inherent to protoplanetary disks. The observed sample of disks will include systems spanning a wide range of ages to measure the time evolution of the water content of protoplanetary disks and trace its role in ongoing planet formation.

Low mass star formation starts with the gravitational collapse of a molecular cloud core to a central protostellar object, which, because of angular momentum conservation, is surrounded by an accretion disk. This system is embedded in an envelope of infalling material [73]. Several stages can be discerned in the subsequent evolution [74]. During the earliest phases - the Class 0 phase -, the protostellar object is deeply embedded in the envelope and still actively accreting mass at a high rate from the envelope through the disk. Some of the accretion energy is converted into a stellar jet and/or disk wind dispersing the reservoir of material in the envelope. As the envelope mass decreases due to ongoing accretion and dispersal by stellar wind, the object will enter the Class I phase, typically after some 100,000-200,000 years. During this late accretion phase, the envelope mass will continue to decrease and eventually the system will enter the Class II phase of an optically visible classical T Tauri star. It is during the Class I and Class II phases that planet formation is thought to occur. Finally, as accretion onto the central star drops and the gas disk dissipates, the system transitions to the Class III phase of a weak line T Tauri star, harboring a nascent planetary systems.

Water greatly increases the solid mass available for planet formation when fully condensed in icy form, and therefore the efficiency of planet formation, in the core accretion picture [75]. Icy grains are expected to settle out of the atmospheres of protoplanetary disks and collide, growing into larger solids. The layer of water ice increases the sticking efficiency of colliding grains, thus enhancing grain growth. As these icy grains settle, they remove water from the disk surface, dehydrating the atmosphere [76,77,78,79] (Figure 14).

The growing solids migrate inward, enhancing the solid surface density at smaller radii, thereby triggering the formation of planetesimals---the building blocks of planets---via, e.g., the streaming instability. The growth and accumulation of icy planetesimals beyond the snow line---the locus where water transitions from icy to gaseous form---fuels the formation of planets: ice giants, gas giants, and even terrestrial-sized icy planets. These planets can migrate inward, within the water snow line, to the habitable zone, bringing along with them their own supply of water. The compact millimeter continuum emission of most protoplanetary disks [80], along with the recognition that many exoplanetary systems are much more compact than our own Solar System and incorporate large solid masses at small orbital distances [81], may reflect the outcome of the inward migration process of both dust grains and planets. In contrast to this formation pathway, other planets may form "dry" within the snow line, without built-in water reservoirs. Icy planetesimals from beyond the snow line (i.e., comets and asteroids) that are "leftover" from the planet formation process may later bombard these planets and deliver water and prebiotic molecules to their surfaces, fostering their habitability.

OASIS offers the opportunity to test the above expectations and measure the progress of disks along these pathways for planet formation, by measuring the distribution of total gas mass and water abundance in disk atmospheres over a range of disk radii. Previous work with the Herschel Space Telescope has revealed tantalizing, but tentative and limited evidence in support of this picture. The Herschel WISH program found that gaseous water emission from protoplanetary disks was difficult to detect $[82,83]$. The large number of water emission upper limits from WISH may imply that the formation of large solids and the accompanying dehydration and migration process is well advanced in protoplanetary disks. OASIS will confirm and quantify the extent of this process with higher sensitivity observations.

One possible interpretation of the low WISH fluxes is that the atmosphere of the outer gaseous disk is highly dehydrated and water is only present in the atmosphere over the compact range of disk radii that produces the millimeter continuum 
emission, i.e., that there is ready exchange between the gas and solid phases of water, with the latter traced by the disk continuum emission. If so, the radial range of gaseous water emission will match the radial range of disk continuum emission and reflect the inward radial migration of icy solids that fuels planetesimal and planet formation. We can test this hypothesis with the spectrally resolved line profiles that will be obtained by OASIS

An alternative interpretation of the low WISH fluxes is that the water in the disk atmosphere has a small filling factor, possibly because it is concentrated in rings similar to those found for the continuum emission from disks [80,84]. While rings in the disk continuum emission can arise from a wide range of phenomena---including those involving planets from Neptune mass to super-Jovian mass, or no planets at all---rings in the gaseous disk would point to the presence of giant planets which are capable of dynamically sculpting the gaseous disk. Thus, spectrally resolved OASIS water line profiles (which trace the radius from which the emission originates) and ALMA images of CO emission (or other tracers) can reveal the masses and orbital radii of any forming planets.

Finally, and most importantly, with $O A S I S$ we will be able to constrain the extent to which water has been concentrated into icy planet-building solids, answering questions raised by the WISH results. These materials are difficult to measure directly, because most of the water ice resides in the cold disk midplane and is locked up in solids the size of pebbles or larger. Solids that large will not produce a significant spectroscopic signature in the infrared. They are, moreover, too cold to emit in the phonon modes (at $45 \mu \mathrm{m}$ and $62 \mu \mathrm{m}$ ), and because they do not overlap significantly along the line of sight to the near- to mid-infrared continuum (3-15 $\mu \mathrm{m})$, they will also not produce shorter wavelength absorption features (at $3 \mu \mathrm{m}, 6.2 \mu \mathrm{m}$, and $11.5 \mu \mathrm{m}$ ). As a result, these solids are effectively "hidden" in the disk midplane. Furthermore, when icy solids grow beyond centimeter size, they are difficult to detect at any wavelength.

Although cold, planet-building water ice is difficult to measure directly, we can infer its mass indirectly, by measuring the total disk gas mass and comparing it with the gaseous water vapor content of the disk. The total mass of water in the disk, in both icy and gaseous form, is expected to be approximately $0.2 \%$ of the total gas mass. By measuring the difference between the expected total water mass and that of the measured water vapor, we can infer the mass in water ice. By further comparing the inferred total mass of water ice with the observed mass in small pebbles, as measured by the millimeter continuum emission of disks, we can infer the mass of water ice that has been converted into larger, planet-building solids that are unobservable directly.

The limited number of cold water vapor detections in protoplanetary disks to date are biased toward the most massive systems $[82,85,86]$. However, protoplanetary disks span a large range of masses and radial extent, with the average disk far more compact than those typically studied. Additional properties such as the spectral type of the central star, the stellar accretion rate, and whether the star is part of a multi-star system, can influence the water abundance and distribution. The large collecting area of $O A S I S$ allows for the first ever statistical study of water in protoplanetary disks spanning a large range of physical properties. Measurements of the water vapor abundance, distribution, and the total disk mass, when performed for a large sample of disks spanning a range of evolutionary ages, will chart out the evolution of the gaseous water content of disks and their progress toward planet building. In many instances the sources will be spatially unresolved, with spectral line profiles providing unique information regarding spatial origin. However, in a handful of disks OASIS will be able to spatially resolve the cold water vapor distribution for the first time.

With the high sensitivity of $O A S I S$, we may also be able to measure the location of the midplane water snow line in disks. In the Solar System, the snow line divides the terrestrial and giant planets, with all of the large planets, the gas and ice giants, located beyond the snow line. Current planet formation models predict that most planets form beyond the snow line (gas giants, ice giants, and possibly smaller planets) and may subsequently undergo radial migration and dynamical scattering $[87,88]$. Small water-rich bodies from beyond the snow line (icy asteroids and comets) are the expected source of water for dry planets that form within the snow line (e.g., Earth). Measurements of the location of the snow line in disks, when compared with exoplanet demographics (orbital radii as a function of mass) will assess the role of the midplane snow line in determining the architecture of planetary systems, as well as illuminate the extent to which other processes alter exoplanetary orbital radii.

These lines of inquiry bear on major questions identified in the Astro2010 Panel Report on Planetary Systems and Star Formation, such as "How do circumstellar disks evolve and form planetary systems?" (p. 152) and "What is the nature of the planet-forming environment?" (p. 175). The observations also directly address specific needs identified in the 2013 NASA Astrophysics Roadmap "Enduring Visions, Daring Quests", which calls for investigations into "where the most basic ingredients of life, such as water and organic molecules, reside in a stellar nursery before they become incorporated into asteroids and comets." They address the "need to directly detect water in protoplanetary disks and map its locations" 
and enact the recommendation that "By studying star systems at different evolutionary stages, we should be able to determine how the distribution of water evolves with time."

OASIS will obtain spectra for protoplanetary disks in nearby star-forming regions spanning a range in evolutionary stage (Class I, II, and flat spectrum sources), disk continuum flux, and stellar accretion rate. The measured line profiles of water and its isotopes, $\mathrm{HD}$, and other gas tracers, will chart out the evolution in the water vapor distribution and gas content of disks, from which we will infer their progress toward planet building. The observations will also measure the location of the midplane water snow line in a subset of disks.

In its first 6 months, OASIS will carry out sensitive spectral scans across all four bands of approximately 12 disks that are representative of the entire sample, quantifying their spectral properties and making any needed modifications to the survey strategy; it will also observe an additional 50 or more disks in specific, well-chosen wavelength settings. Over the entire 1-year mission, OASIS will obtain similar observations of many more disks, including 20 Class I sources with weak molecular envelopes and $~ 100$ Class II and flat spectrum sources.

\subsubsection{OASIS will measure the water abundance in protoplanetary disks across evolutionary stages}

The abundance and distribution of water during planet formation remains poorly constrained, with few detections of water vapor in disks beyond the mid-infrared, which probes only the very surface region of the disk (its atmosphere) within the snow line. OASIS will measure the disk water content at larger radii, as well as the least probed lines originating from near the snowline, potentially closer to the midplane, where most of the mass is located, than those accessible with JWST. With its large collecting area, OASIS will be far more sensitive than Herschel to the cold-to-warm water vapor in the disk atmosphere beyond $1 \mathrm{au}$, or roughly the water snowline (Figure 14). Using the optically thin $\mathrm{H}_{2}{ }^{18} \mathrm{O}$ emission lines, OASIS will measure water vapor abundances for disks ranging in age from less than 1 million years to greater than 10 million years. The large spectral range allows $O A S I S$ to measure water lines spanning a wide range of excitation energies, from 53 to $1729 \mathrm{~K}$, and probe both warm $(100-400 \mathrm{~K})$ water vapor near the snowline and cold $(<100 \mathrm{~K})$ water vapor in the outer disk. Crucially, OASIS will observe the ground state ortho and para water lines, which cannot be observed with any other facility When combined with near-IR detections of hot water vapor in the inner disk atmosphere from JWST, these measurements will provide a complete census of the water vapor in disks.

When combined with its measurements of total gas mass (see Section 2.2.4) OASIS will determine the fraction of the total water content that is in the form of water ice. These results will provide important constraints on the role of water ice in grain growth and on the solid water reservoir available to forming comets and planets.

\subsubsection{OASIS will map the distribution of water vapor in protoplanetary disks across evolutionary stages}

As stated in the Astrophysics Roadmap, mapping the location of water in protoplanetary disks is crucial for understanding the transport of water during planet formation. Because disk rotation follows a Keplerian velocity profile, the radius at which gas emission originates changes the shape of the spectral line profile. The resulting Keplerian broadening of the spectral line profile dominates over other contributions to the line width, such as thermal and turbulent broadening. Thus, high spectral resolution observations of molecular lines in disks can be used to determine the radial location of the emission without having to spatially resolve the disk, a technique known as tomographic mapping. As shown in Figure 15, the velocity offset for emission originating in the inner disk is of order several $\mathrm{km} \mathrm{s}^{-1}$, assuming a disk inclination of 45 degrees, while in the outer disk the velocity offset is much smaller. The spectral resolution of OASIS is $<1 \mathrm{~km} \mathrm{~s}^{-1}$ (Table 2 ), which is easily able to spectrally resolve emission originating in the inner versus outer disk. 


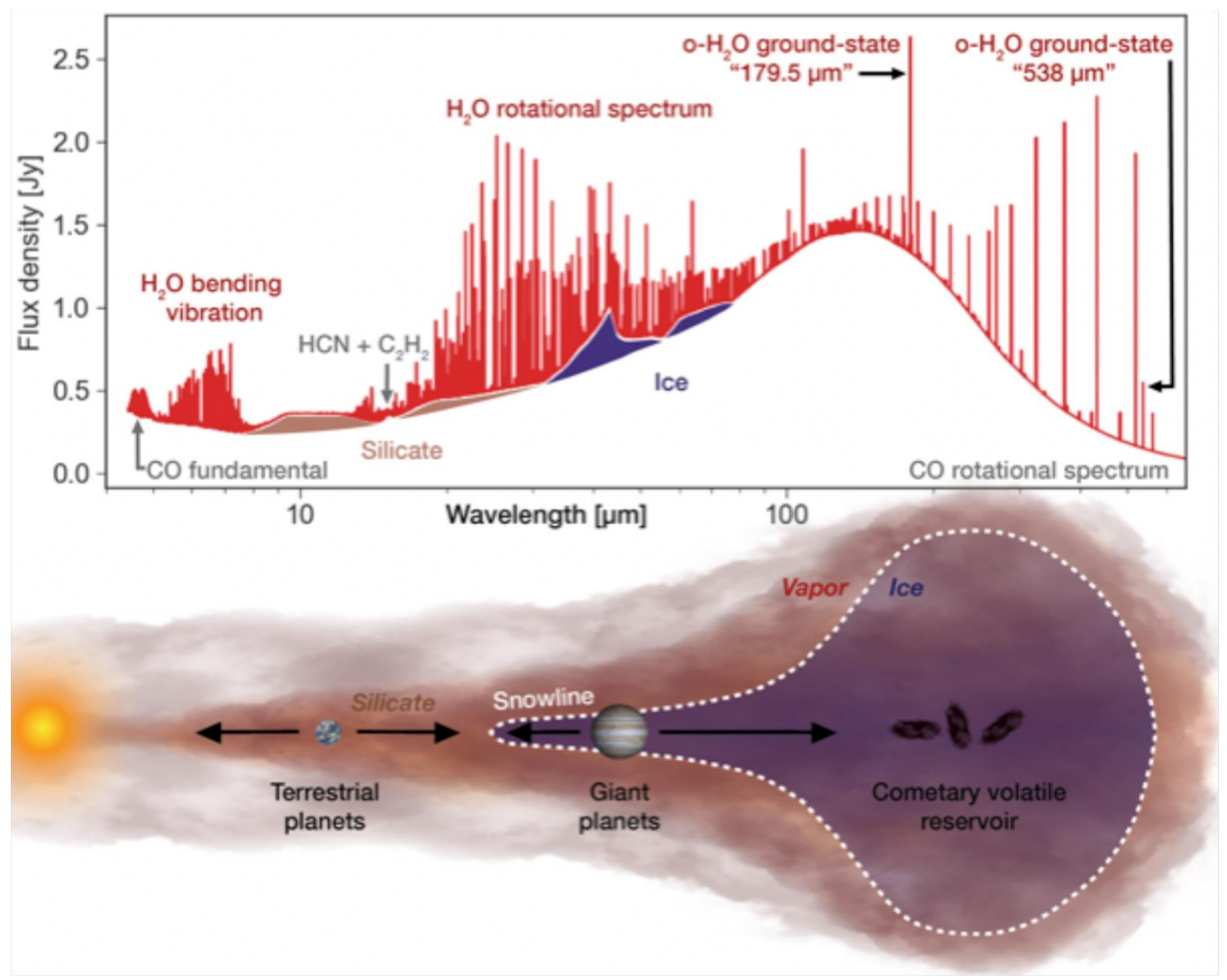

Figure 14. Top: Model broadband water vapor spectrum for a protoplanetary disk. While near IR facilities such as JWST are able to observe high excitation energy water lines, originating from hot (>500 K) gas, OASIS will be sensitive to the ground state ortho and para water lines, which originate primarily from the cold gas at large radii. Bottom: Cartoon cross section of a protoplanetary disk. In the inner disk and the upper layers of the outer disk water is in the gas phase. In the cool, outer regions of the disk, water is frozen out as ice on the surface of dust grains. The transition between water existing primarily in the gas versus the ice is called the water snowline. The midplane water snowline, where planet formation occurs, is thought to be located between the gas giant and terrestrial planet forming regions. Credit: Klaus Pontoppidan/Goddard Space Flight Center

OASIS will use tomographic mapping to determine the radial distribution of water vapor in the brightest protoplanetary disks. These measurements will determine if water vapor is returning to the gas with the inward drift of icy dust grains, enriching the water content of the terrestrial planet forming region. Additionally, OASIS will probe the location of the water snowline by observing multiple water lines with high $(\sim 1000 \mathrm{~K})$ upper state energies, which models predict to emit primarily from inside the water snowline [88,89]. Tomographic mapping will determine the location of the water snowline in systems with a wide range of disk mass and stellar spectral type. These measurements can then be compared to the distribution of rocky and gaseous exoplanets, to assess if the midplane snowline plays a role in the architecture of planetary systems [91,92]. 


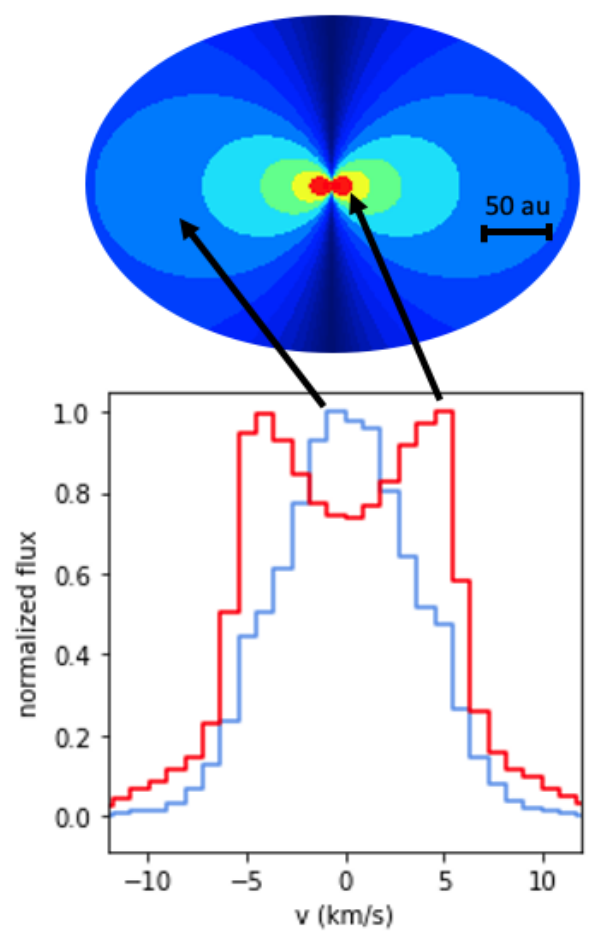

Figure 15. Flux normalized, disk integrated spectra from a protoplanetary disk model inclined 45 degrees from face on, around a 0.8 solar mass star, and at $0.9 \mathrm{~km} \mathrm{~s}^{-1}$ spectral resolution. The ground state $\mathrm{o}-\mathrm{H}_{2} \mathrm{O} 1_{1,0}-1_{0,1}$ emission (blue, $\mathrm{E}_{\mathrm{u}}=$ $61 \mathrm{~K}$ ) originates primarily from the cold outer disk, and thus has a small velocity offset and a centrally peaked spectral line. The o- $\mathrm{H}_{2} \mathrm{O} 63,4-54,1$ emission (red, $\mathrm{E}_{\mathrm{u}}=933.7 \mathrm{~K}$ ) originates primarily from the hot inner disk, resulting in a double peaked spectrum. OASIS will use these differences in the spectral line profile to determine the emitting region for each spectral line.

\subsubsection{Measuring Gas Mass}

Protoplanetary disks — the sites for planet formation — will evolve in mass and size as more mass is accreted from the surrounding core, as angular momentum redistribution within the disk allows accretion onto the central star, and as disk winds return material to the molecular cloud. The disk gas mass is a fundamental property of the disk that regulates its dynamical evolution and its chemical composition. The disk mass also controls the formation of planets through, e.g., coagulation of small dust grains into pebbles that settle to the midplane where planetesimal formation can ensue and gravitational instability of the gas disk leading to a local concentration of mass. Observations reveal a general trend of gas rich disks at early evolutionary stages, with gas-to-dust ratios of $\sim 100$, as seen in the ISM. Over time, the gas mass drops and the gas-to-dust ratio increases until the dust mass exceeds the gas mass, known as the debris disk phase. However, the factors (e.g., disk characteristics, disk age, molecular cloud environment) that control this evolution are not well understood.

Crucially for tracing the water, knowledge of the total disk mass is required to determine the total water (gas+ice) abundance, as chemical abundances are normalized to the hydrogen content. The column density of water vapor can be determined directly from observations of optically thin emission lines, however ice abundances derived from observations of broadband spectral features are rife with uncertainties [93]. An accurate determination of the disk mass is therefore needed to determine how much of the water vapor supplied to the disk has been converted to ice.

The main contributor to the disk gas mass is $\mathrm{H}_{2}$, which does not emit for the majority of regions in the disk because the molecule has no permanent dipole moment and has large energy spacings that are not matched with the local temperatures. The ground-state transition is therefore the quadrupole $\mathrm{J}=2-0$ transition with an energy spacing of $510 \mathrm{~K}$. Thus, exciting an $\mathrm{H}_{2}$ molecule to the $\mathrm{J}=2$ state requires high gas temperatures. $\mathrm{H}_{2}$ emission therefore originates only from the illuminated 
surface layers of the disk within a fraction of an au of the central star. Most of the gas is at larger radii and is much colder. To determine the total gas mass then, alternative tracers must be used.

By far the most commonly used gas mass tracers in protoplanetary disks are continuum emission from dust and emission from rotational transitions of $\mathrm{CO}$. Each method relies on different assumptions. The uncertainties in using dust emission to determine gas mass fall into two categories: assumptions in converting from dust emission to dust mass, and in converting from dust mass to gas mass. Even when the full infrared spectral energy distribution is available, uncertainties in the dust grain optical properties and the grain size distribution lead to significant uncertainty in the derived dust mass from observed emission. Then, to convert from dust mass to gas mass, a gasto-dust mass ratio must be assumed. Typically, this value is taken to be 100, as has been measured in the ISM. However, several factors can change this ratio in disks, including loss of gas due to disk winds and accretion onto the central star, which will decrease the gas-to-dust ratio, and growth of dust grains beyond $\mathrm{cm}$ sizes, at which point the dust emission is no longer observable. This will increase the gas-to-dust ratio.

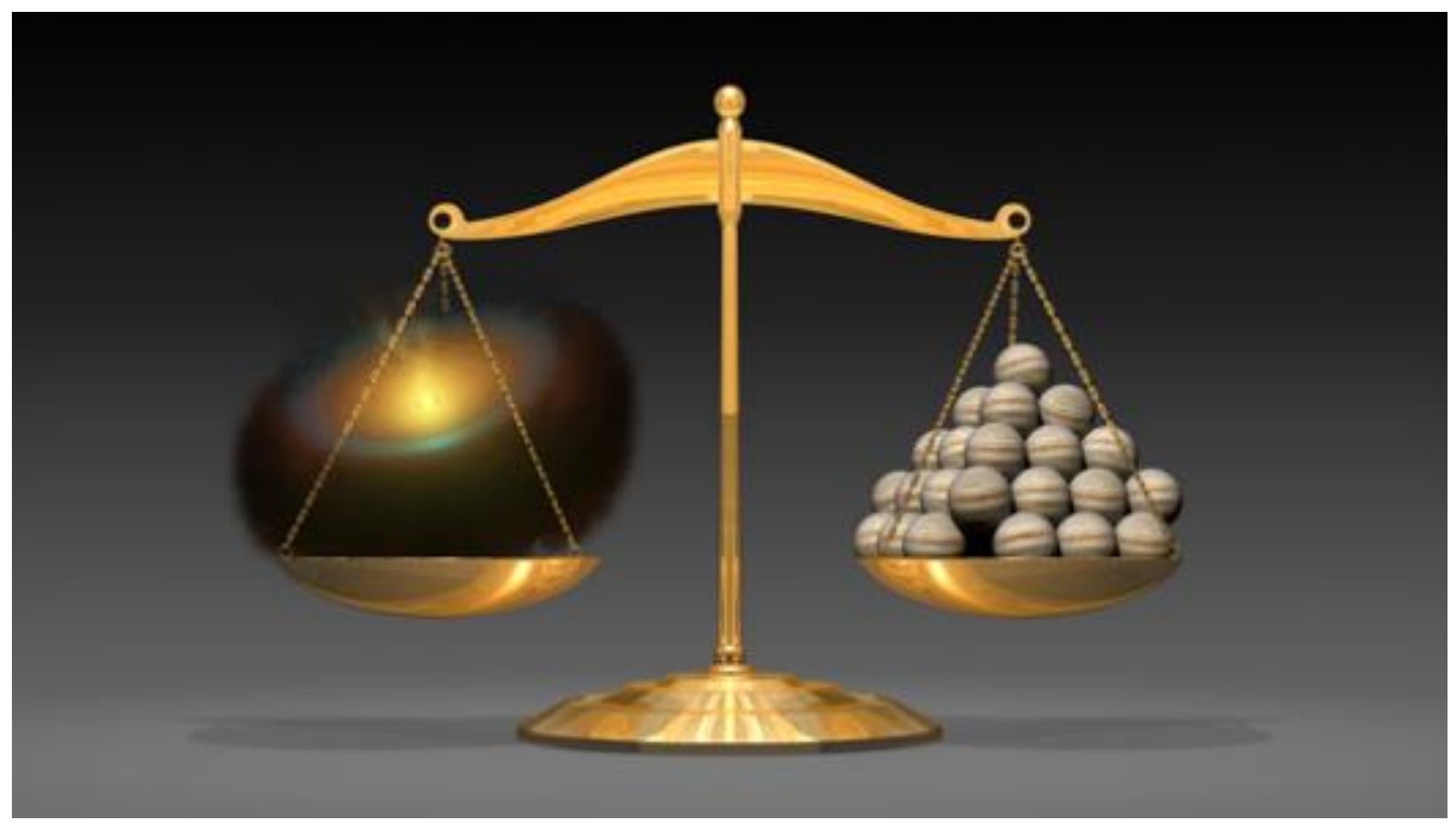

Figure 16. Cartoon scale weighting a protoplanetary disk against a stack of gas giant planets. Credit: Robert Hurt/ JPL IPAC.

Additionally, assuming a constant gas-to-dust ratio is not appropriate. As dust grains grow in size they decouple from the gas, experiencing a headwind which results in a loss of angular momentum and inward radial drift. High spatial resolution observations at millimeter wavelengths demonstrate that the outer radius of the dust disk is often much smaller than the outer radius of the gas disk $[94,95]$.

The $\mathrm{CO}$ abundance relative to $\mathrm{H}_{2}$ in the ISM is well constrained to be $5 \times 10^{-5}$ to $2 \times 10^{-4}$ [96]. However, when converting from $\mathrm{CO}$ abundance to $\mathrm{H}_{2}$ in a protoplanetary disk additional corrections must be made to account for the reduced abundance of $\mathrm{CO}$ relative to $\mathrm{H}_{2}$ in the surface layer, where $\mathrm{CO}$ is photodissociated, and near the cold midplane, where $\mathrm{CO}$ is frozen out onto dust grains [97]. Chemical reactions within the disk also destroy CO [98]. This reduction in the $\mathrm{CO}$ gas abundance varies from disk to disk and as a function of location within a single disk [99], introducing large uncertainties when converting to total gas mass.

Given the myriad assumptions that go into each technique, it is not surprising that the two methods of determining gas mass rarely agree (Figure 17). In fact, most observed disks appear to have significantly less gas than expected from the dust when using $\mathrm{CO}$ as the mass probe. Alternative mass probes, preferably requiring fewer assumptions, are needed to determine the true disk gas mass. One possibility is to use the disk rotation curve to constrain the enclosed mass. This 
technique has recently been demonstrated for a disk with a large total mass of $0.08 \pm 0.04$ solar masses [100]. However, because disks must always be less massive than the central star in order to remain gravitationally stable, the contribution of the disk to the rotation curve is small. Thus, this technique is only feasible for a small number of the most massive disks.

As an exciting new option, the $\mathrm{H}_{2}$ isotopologue $\mathrm{HD}$ can be used to trace disk mass while avoiding many of the limitations of other mass tracers. The HD abundance relative to $\mathrm{H}_{2}$ is well constrained. HD emission is optically thin and not subject to the chemical processing that can change the $\mathrm{CO}$ abundance relative to $\mathrm{H}_{2}$ [96]. However, accurate determination of the gas mass still requires knowledge of the disk temperature structure, as HD does not emit appreciably below $20 \mathrm{~K}$.

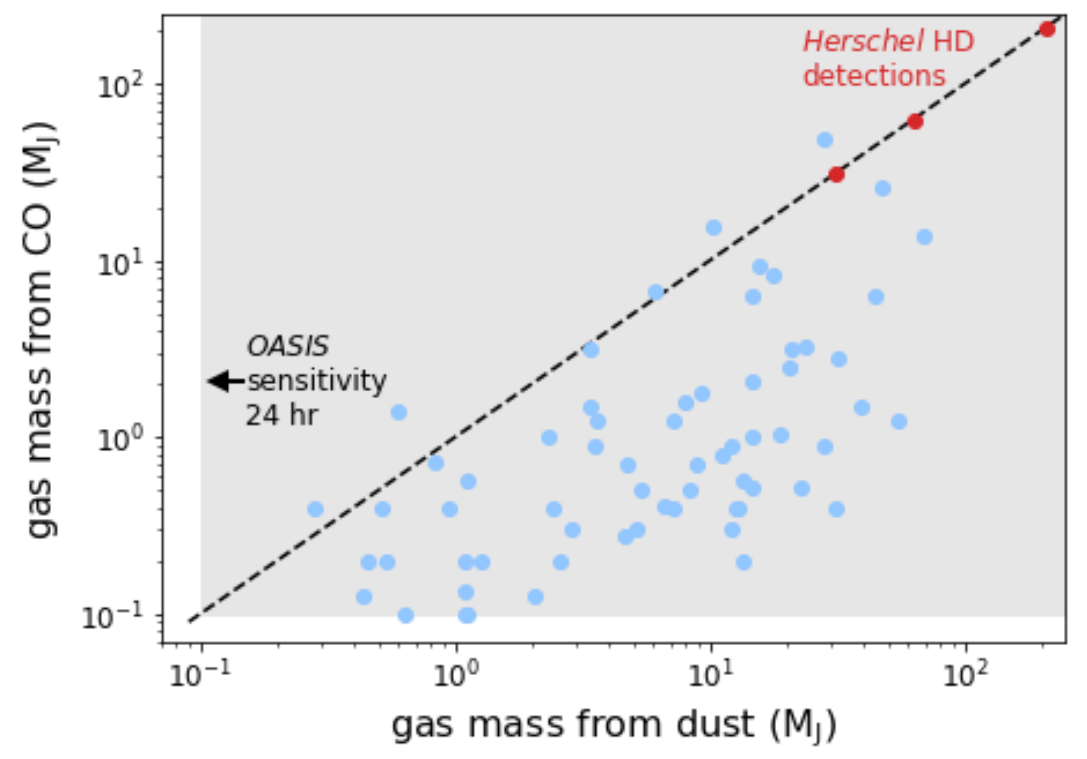

Figure 17. Gas masses derived from millimeter continuum emission versus gas masses derived from rotational $\mathrm{CO}$ transitions for the same systems. The dashed line indicates agreement between the two methods. The CO derived and dust derived gas masses rarely agree, with CO mass estimates usually lower than those based on dust. Additionally, there is a large scatter in the difference between $\mathrm{CO}$ and dust-based mass measurements, suggesting that the amount of $\mathrm{CO}$ and/or dust processing varies from source to source. The grey shaded region shows the mass range OASIS will be sensitive to, assuming a 5 sigma detection on the integrated spectrum with 24 hours on source integration. Conversion from gas mass to HD J=1-0 flux based on HD emission models from [101]. Data from [102, 103, 104, 105].

Near the end of its lifetime, Herschel targeted HD in seven systems, resulting in three detections [105,106]. These were all massive disks, with the HD-derived gas masses of $\sim 30-210 \mathrm{M}_{\mathrm{J}}$. Crucially, these mass measurements reveal that both $\mathrm{CO}$ and $\mathrm{H}_{2} \mathrm{O}$ gas are depleted in these disks relative to the ISM [107, 108]. No current observatory is capable of detecting HD. OASIS will measure the total gas mass in $\sim 100$ protoplanetary systems, down to masses as low as $0.1 \mathrm{M}_{\mathrm{J}}$. When combined with OASIS observations of cold water vapor, this determines the amount of water removed from the outer disk and placed into water ice in the planet forming midplane.

\subsubsection{OASIS will measure disk gas mass in a wide range of protoplanetary systems}

The high sensitivity of $O A S I S$ will allow us to detect HD at a 5 sigma level in disks down to 0.1 Jupiter masses with 12 hours on source, based on model fluxes [98]. This is a factor of 300 more sensitive than what was achieved by Herschel. Converting the HD detections into an accurate total gas mass requires knowledge of the disk temperature structure. The design of OASIS allows for simultaneous observations in the four bands. Thus, while integrating on HD, OASIS will be able to observe multiple $\mathrm{H}_{2}{ }^{16} \mathrm{O}$ and ${ }^{12} \mathrm{CO}$ lines spanning 55-1729 $\mathrm{K}$ in excitation energy, compared to 128.49 for the HD $\mathrm{J}=1$ excited state. These optically thick lines will provide direct measurements of the gas temperature throughout the disk. With OASIS's high spectral resolution, doppler tomography can then be used to map temperatures to a physical radius in the disk. Thus, OASIS observations can be used to obtain measurements of the disk gas masses without the need for ancillary data.

At $2.675 \mathrm{THz}$, the frequency of the HD J =1-0 line, the diffraction-limited beam size of OASIS is $\sim 1.6$ ". The largest protoplanetary disks have gas disks which subtend up to 8 " on the sky. Thus, OASIS will be able to spatially map the HD 
emission in protoplanetary disks for the first time. In unresolved disks with strong HD detections, doppler tomography of the HD spectral line can be used to similar effect. When paired with temperature maps based on $\mathrm{H}_{2} \mathrm{O}$ and $\mathrm{CO}$, this will greatly improve constraints on the temperature of the HD emission and thus result in greater precision when determining the disk gas mass. Additionally, we will be able to map $\mathrm{H}_{2}$ surface density profiles from the HD emission line profile this is completely unique information. Accurate surface density profiles are important for determining dynamics in protoplanetary disks, particularly in the most massive systems, where local over-densities can drive instability, ultimately leading to the formation of massive planets. The gas masses derived from HD will then be combined with the water vapor abundances from $\mathrm{H}_{2}{ }^{18} \mathrm{O}$ to determine the total (gas+ice) water content of protoplanetary disks.

\subsubsection{Astrochemistry with $O A S I S$}

With its broad frequency coverage and tunability, OASIS will cover multitudes of spectral lines from molecular carriers of the main biogenic elements, CHNOPS. In particular, the unprecedented spatial resolution and sensitivity at $\mathrm{THz}$ wavelengths will transform our understanding of the astrochemistry of light hydrides (e.g., $\mathrm{CH}_{\mathrm{x}}, \mathrm{NH}_{\mathrm{x}}$ ) and high-excitation organic molecules. The former is critical for understanding how chemical complexity is built up along the star-formation sequence. The latter is required to assemble a complete picture of the physics and chemistry in hot or shocked interstellar regions.

In the 2010 Astronomy \& Astrophysics Decadal Review [109], 'The Chemistry of the Universe' is listed as a Frontier of Knowledge:

"Study at ever more powerful spectral and spatial resolution of astrophysical environments in which organic molecules occur and evolve is necessary to trace the full potential of organic chemistry to produce molecules of relevance to life, through as much of the galaxy as is possible. Such environments include the interstellar medium, molecular clouds, protoplanetary disks, transition and debris disks, and especially planetary atmospheres."

The OASIS mission directly responds to this goal. First, it will provide spectral coverage of volatile/organic molecule transitions 'for free' alongside the planned observations of $\mathrm{H}_{2} \mathrm{O}$ and $\mathrm{HD}$ in $\sim 100$ protostellar disk systems, enabling robust statistical studies of CHNOPS chemistry in disks. Of particular impact, OASIS will cover multiple strong transitions tracing cool $\mathrm{NH}_{3}$ (upper state energies from 27-170K in OASIS Bands 1 and 2), providing much-needed constraints on the nitrogen budget beyond the snowline in disks. $\mathrm{NH}_{3}$ was detected towards just a single protoplanetary disk with Herschel [110], so there is great potential for discovery science given the significant improvements in sensitivity and spatial resolution offered by OASIS Another promising target in disks is $\mathrm{H}_{2} \mathrm{~S}$, which has multiple lines spanning upper-state energies of $\sim 22-166 \mathrm{~K}$ within OASIS Bands 1 and 2. Sulfur is commonly very depleted from the gas in dense star-forming regions, but $\mathrm{H}_{2} \mathrm{~S}$ was recently detected towards a massive protoplanetary disk [111]. Robust statistics on the occurrence and abundances of $\mathrm{H}_{2} \mathrm{~S}$ in a physically diverse disk sample would provide valuable constraints on the sulfur chemistry in protostars and disks.

Line surveys of additional interstellar environments will provide legacy data sets for the astrochemistry community. In particular, light hydrides and/or high-excitation organics are expected to be key players in the chemistry of photondominated regions [e.g. 112,113], massive hot cores [114,115], diffuse clouds [116,117], and protostellar outflows [118]. OASIS will offer huge improvements in sensitivity and spatial resolution compared to existing observations of these classes of molecules taken with Herschel and SOFIA. Studying the chemistry in these regions allows us to test general principles of how chemical complexity is built up, and how chemistry responds to extreme physical conditions present along certain phases of star and planet formation.

In summary, our understanding of the astrochemistry of prebiotic molecules and their precursors will be greatly expanded by OASIS It will provide (i) statistical constraints on chemical inventories in protoplanetary disks, with areas of particular impact including the nitrogen and sulfur budgets in disks, and (ii) legacy surveys of interstellar regions for which coverage of light hydride and/or high-excitation organic lines are needed to fully understand the chemistry and physics.

\section{Contributing authors: Kamber Schwarz, Jenny Bergner, Yancey Shirely, Joan Najita, Xander Teilens, John Carr, Ted Bergin, David Wilner, Dave Leisawitz.}




\subsection{OASIS Objective 3: Following the path of water to the solar system}

While water is crucial for the emergence of life as we know it, there remain many unresolved questions, for example: How has water evolved from galaxies to protoplanetary disks to our solar system? The OASIS mission will make significant progress towards resolving this question, reinforced by its overarching mission goal, to: Understand how water enables the formation of stellar and planetary systems. Since the ideal planetary system to investigate the role of water is our own solar system, OASIS Science Objective 3 will: Characterize the delivery of water to the solar system by investigating its known water reservoirs. The OASIS Science Objective 3 measurements will answer fundamental, high-priority science questions that stem from both the Astrophysics Decadal Survey [New Worlds, New Horizons in Astronomy and Astrophysics in the decade 2012-2021 [109] and the 2013 NASA Roadmap [Enduring Quests Daring Visions: NASA Astrophysics in the Next Three Decades]. Specifically, Science Objective 3 will address crucial science questions such as: Where does Earth's water come from? This is a key question cited by the Astrophysics Roadmap. Comets and asteroids are believed to have delivered water to the early Earth, and each have distinct $\mathrm{D} / \mathrm{H}$ signatures. OASIS will help determine whether or not comets were the principal sources of Earth's water by measuring the $\mathrm{HDO} / \mathrm{H}_{2} \mathrm{O}$ ratio in both Oort Cloud Comets (OCCs) and Jupiter Family Comets (JFCs).

The Roadmap also advocates completing the reconnaissance of gas giants, terrestrial planets, and Ocean Worlds. OASIS will make significant progress towards this since it will measure $\mathrm{H}_{2} \mathrm{O}$ abundances in the stratospheres of the Gas Giants in order to determine whether planetary rings, icy moons, interplanetary dust particles, or comet impacts deliver water to these planets. Additionally, OASIS will measure the abundance of $\mathrm{H}_{2} \mathrm{O}$ and $\mathrm{HDO}$ in the atmosphere of Venus to better understand how its atmosphere has evolved over time. OASIS will also measure the $\mathrm{H}_{2} \mathrm{O}$ abundance in the torus surrounding Saturn generated by the Ocean World Enceladus, in order to understand how its plume ejecta material (predominately water) has altered the Saturnian environment. Titan, another Ocean World, and the largest satellite of Saturn, has a complex atmosphere containing oxygen compounds that may have been delivered from the $\mathrm{H}_{2} \mathrm{O}$ torus and, ultimately, from Enceladus. OASIS will measure Titan's vertical profile of $\mathrm{H}_{2} \mathrm{O}$ in order to constrain its external source. On Titan, methane plays the role that water does on Earth, with its hydrocarbon lakes, tropospheric methane clouds, and methane precipitation. In addition to water, OASIS will measure $\mathrm{CH}_{3} \mathrm{D}$ and $\mathrm{CH}_{4}$ in order to determine the inventory of deuterium as well as to better understand the methane cycle on Titan. Gaseous $\mathrm{H}_{2} \mathrm{O}$ emission has also been observed from the dwarf planet, Ceres; however, its source and distribution are not known. The high sensitivity of OASIS will allow us to determine whether cryo-volcanism or ice sublimation from localized regions is the source of water. Moreover, the regolith of the Moon contains water ice that may sublimate to form an $\mathrm{H}_{2} \mathrm{O}$ exosphere, and the density and spatial variation of the exosphere is unknown. The unique vantage point of $O A S I S$ at the L1 Lagrange point permits high spatial resolution observations of the Moon, including the south polar region, which is the focus of human exploration. Thus, OASIS will contribute towards both pure science and resource utilization on the Moon, paving the way for future programs like Artemis. This same L1 vantage point will permit detailed observations of the upper atmosphere of Venus. In addition to measuring Venus' D/H, OASIS will observe numerous sulfur compounds, and it will confirm (or refute) the controversial detection of $\mathrm{PH}_{3}$.

In addition to addressing key science questions stated in the NASA Roadmap, OASIS solar system science is also responsive to the Astrophysics Decadal Survey, which cites the following science frontier questions related to origins: What was it about the Sun's nascent environment or its formation that determined the final properties of our solar system versus that of other planetary systems? How much gas and dust was left over for planet formation? OASIS will address these questions by measuring the $\mathrm{HD} / \mathrm{H}_{2}$ ratios in Jupiter, Saturn, Uranus, and Neptune, and these measurements will provide valuable constraints to planetary formation models. This effort will go a long way towards answering the planet formation questions raised by the Decadal Survey.

Solar system science is critical to the $O A S I S$ mission goal as it will focus on the origin and the chemical and physical evolution of water delivered to objects within a planetary system. This, coupled with OASIS ' high spectral resolution, large aperture that enables high sensitivity and high spatial resolution, and its large spectral grasp spanning the submillimeter to far-IR regimes that are largely inaccessible from the ground, will significantly improve upon the accuracy of present-day D/H ratio measurements in both $\mathrm{H}_{2}$ and $\mathrm{H}_{2} \mathrm{O}$. This will be accomplished by observing approximately two dozen solar system objects over the OASIS 1-year baseline mission. The following subsections expand on the justification for conducting highly unique, compelling, and cutting-edge solar system science with OASIS. 


\subsubsection{Why D/H?}

Deuterium fractionation is sensitive to extant conditions (most significantly, temperature) during the epoch of solar system formation. As a result, the $\mathrm{D} / \mathrm{H}$ ratio is a well-established diagnostic for measuring isotopic fractionation in the early solar system. The variation in measured $\mathrm{D} / \mathrm{H}$ ratios for various solar system objects provides important clues to the formation conditions at different locations in the nascent solar system. Of particular interest is how the $\mathrm{D} / \mathrm{H}$ ratio varies in water. The formation process of water involving chemical reactions on interstellar ice grains favors heavier water isotopologues [119, 120]. The $\mathrm{D} / \mathrm{H}$ ratios in the water measured for molecular clouds and protostellar envelopes are approximately $10^{-2}$ and $10^{-3}$, respectively [121,122]. As demonstrated in Figure 18, this is an enrichment of 2 to 3 orders of magnitude over the $\mathrm{D} / \mathrm{H}$ ratio of protosolar hydrogen $\left(2.1 \times 10^{-5} ;[123]\right)$ and in the local interstellar medium $\left(1.6 \times 10^{-5} ;\right.$ [124]). These values may be considered to bracket the $\mathrm{D} / \mathrm{H}$ ratios of water in our solar system, allowing for the possibility of isotopic exchange between water and hydrogen.

Figure 18 shows D/H ratios in water for comets, Enceladus, Earth's oceans (represented by Vienna Standard Mean Ocean Water, or "VSMOW"), carbonaceous meteorites, and star forming regions, and in $\mathrm{H}_{2}$ for the Giant Planets, the ISM, and the protosolar nebula. Care must be taken when comparing the $\mathrm{D} / \mathrm{H}$ ratio in hydrogen and water. $\mathrm{The} \mathrm{D} / \mathrm{H}$ ratio in hydrogen is mainly determined by the Big Bang primordial nucleosynthesis combined with stellar astration [125, 126, 127]. However, the formation process for water requires chemical reactions on interstellar ice grain surfaces at very low temperatures (e.g., $\sim 10 \mathrm{~K}$ ), with highly efficient formation of deuterated water being demonstrated experimentally [128]. In spite of this distinction between hydrogen and water, the range of $\mathrm{D} / \mathrm{H}$ observed within each class of object in Figure 18 suggests a commensurate range of formative conditions.

Given that D/H ratios in water measured for molecular clouds and protostellar envelopes are about 10 to 100 times higher, respectively [121, 122], than VSMOW, the question that arises is: Why is D/H in VSMOW depleted compared with that in molecular clouds and in protostellar envelopes (representing at least partially ISM material)? Is this depletion representative of a chemical memory from a previous evolutionary phase? If so, the relevant processes for this chemical evolution remain unknown. OASIS will make significant progress towards addressing these questions, through measurement of the $\mathrm{HDO} / \mathrm{H}_{2} \mathrm{O}$ abundance ratio in a representative sample of protoplanetary disks and solar system objects. This will establish - at an unprecedented level - the evolution of $\mathrm{HDO} / \mathrm{H}_{2} \mathrm{O}$ from the dark molecular cloud value $\left(\sim 10^{-2}\right)$, to Hot Cores and Hot Corinos $\left(\sim 2 \times 10^{-3}\right)$, to the [unknown] value of mature protoplanetary disks, to values in the present-day solar system.

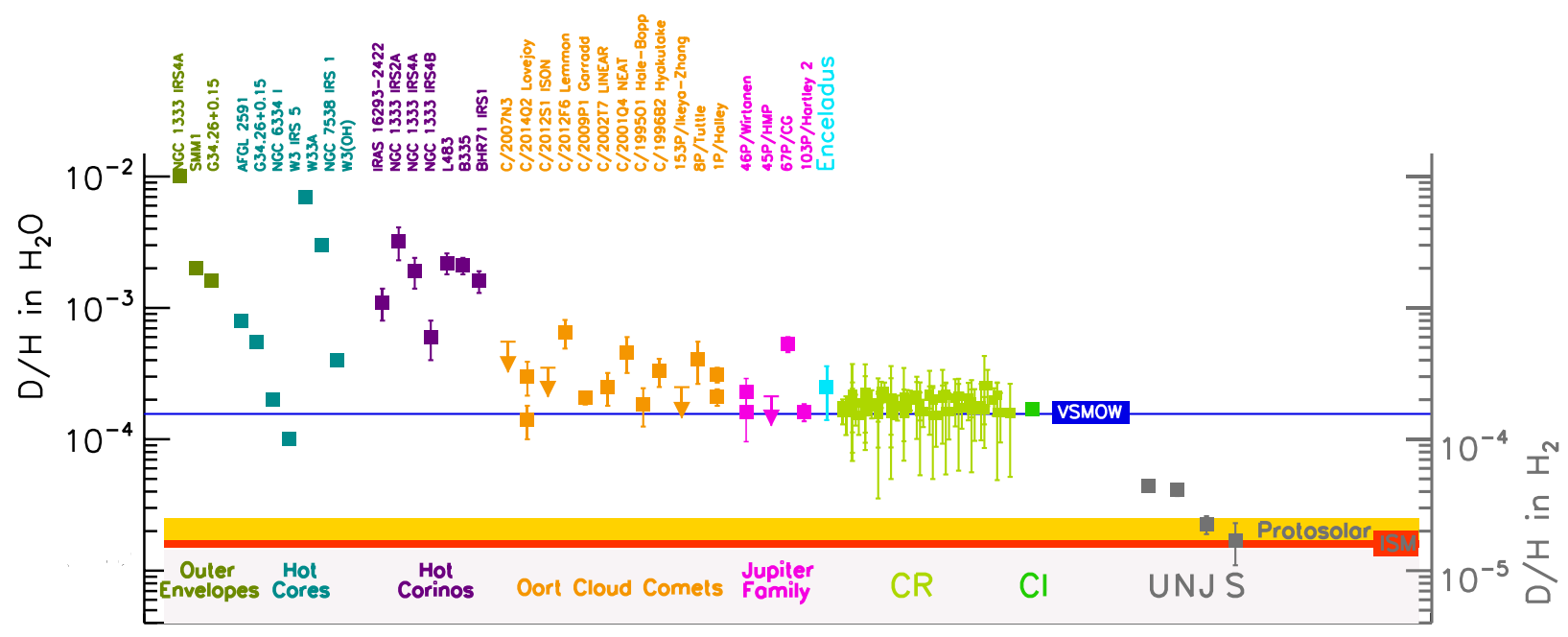

Figure 18. OASIS will measure the $\mathrm{HDO} / \mathrm{H}_{2} \mathrm{O}$ abundance ratio in a representative sample of protoplanetary disks and solar system objects, as well as the HD abundance in the Giants Planets, in which the latter measures the major reservoir of elemental deuterium. D/H ratios for various classes of interstellar and solar system objects are shown. The left ordinate reflects the "cometary" notation for the $\mathrm{D} / \mathrm{H}$ value in water, which is $1 / 2$ the value of $\mathrm{HDO} / \mathrm{H}_{2} \mathrm{O}$. This is only reflected in the values shown for comets, Enceladus, Earth's oceans (VSMOW), and carbonaceous meteorites. The values for the starforming regions (Outer Envelopes, Hot Cores, and Hot Corinos), however, reflect the actual $\mathrm{HDO} / \mathrm{H}_{2} \mathrm{O}$ abundance ratios. Regardless of notation, the $\mathrm{D} / \mathrm{H}$ in water and the $\mathrm{HDO} / \mathrm{H}_{2} \mathrm{O}$ values demonstrate that this [minor] reservoir of deuterium is highly fractionated, as low temperature chemistry greatly enhances small zero-point energy differences between 
isotopologues favoring the deuterated species. The right ordinate gives the $\mathrm{D} / \mathrm{H}$ values in $\mathrm{H}_{2}$ for the Giant Planets, the ISM, and the protosolar nebula, representing the major reservoir of elemental deuterium; as with water, the $\mathrm{D} / \mathrm{H}$ ratio in molecular hydrogen is half the measured abundance ratio $\mathrm{HD} / \mathrm{H}_{2}$. $\mathrm{HDO}$ and $\mathrm{HD}$ are the two main reservoirs for deuterium fractionation. The figure shows that interstellar and solar system objects exhibit large intrinsic dispersions that are well above uncertainties associated with individual measurements, which OASIS will improve upon substantially (e.g., see section 2.3.2). Figure adapted from $[129,130,131]$, with additional inputs from $[4,132,133,134,135,136,137,138,139,140,141,142,143$, $144,145,146,147,148,149,150]$.

Figure 19 illustrates how the origin of water in our solar system may be traced through measurement of the water $\mathrm{D} / \mathrm{H}$ ratio. This figure also sheds some light on the question posed above regarding its relative depletion in VSMOW. In the inner region of the disk (near the Sun) under pressures of $10 \mu$ bar to $1 \mathrm{mbar}$, and temperatures between $600 \mathrm{~K}$ and $1300 \mathrm{~K}$ (e.g., compare to Figure 1 in [149]), within a few hundred years, gaseous water equilibrates with hydrogen, which is at least 4 orders of magnitude more abundant. This means that the $\mathrm{D} / \mathrm{H}$ ratio in water, which was initially highly enriched, will be de-fractionated to its value in molecular hydrogen. With increasing heliocentric distance, temperature and density decrease, as does the efficiency of the isotopic exchange, which stops at an intermediate distance from the Sun where water is still present in gaseous form; this distance is indicated by the "equilibration line" in Figure 19.

Obtaining the most robust value for cometary $\mathrm{D} / \mathrm{H}$ requires measuring the water production rate as near-simultaneously as possible with multiple lines of HDO. This is difficult because as discussed, the most favorable (i.e., strongest) rotational and vibrational lines of $\mathrm{H}_{2} \mathrm{O}$ are not observable from the ground, and for lines that are observable, $\mathrm{H}_{2} \mathrm{O}$ and $\mathrm{HDO}$ are not encompassed simultaneously by any existing ground-based spectrometers. As a result, most estimates rely on the detection of only a single HDO line that was not measured simultaneously with $\mathrm{H}_{2} \mathrm{O}$ (or, in the near-IR, of multiple HDO lines individually having insufficient signal-to-noise ratio; see $[134,135,136])$. Moreover, differing values can be retrieved even for a given comet owing to the use of different techniques by different investigators (as seen for two OCCs and for one JFC in Figure 18). For this reason, a self-consistent dataset has been obtained for only two comets, using the HIFI spectrometer on Herschel: the HDO $509 \mathrm{GHz}$ line was detected quasi-simultaneously with the $\mathrm{H}_{2}{ }^{18} \mathrm{O} 547 \mathrm{GHz}$ and the $\mathrm{H}_{2}{ }^{16} \mathrm{O} 557 \mathrm{GHz}$ lines in one JFC (103P; [129]) and in one OCC (C/2009 P1 (Garradd); [133]). With SOFIA, only a tentative $(3.1 \sigma)$ detection of the HDO $509 \mathrm{GHz}$ line was achieved, albeit near-simultaneously with the $\mathrm{H}_{2}{ }^{18} \mathrm{O} 547 \mathrm{GHz}$ line, in JFC $46 \mathrm{P}$ [137]. This paucity of $\mathrm{D} / \mathrm{H}$ measurements in cometary water will be overcome with OASIS.

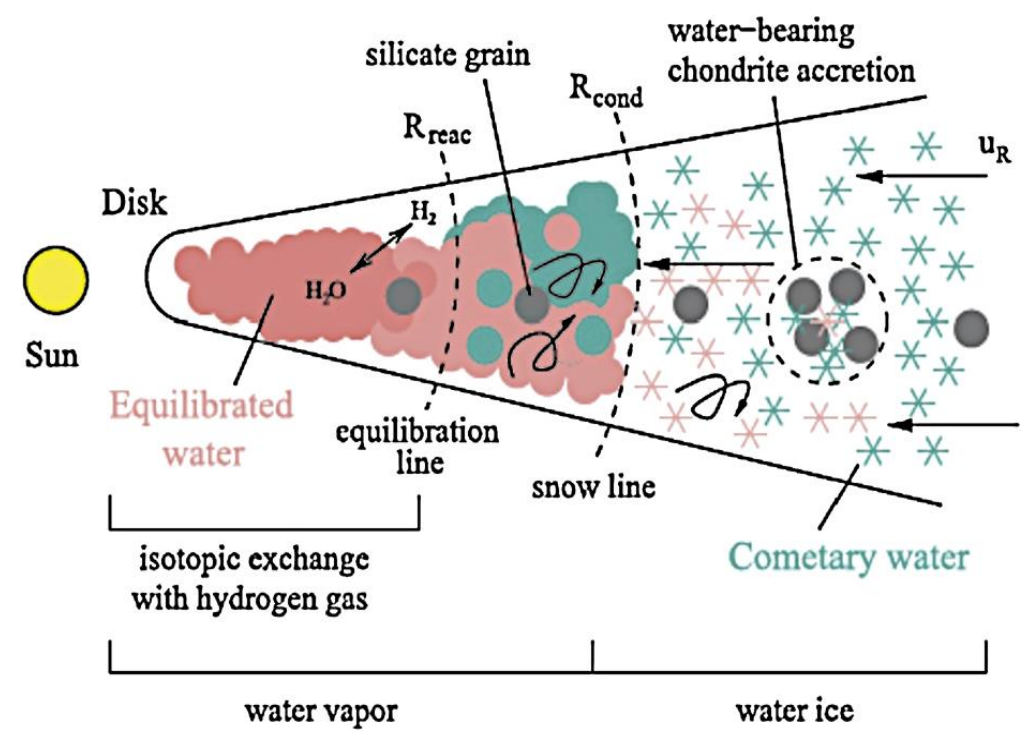

Figure 19. Illustration of the formation of a D/H gradient with heliocentric distance. Arrows symbolize motions of gas (reprinted from [150]). OASIS will permit probing conditions across this gradient.

Vertical mixing of ice grains to the photosphere of the disk becomes very important. The key process is mixing of ice grains to the surface of the disk where photodesorption and photochemistry produces atomic oxygen. This atomic oxygen is then transported downwards again where chemistry reforms it into $\mathrm{H}_{2} \mathrm{O}$ and $\mathrm{HDO}$ on ice grain surfaces but with much reduced deuterium fractionation (see for example [151]). This locates the region where chemistry in the solar nebula can affect the $\mathrm{HDO} / \mathrm{H}_{2} \mathrm{O}$ ratio out to some $50 \mathrm{AU}$ on a timescale of $1 \mathrm{Myr}$. And, since the gas and dust are much warmer than the cold interstellar clouds, the deuterium fractionation of $\mathrm{H}_{2} \mathrm{O}$ will be much smaller. The net result will be a radial gradient 
in the average $\mathrm{HDO} / \mathrm{H}_{2} \mathrm{O}$ ratio (increasing with radius from the central star) and a temporal evolution set by the strength of mixing and chemical timescales involved (decreasing with time).

While Jupiter and Saturn's atmospheric $\mathrm{HD} / \mathrm{H}_{2}$ ratio (within existing uncertainties) reflect the protosolar nebula value $[123,152]$, the $\mathrm{HD} / \mathrm{H}_{2}$ ratios in the Ice Giants (Uranus and Neptune) will be more fractionated [153], reflecting the high ice mass and the importance of these equilibration reactions [151].

While pebbles drifting inward from the cold outer ranges of the solar nebula will contribute relatively pristine presolar ice material with high $\mathrm{HDO} / \mathrm{H}_{2} \mathrm{O}$ ratios to the inner solar system regions, there are many processes that affect the $\mathrm{HDO} / \mathrm{H}_{2} \mathrm{O}$ ratio. These include gas phase chemistry in the warm inner regions of the solar nebula coupled with turbulent mixing outwards, vertical mixing of material coupled with photodesorption, photo-dissociation, and grain surface chemistry, drifting of pebbles with preserved molecular cloud material inwards, and sequestering of material into planetesimals and cometesimals. Although the relative importance of these processes is currently unknown, we do know they will cause a radial and temporal variation in the $\mathrm{HDO} / \mathrm{H}_{2} \mathrm{O}$ ratio. And $O A S I S$, with its dedicated solar system and protoplanetary disk observing program to determine $\mathrm{HDO} / \mathrm{H}_{2} \mathrm{O}$ ratios, is optimized to study these processes in detail.

OASIS will measure this time evolution of the D/H ratio in water and hydrogen by probing a variety of solar system objects, ranging from comets to moons to planets. Collectively, these measurements provide deep insight into the earliest stages of planetesimal formation in the nascent solar system.

\subsubsection{OASIS will measure the HDO abundance in comets}

Since comets are generally considered the most primitive bodies in the solar system, comprised of icy planetesimals left over from planet formation, they are ideal objects to constrain the origin and thermal evolution of water in the solar nebula. As with other classes of objects represented in Figure 18, the D/H values in water for comets show an intrinsic dispersion that is larger than the uncertainties associated with the individual measurements, which for comets span approximately 1 to 3 times that of VSMOW. Given that differences in measurements also exist for individual comets suggests that systematic uncertainties could be significant, perhaps resulting from independent measurements (and retrievals) by different observers. A significant systematic difference in $\mathrm{HDO} / \mathrm{H}_{2} \mathrm{O}$ between dynamical classes may not be the driving factor for these observed differences in the $\mathrm{D} / \mathrm{H}$ values in water. The large spread of values for $\mathrm{D} / \mathrm{H}$ in water suggests that location in the solar system may not be the key parameter in determining the $\mathrm{D} / \mathrm{H}$ value in water, although uncertainties due to the technique used could also factor in. Rather, the water $\mathrm{D} / \mathrm{H}$ value may be controlled by both the radial location where the solar system body was formed as well as when the material was isolated from the vertical mixing to the disk photosphere, thus driving the $\mathrm{D} / \mathrm{H}$ exchange with the gas (see discussion above in Section 2.3.1).

To date, there are measurements of cometary HDO at wavelengths ranging from the UV to sub-millimeter; however, these are relatively few in number. HDO was detected through its vibrational band around $2720 \mathrm{~cm}^{-1}[134,135,136]$, through its rotational lines at $241 \mathrm{GHz}$ [131] and $465 \mathrm{GHz}$ [132, 154], and indirectly via detection of OD [155] or D-Lyman- $\alpha$ (with HST; [156]). The HDO $509 \mathrm{GHz}$ line was searched for once from the ground, but not detected [157]. A different instrument was used for each HDO measurement, and with the exception of Herschel (see below), HDO was not observed simultaneously with other isotopes of water, either in the IR or sub-mm regimes. $\mathrm{HDO}$ and $\mathrm{H}_{2} \mathrm{O}$ were measured in situ (via mass spectrometry), but for only two comets (1P/Halley and 67P/C.G.), during the Giotto and Rosetta space missions, respectively $[158,159]$. As a result, $\mathrm{D} / \mathrm{H}$ ratios in water have been reported in only 16 comets, 4 of which are upper limits and 7 of which were obtained from the ground (Figure 18).

Due to atmospheric opacity, direct ground-based observations of $\mathrm{H}_{2}{ }^{16} \mathrm{O}$ line emission in the near-IR are restricted to weaker (non-fundamental, or "hot") bands in the $\sim 2.8-3.0 \mu \mathrm{m}$ and 4.6-5.0 $\mu \mathrm{m}$ spectral regions (e.g., see [160, 161], and references therein). Also owing to atmospheric opacity, neither has cometary $\mathrm{H}_{2}{ }^{16} \mathrm{O}$ nor $\mathrm{H}_{2}{ }^{18} \mathrm{O}$ been detected in the radio from the ground (and accessible lines are too weak).

Figure 20 shows a rotational diagram of HDO for a simulated observation with OASIS: HDO is not in LTE and it is essential to observe several transitions to retrieve a representative gas rotational temperature. OASIS will observe up to 5 different lines of HDO, with near simultaneous observations of $\mathrm{H}_{2}{ }^{16} \mathrm{O}$ and its $\mathrm{H}_{2}{ }^{18} \mathrm{O}$ and $\mathrm{H}_{2}{ }^{17} \mathrm{O}$ isotopologues - these last two are needed to address opacity issues in $\mathrm{H}_{2}{ }^{16} \mathrm{O}$ lines in more productive comets - to establish precise production rates for both $\mathrm{HDO}$ and $\mathrm{H}_{2} \mathrm{O}$, and hence $\mathrm{D} / \mathrm{H}$ ratios. OASIS will therefore not only greatly increase both the number of comets and the number of water isotopologue lines measured, but will also provide a unique set of measurements using a single observing platform and set of instruments, thereby greatly reducing sources of systematic error associated with previous work represented in Figure 18. 


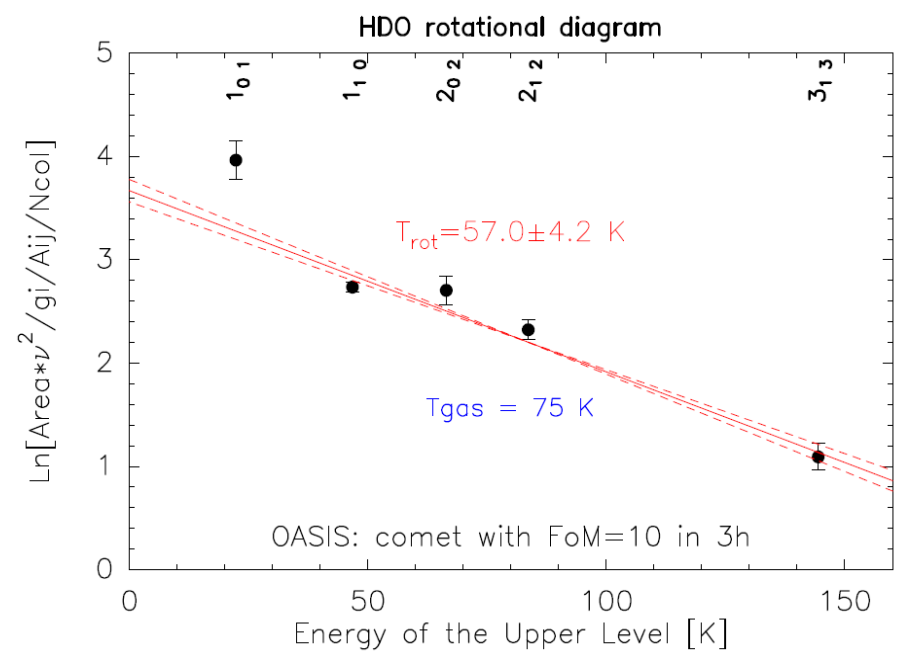

Figure 20. Rotational diagram based on a full non-LTE model simulation for OASIS observations targeting cometary HDO lines at 465, 509, 491, 1278 and $1625 \mathrm{GHz}$ (having upper levels $1_{01}, 1_{10}, 2_{02}, 2_{12}$, and $3_{13}$, respectively, as labeled). Note these lines have energies bracketing the gas temperature. OASIS will measure multiple lines of HDO to derive gas temperatures in comets, and the small beam size (even in Band 1) will reduce uncertainties in gas temperature.

In the OASIS 1-year baseline mission, we estimate 8 - 10 comets will be observed. Figure 21 (left panel) identifies 4 JFCs having favorable apparitions, in which radio Figure-of-Merit (an estimate of spectral line brightness) is shown versus solar elongation angle; OASIS is designed for targets $>45^{\circ}$ from the Sun (including Venus near maximum elongation; see section 2.3.4.2). We also estimate $5-6$ OCCs becoming available for study (Figure 21, right) based on discovery statistics from NEO survey programs over the past two decades that discovered long-period comets as a by-product; these are currently dominated by the Catalina and Pan-STARRS surveys (e.g., [162]).

Additionally, analysis of debiased data from the NEOWISE prime-mission survey [163] suggest that approximately 7 long-period comets (OCCs) that are one km or larger in diameter come within 1.5 AU of the Sun each year [164]. Given the high sensitivity of $O A S I S$, in our estimate of 5-6 OCCs, we expect one or more targets that are sufficiently productive to be characterized even when not at maximum brightness, but when more favorably placed for long duration (multiplehour) observations with $O A S I S$ (i.e., at smaller antisolar angles).
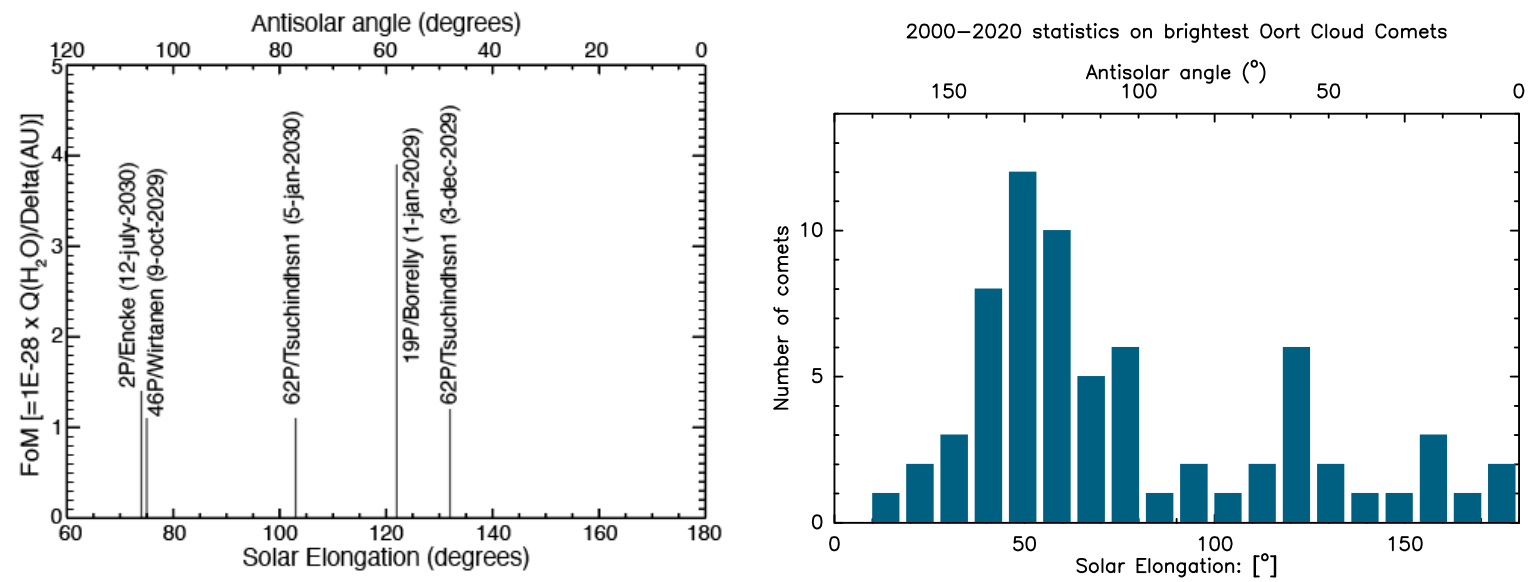

Figure 21. Left: Observing circumstances for 4 JFCs during the OASIS 1-year baseline mission (2029-2030), showing expected radio Figure-of-Merit versus solar elongation angle. Right: 20-year histogram of OCC apparitions representing the brightest comets, $80 \%$ of which had solar elongation $>45^{\circ}$ and thus would be observable by OASIS We expect $5-6$ sufficiently bright OCCs to become available to OASIS during any given 1-year period, based on these statistics. 
Through its uniform set of measurements of $\mathrm{HDO} / \mathrm{H}_{2} \mathrm{O}$, OASIS will therefore greatly improve the accuracy over existing measurements of $\mathrm{D} / \mathrm{H}$ in comets by establishing, in a systematically consistent manner, its value in comets fed from the two principal solar system reservoirs, the scattered Kuiper disk (principal source of JFCs) and Oort cloud (principal source of OCCs). Figure 22 shows a simulation of a comet with the OASIS Bands 1 and 2. Through comparison with $\mathrm{D} / \mathrm{H}$ in Earth's oceans (VSMOW; see Figure 18), cometary D/H measurements constrain the role of comets in delivering water to the young Earth, as well as to other planets. However, as discussed above, measurement of water and its isotopologues from ground-based observations is extremely challenging due to severe extinction by water in Earth's troposphere. With relatively few tuning changes over the course of a comet observing session with OASIS, the strongest transitions of HDO, $\mathrm{H}_{2}{ }^{16} \mathrm{O}, \mathrm{H}_{2}{ }^{18} \mathrm{O}$ and $\mathrm{H}_{2}{ }^{17} \mathrm{O}$ will be measured, thereby establishing highly precise isotopic ratios. Additional molecular species will further constrain the gas temperature in the coma, most notably a suite of $\mathrm{CH}_{3} \mathrm{OH}$ lines around $490 \mathrm{GHz}$.

With its combination of high spectral resolution, high sensitivity, and large spectral grasp enabling near simultaneous observations of multiple $\mathrm{HDO}$ and $\mathrm{H}_{2} \mathrm{O}$ lines, OASIS will accurately measure D/H in water in $8-10$ comets over the OASIS 1-year baseline mission, to investigate the fractionation of water in the proto-planetary nebula.

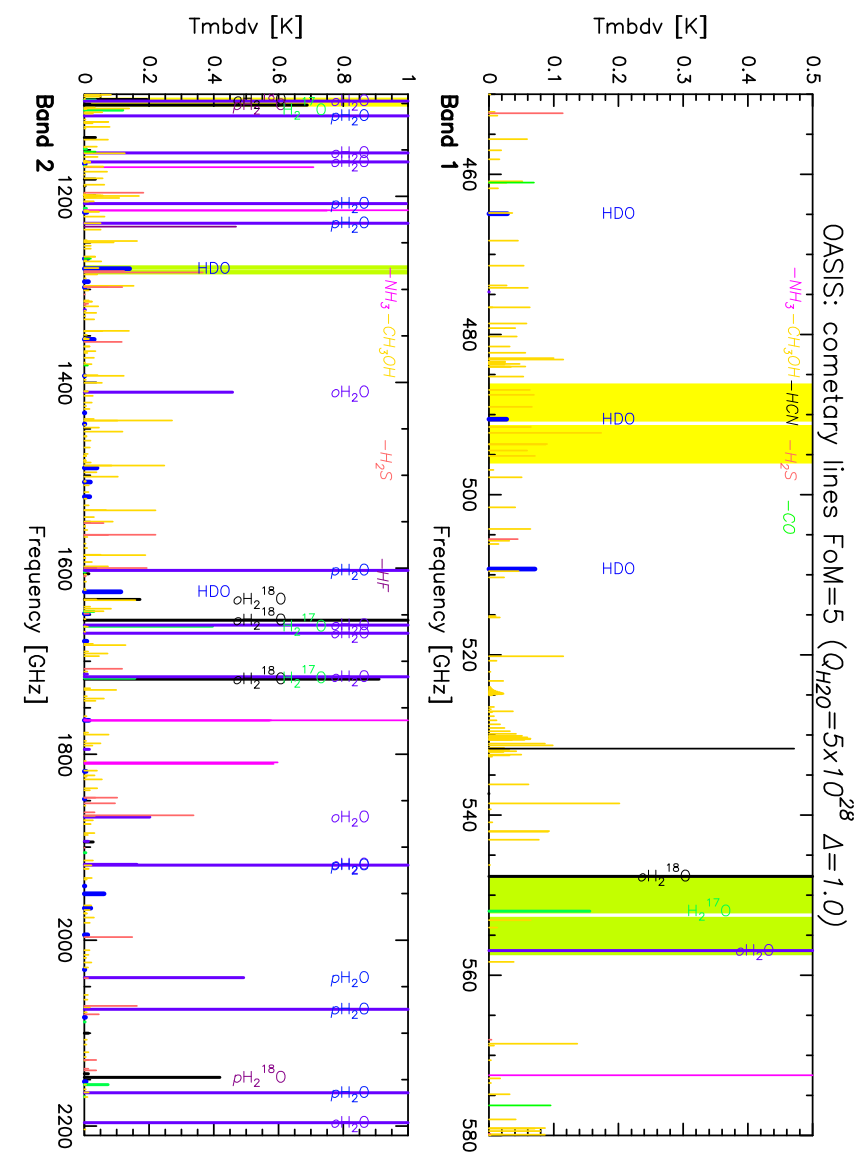

Figure 22. Simulated spectrum of a typical comet observed with OASIS in Bands 1 (upper panel) and 2 (lower panel). The water isotopes are identified and highlighted in thicker lines (light to dark blue). Yellow (green) rectangles show examples of simultaneously encompassed (tunable) frequencies combined in Bands 1 and 2, based on two instrument settings (yellow: 490 and $1099 \mathrm{GHz}$ with one setting; green: 552 and $1277 \mathrm{GHz}$ with a second setting), demonstrating that OASIS will measure $\mathrm{HDO}, \mathrm{H}_{2}{ }^{16} \mathrm{O}, \mathrm{H}_{2}{ }^{18} \mathrm{O}$ and $\mathrm{H}_{2}{ }^{17} \mathrm{O}$ near-simultaneously. 


\subsubsection{Giant Planetary Systems}

\subsubsection{OASIS will measure the water abundance in Enceladus' Torus}

Enceladus is a fascinating small moon of Saturn that is a prime target for investigating the habitability of Ocean Worlds - it may possibly harbor the conditions for the emergence of life. Ocean Worlds like Enceladus, Europa, Titan, and possibly Ganymede, Callisto, and Triton, are active icy moons orbiting planets that reside far outside the classical "Habitable Zone." Instead, these moons are located in the cold outer solar system, where they are expected to be inactive frozen icy worlds. One of the big discoveries stemming from the Cassini 13-year mission in the Saturn system was that Enceladus contained a subsurface liquid water reservoir, which expels hundreds of kilograms of water vapor every second into space through four 2-km wide cracks near its S. polar region $[165,166,167]$. This small but mighty moon is responsible for both of Saturn's tenuous E-ring, as well as the diffuse cloud of water - a torus - around Saturn that extends vertically 10s of thousands of km (see [168] for more details). Both Saturn's E-ring and torus are visible in Figure 23.

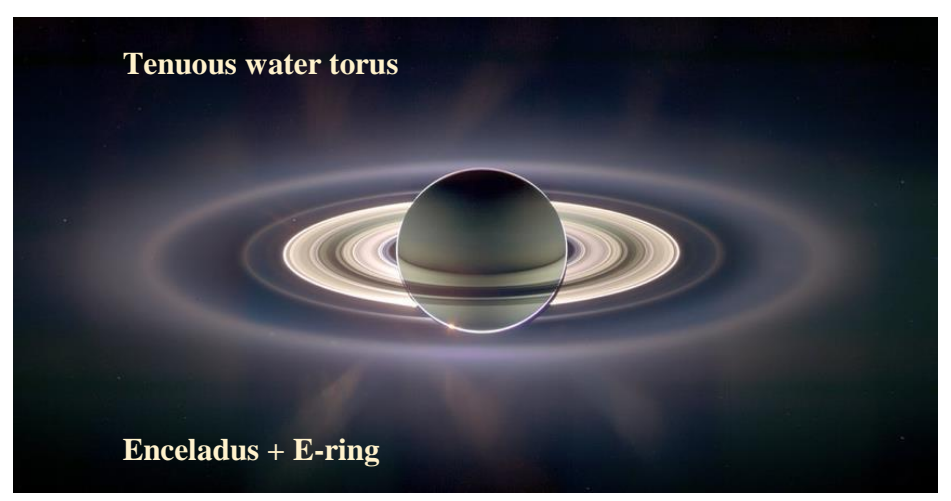

Figure 23. OASIS will measure the spatial variation of the tenuous water torus around Saturn generated by its icy moon Enceladus. Water ice is largely confined to the E-ring in Saturn's equatorial plane, but gaseous water extends tens of thousands of kilometers above the ring plane [168]. Image Credit: NASA/JPL/Space Science Institute.

The HIFI instrument on Herschel discovered the Enceladus water torus by measuring the spatial variation of $\mathrm{H}_{2} \mathrm{O}$ at $1670 \mathrm{GHz}$ [168]. Water was observed in absorption against the disk of Saturn and in emission away from Saturn. The spatial resolution of Herschel at $1670 \mathrm{GHz}$ was 12.6". OASIS will measure this same transition with a spatial resolution of $2 "$ at higher sensitivity. OASIS will also measure multiple lines of $\mathrm{H}_{2} \mathrm{O}$ to derive the gas temperature in the torus. We will expand on the Herschel science by providing a higher spatial resolution map of the torus and by targeting transitions of $\mathrm{HDO}$ and $\mathrm{H}_{2}{ }^{18} \mathrm{O}$ to derive isotopic ratios. Since water originates from a subsurface liquid reservoir, these measurements will provide a rare opportunity to probe conditions in the interior of Enceladus. Due to the small angular size of Enceladus $(0.07 ")$, observing it directly with $O A S I S$ will be extremely challenging since we will suffer from severe beam dilution, even with the small beam size at the high frequency end of Band $4(\sim 1 ")$. In lieu of a direct detection from the plumes themselves, the large spatial expanse of Enceladus' torus offers a unique opportunity to measure the water isotopic ratios for an Ocean World, which are greatly needed to constrain the origin of its water, as well as how it has evolved over time.

To date, there has only been one isotopic measurement of water in Enceladus' plume itself. Cassini INMS obtained a D/H value of $2.9_{-0.7}^{+1.5} \times 10^{-4}$ [169], an enhancement of 1.9 times that in Earth's oceans (see Figure 18). Until another planetary flight mission is sent back to the Saturn System to study Enceladus, OASIS ' high sensitivity is capable of building on both the Cassini and Herschel science discoveries.

With its high spectral resolution and high sensitivity, OASIS will make history by spatially mapping Enceladus' torus, to determine the abundance of water and directly target its emitting isotopologues. This will enable exploring - in detail its physical conditions.

\subsubsection{OASIS will measure the HD abundance in the Giant Planet Atmospheres}

The bulk reservoir for deuterium in the Giant Planets is in the form of HD. And, since there are no known post-Big Bang processes to create deuterium, the deuterium abundances in the Giant Planet atmospheres — measured today — are taken to reflect their primordial value. Accurate measurements of $\mathrm{D} / \mathrm{H}$ in the Giant Planets are needed to constrain models of their origin, but it is difficult to measure HD since this can only be accomplished from space-borne platforms (and airborne 
observatories such as SOFIA). OASIS will measure $\mathrm{HD} / \mathrm{H}_{2}$ in all of the Giant Planets thereby providing the bulk $\mathrm{D} / \mathrm{H}$ ratio. OASIS will also measure stratospheric $\mathrm{H}_{2} \mathrm{O}$ in the Giant Planets (see Section 2.3.3.4), but not HDO; thus, it will not be

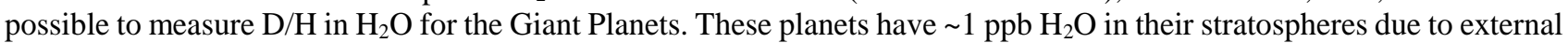
sources discussed in Section 2.3.3.4. The expected abundance of HDO is < 1 ppt and, thus, below the OASIS detection limit. Larger amounts of $\mathrm{H}_{2} \mathrm{O}$ are present at pressures greater than 5 bars in the Giant Planets; however, sub-millimeter observations do not probe deep enough to detect tropospheric $\mathrm{H}_{2} \mathrm{O}$ nor HDO.

In the core accretion scenario for Giant Planets formation [170], highly D-enriched primordial ices formed the planet massive cores onto which the surrounding protosolar $\mathrm{H}_{2}$ gas collapsed. The resulting $\mathrm{HD}$ abundance then results from the ice-to-rock ratio in the core, the core-to-atmosphere mass ratio, and equilibration between the two reservoirs. For instance, the massive $\mathrm{H}_{2}$-dominated atmosphere of Jupiter surpasses by far the mass of its putative core, and $\mathrm{D} / \mathrm{H}$ in Jupiter is therefore thought to reflect the protosolar value ([123] - see Figure 18). For Saturn, however, the measured D/H in hydrogen is smaller than in Jupiter [152], which is unexpected if ice played a role since higher D/H in hydrogen should be expected (as it is the case for the Ice Giants) or perhaps, the ice was equilibrated and transported from the inner to the outer solar system. For the Ice Giants, the D/H is $~ 2$ times higher than the Gas Giants due to a much thinner outer envelope of $\mathrm{H}_{2}$, thereby increasing the weight of the ice-originating D in the final abundance of HD after equilibration [153]. The 410 times lower $\mathrm{D} / \mathrm{H}$ compared with comets remains unexplained. There may be more rocky than icy material in the interior of Uranus and Neptune than expected from formation models [153]. There are various scenarios that could constrain the precise formation position of the Ice Giants with respect to the various snowlines (e.g., [171, 172]). However, error bars on existing measurements of $\mathrm{D} / \mathrm{H}$ in all four outer planets are still large and need to be reduced in order to answer the following questions: Is Saturn's $D / H$ also protosolar? If its $D / H$ is lower than the protosolar value, as indicated by its nominal value measured by ISO and by Cassini/CIRS, what is the mechanism causing this depletion? What is the reason for the intermediate $\mathrm{D} / \mathrm{H}$ in Ice Giants compared with the protosolar value and comet values? OASIS will be able to measure HD in all four Giant Planets, which will significantly improve upon existing HD measurements from ISO, Herschel, and Cassini/CIRS. OASIS will also measure D/H in comets (in water), which are assembled from planetesimals that survived planet formation. OASIS will also measure $\mathrm{D} / \mathrm{H}$ in methane $\left(\mathrm{CH}_{3} \mathrm{D} / \mathrm{CH}_{4}\right)$ in the Ice Giants to further investigate fractionation of deuterium at low temperatures.

Herschel/PACS did not have sufficient spectral resolution to resolve the narrow emission line core originating from the Giant Planet stratospheres. ISO and CIRS also lacked the spectral resolution to separate stratospheric emission from tropospheric absorption, thus limiting the accuracy in deriving $\mathrm{HD} / \mathrm{H}_{2}$. OASIS, however, will easily resolve the narrow emission line core, enabling the derivation of $\mathrm{D} / \mathrm{H}$ in the stratospheres of the Giant Planets; this is depicted for Neptune in Figure 24. HD is expected to be well mixed, but OASIS will be able to test this assumption. OASIS will measure independently $\mathrm{D} / \mathrm{H}$ in the upper troposphere and in the stratosphere and, thus, separate both contributions to the spectrum, similar to what was done for $\mathrm{CO}$ and the deep $\mathrm{O} / \mathrm{H}$ ratio in Neptune $[173,174]$. We will thus better constrain the deep tropospheric $\mathrm{D} / \mathrm{H}$ ratio which is needed to constrain formation processes. The derivation of $\mathrm{D} / \mathrm{H}$ from the emission core of $\mathrm{HD}$ in the Giant Planets requires knowledge of the stratospheric temperature profile. OASIS will measure $\mathrm{CO}$ and $\mathrm{CH}_{4}$ in Band 2 (1.1 to $2.2 \mathrm{THz})$ to obtain the temperature profile for the stratosphere. OASIS also has broad-band continuum channels in each of its 4 bands. These will be used to derive the temperature of the upper troposphere, which is needed to interpret the absorption component of the HD line. OASIS will also have improved spatial resolution $\left(1.4^{\prime \prime}\right.$ at $\left.2.7 \mathrm{THz}\right)$ with respect to Herschel ( 10") and ISO ( 20"). OASIS will be able to resolve the disks of all of the four Giant Planets, thereby improving our knowledge of $\mathrm{D} / \mathrm{H}$. 


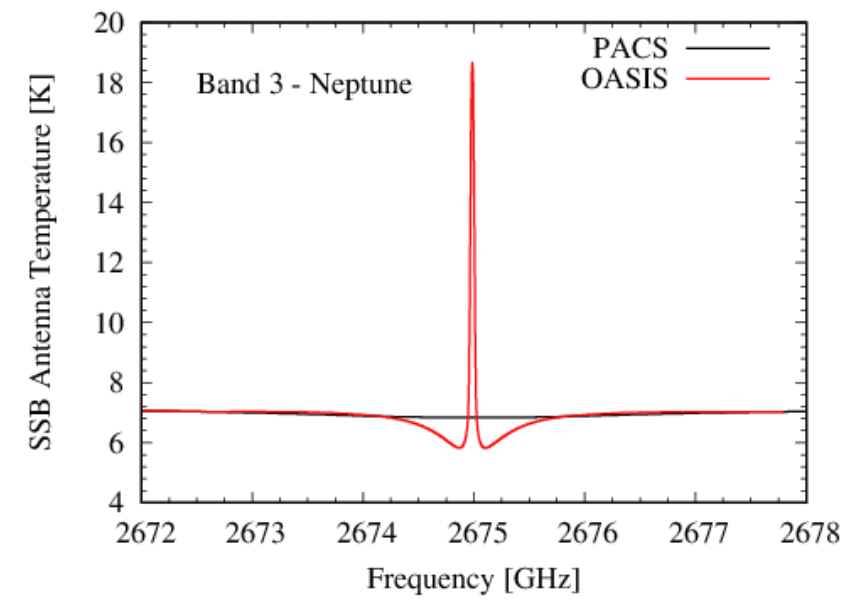

Figure 24. OASIS will use its high spectral resolution to improve the accuracy of $\mathrm{D} / \mathrm{H}$ in the outer planets. Herschel/PACS (black curve) detected a weak HD absorption line on Neptune at a resolving power of 950 [153]. OASIS (red curve) will measure this same feature at more than 100 times this resolution, revealing both stratospheric emission and tropospheric absorption. Resolving the full line shape is critical to determining accurate D/H ratios. (SSB stands for single side band).

OASIS' high spectral resolution, high spatial resolution, and high sensitivity will significantly improve the accuracy of D/H measurements in the Giant Planets. OASIS will be able to measure temperature in Band 2 and HD in Band 3 simultaneously. It will resolve the broad HD absorption line and the narrow emission core, thereby improving the accuracy of known D/H ratios.

\subsubsection{OASIS will constrain the origin of the atmospheres of the Galilean moons and Triton}

The Hubble Space Telescope detected transient $\mathrm{H}_{2} \mathrm{O}$ plumes at Europa in the UV, which were tentatively confirmed with ground-based facilities [175] and from a reanalysis of Galileo data [176]. Based on the water vapor abundance reported by [175], had the Herschel HIFI team been given the observing time on Europa, the HIFI instrument would have easily detected its $\mathrm{H}_{2} \mathrm{O}$ plumes. And, with OASIS' 10-fold enhanced capabilities over that of Herschel, OASIS will be able to confirm the presence of Europa's transient $\mathrm{H}_{2} \mathrm{O}$ plumes, and the corresponding water abundance. Investigating Europa's plume composition with the large spectral coverage and high sensitivity of $O A S I S$ will provide direct access to the composition of the internal ocean. OASIS measurements will precede and so will provide guidance for the arrival of Europa Clipper in 2030.

Herschel found thin spatially variable $\mathrm{H}_{2} \mathrm{O}$ atmospheres at Ganymede and Callisto [177]. It is therefore unknown which process is responsible for maintaining these atmospheres among sputtering, sublimation, hydrothermal activity, or interacting with the icy dust environment of Jupiter. OASIS will conduct repeated observations of $\mathrm{H}_{2} \mathrm{O}$ lines at Ganymede, Callisto, and Europa as a function of their orbital position (e.g., leading vs. trailing), and this will help to constrain the relative role played by these processes and set the stage for the JUICE mission [178, 179].

Voyager 2 detected plumes rising from the surface of Neptune's largest moon, Triton [180, 181]. These are thought to be composed of $\mathrm{N}_{2}$ with entrained condensates. Triton's surface temperature is $38 \mathrm{~K}$, making it unlikely to have gaseous $\mathrm{H}_{2} \mathrm{O}$. However, if Triton has cracks in its ice, analogous to those on Enceladus, warmer temperatures may be possible. OASIS will search for $\mathrm{H}_{2} \mathrm{O}$ outgassing from the surface of Triton. Although unlikely, a detection of $\mathrm{H}_{2} \mathrm{O}$ would radically change our concept of activity on icy moons in the outer solar system, especially at Neptune's distance of 30 AU. OASIS observations will set the stage for in-depth remote sensing and in situ measurements to be carried out by a Neptune-Triton orbiter set for launch in the early 2030s (depending on the outcome of NASA's Planetary Decadal Survey and the selection of either a Flagship or New Frontiers-class mission to the Neptune system).

\subsubsection{OASIS will constrain the external sources of water}

Stratospheric water on Jupiter was first detected from the Kuiper Airborne Observatory immediately following the impact of comet Shoemaker-Levy 9 (SL9) [182]. Shortly thereafter, $\mathrm{H}_{2} \mathrm{O}$ was observed in emission by ISO at millibar levels in the stratospheres of all four Giant Planets and Titan [183, 184]. For the Giant Planets, trace amounts of water were observed in emission at millibar levels in these atmospheres. Water originating from the deep atmosphere is frozen out at the 
tropopause (100 mbar) where temperatures are less than $120 \mathrm{~K}$; thus, the presence of $\mathrm{H}_{2} \mathrm{O}$ in their stratospheres implies an external source.

Water vapor in Titan's stratosphere is also trace, with Cassini/CIRS far-IR nadir and limb sounding measurements revealing a volume mixing ratio of $\sim 0.3 \mathrm{ppb}$ in Titan's stratosphere [185]. As with the Giant Planets, the source of water in Titan's stratosphere is externally delivered, given that water freezes out in Titan's lower stratosphere as a result of vapor condensation processes. Even more so, a positive vertical gradient was derived from the CIRS far-IR [185] and Herschel [186] observations, indicating a source above at higher altitudes and a sink below at lower stratospheric altitudes. This result further reinforces the idea that water is externally delivered to Titan.

OASIS will constrain the role of icy moons in the delivery of species like $\mathrm{H}_{2} \mathrm{O}$ to the upper atmospheres of the Giant Planets and Saturn's largest moon Titan. OASIS will be able to assess the relative contributions of interplanetary dust, icy rings and moons, and large comet impacts by measuring the variation with latitude of $\mathrm{H}_{2} \mathrm{O}$ transitions at 1.670 and $3.691 \mathrm{THz}$, and at spatial resolutions of 2.3 and 1.0", respectively. The former transition is 100 times stronger than the latter at $150 \mathrm{~K}$, while the $3.691 \mathrm{THz}$ line offers better spatial resolution. The smaller beam size (1.0") will roughly match the angular size of Titan ( $\left.0.8^{\prime \prime}\right)$. The use of two lines with different strengths will yield improved vertical profiles of $\mathrm{H}_{2} \mathrm{O}$ in the stratospheres of these objects, which will further constrain its source. In addition, OASIS will also measure the $\mathrm{H}_{2}{ }^{18} \mathrm{O}$ transition at 1.656 $\mathrm{THz}$, thereby providing important isotopic information about the impactors.
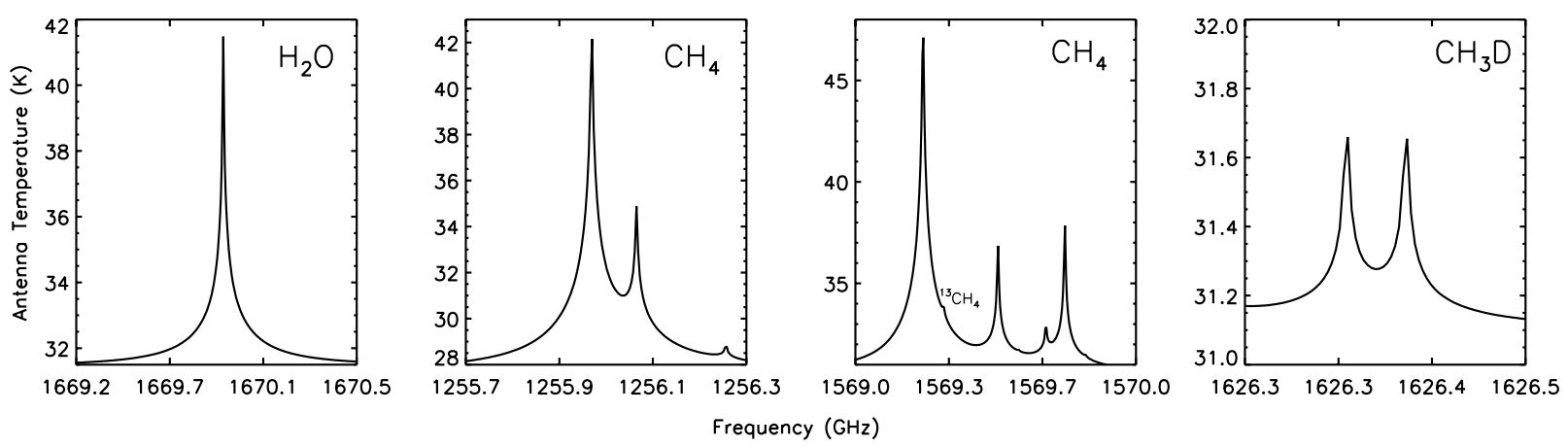

Figure 25. Radiative transfer simulations of the disk-center stratospheric $\mathrm{H}_{2} \mathrm{O}, \mathrm{CH}_{4}$, and $\mathrm{CH}_{3} \mathrm{D}$ emission from Titan at $1.670 \mathrm{THz}, 1.256 \mathrm{THz}, 1.570 \mathrm{THz}$, and $1.626 \mathrm{THz}$, respectively, using the radiative transfer model described in [6]. OASIS' beam size is $\sim 2$ " at these frequencies so Titan's disk (0.8") is spatially unresolved. Spectral resolution is $3.5 \mathrm{MHz}$.

In addition to $\mathrm{H}_{2} \mathrm{O}$, OASIS will also measure $\mathrm{CH}_{4}$ and $\mathrm{CH}_{3} \mathrm{D}$ in Titan stratosphere. While measuring the vertical profile of water in Titan's atmosphere will help to constrain its external source, measuring the vertical profiles of both $\mathrm{CH}_{4}$ and $\mathrm{CH}_{3} \mathrm{D}$ is important as well, given that on Titan, $\mathrm{CH}_{4}$ plays the role that water does on Earth, evidenced by its hydrocarbon lakes, tropospheric $\mathrm{CH}_{4}$ clouds, and $\mathrm{CH}_{4}$ rain (e.g., see [187, 188, 189, 190, 191]). OASIS will measure $\mathrm{CH}_{4}$ and $\mathrm{CH}_{3} \mathrm{D}$ above Titan's cold trap, well above all the tropospheric $\mathrm{CH}_{4}$ weather cycles. Figure 25 shows a forward radiative transfer model simulation of $\mathrm{H}_{2} \mathrm{O}, \mathrm{CH}_{4}$, and $\mathrm{CH}_{3} \mathrm{D}$ emission on Titan at $1.670 \mathrm{THz}\left(\mathrm{H}_{2} \mathrm{O}\right), 1.256$ and $1.570 \mathrm{THz}\left(\mathrm{CH}_{4}\right)$, and 1.626 $\mathrm{THz}\left(\mathrm{CH}_{3} \mathrm{D}\right)$.

High spatial resolution will permit $O A S I S$ to distinguish between possible external sources of water. Interplanetary dust particles (IDP) should impact the Giant Planets from all directions, while local sources such as rings and icy moons would preferentially deliver water to the Equator. For instance, Herschel/PACS measurements of an $\mathrm{H}_{2} \mathrm{O}$ enhancement between $25^{\circ} \mathrm{N}$ and $25^{\circ} \mathrm{S}$ on Saturn favor Enceladus and its $\mathrm{H}_{2} \mathrm{O}$ torus as the dominant source [192]. Cassini/CIRS measurements of $\mathrm{H}_{2} \mathrm{O}$ at the poles of Saturn indicate that there is a smaller component due to IDP [193]. Cassini in situ observations revealed an infall of gases including $\mathrm{H}_{2} \mathrm{O}$ from Saturn's D-ring confined to within $8^{\circ}$ of the Equator [194]. OASIS measurements of $\mathrm{H}_{2} \mathrm{O}$ at the North and South Poles of Jupiter and at the South Pole of Saturn ${ }^{1}$ will reveal the component due to IDP. A detection of enhanced $\mathrm{H}_{2} \mathrm{O}$ over a narrow latitude range at the Equator would favor a ring source and an enhancement over a wider range of latitudes would support delivery from a torus. Higher sensitivity and a large improvement in spatial resolution (1.0-2.3" vs. 9.4" for PACS) will allow OASIS to refine and extend these pioneering measurements.

\footnotetext{
${ }^{1}$ Saturn's North Pole will be in polar night and thus not observable during the OASIS 1-year baseline mission.
} 
Figure 26 shows synthetic spectra of $\mathrm{H}_{2} \mathrm{O}$ emission at $1.670 \mathrm{THz}$ and at $3.691 \mathrm{THz}$ on Jupiter, Saturn, Uranus, and Neptune. Spectrally resolved line profiles at $1.670 \mathrm{THz}$ provide information on the vertical profile of $\mathrm{H}_{2} \mathrm{O}$ in the stratosphere, while the optically thin 3.691-THz feature is sensitive to the total column of $\mathrm{H}_{2} \mathrm{O}$ above the condensation level.
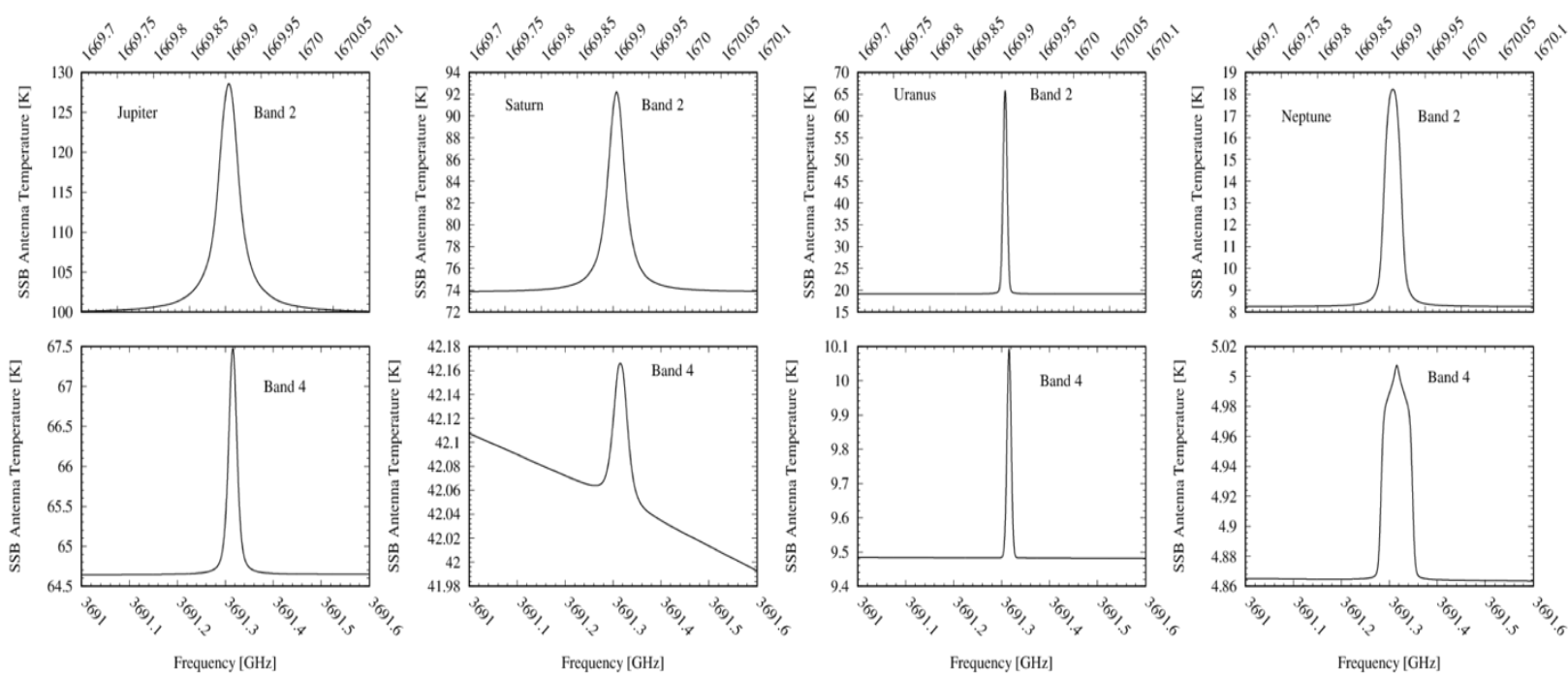

Figure 26. Simulations of the disk-center stratospheric $\mathrm{H}_{2} \mathrm{O}$ emission at $1.670 \mathrm{THz}$ (Band 2) and $3.691 \mathrm{THz}(\mathrm{Band} 4)$ for Jupiter, Saturn, Uranus, and Neptune. SSB stands for Single Side Band.)

Spectrally resolved line profiles will also constrain the external source of $\mathrm{H}_{2} \mathrm{O}$. Herschel/HIFI measurements of the line profile of the 1.670-THz transition of $\mathrm{H}_{2} \mathrm{O}$ on Jupiter indicated that Comet Shoemaker-Levy 9 (SL9), rather than IDP, was the principal source of water [195]. The high spectral resolution of OASIS will extend this approach to the other Giant Planets. New measurements of Jupiter 35 years after the SL9 impact will enable studies of the meridional transport of $\mathrm{H}_{2} \mathrm{O}$ in its stratosphere, thereby constraining dynamical models.

\subsubsection{Inner Solar System}

\subsubsection{Ceres and the Moon}

The presence and abundance of water in asteroids is relevant to many areas of research, ranging from the origin of water and life on Earth to the large-scale migration of Giant Planets such as Jupiter. HIFI observations of water plumes on Ceres with a production rate of about $6 \mathrm{~kg} / \mathrm{s}$ pointed to cryo-volcanism [196]. Later observations of hazes by the DAWN camera however suggest sublimation of water ice over the Occator crater that likely originated from areas beyond the snow line [197]. The initial HIFI observations of Ceres provided ambiguous results with 4s detections in only one polarization and no detection in the other. Repeated observations led to similar results, indicating that the water emission was related to a local source. Finally, 10-hour observations, covering the "light curve" of Ceres led to the crucial detection. The sensitivity of the observation enabled a water production rate of around $1 \mathrm{~kg} / \mathrm{s}$ to be determined. OASIS will increase the sensitivity easily to values below $100 \mathrm{~g} / \mathrm{s}$ water production rate. This high sensitivity opens a new field of Ceres and also asteroidrelated research. For example, water emissions may also be found in other asteroids and in carbonaceous chondrites or main belt comets (MBCs). Water sublimation could be one explanation for the observed dust comae in MBCs, however, all attempts to detect water thus far have been unsuccessful $[198,199]$ due to the limited sensitivity of existing facilities including Herschel/HIFI.

The abundance of water on the Moon is of interest both scientifically and for in situ resource utilization (ISRU) as humans return to the Moon for the first time in a half-century. The first evidence for water ice on the Moon came from observations of neutrons from the Lunar Prospector orbiter [200], where neutron flux spectra were interpreted as providing evidence for hydrogen in the form of water ice at the lunar poles. Direct evidence for hydration on the lunar surface was detected by the Moon Mineralogy Mapper on the Chandrayaan-1 orbiter [201], by the EPOXI mission during a lunar flyby [202], and by Cassini/VIMS during its lunar flyby en route to Saturn [203]. These 3-mm observations showed a mixture of adsorbed water and $\mathrm{OH}$ in the lunar regolith. Next, a plume of water and water ice was detected by the LCROSS investigation following the impact of a Centaur rocket near the lunar south pole [204]. Recently, observations conducted 
from SOFIA detected $\mathrm{H}_{2} \mathrm{O}$ in the regolith at 6 microns at high lunar latitudes [205]. Water generated from meteoroid impacts is expected to form an $\mathrm{H}_{2} \mathrm{O}$ exosphere [206]. The Neutral Mass Spectrometer on the Lunar Atmosphere and Dust Environment Explorer detected exospheric $\mathrm{H}_{2} \mathrm{O}$ liberated by meteoroid impacts [207]. The density and spatial variation of the lunar exosphere is unknown. The unique vantage point of OASIS at the L1 Lagrange point permits high spatial resolution observations of $\mathrm{H}_{2} \mathrm{O}$ and $\mathrm{OH}$ in the exosphere of the Moon, including the polar regions. OASIS will target the $1.670 \mathrm{THz}$ transition of $\mathrm{H}_{2} \mathrm{O}$ and the $1.838 \mathrm{THz}$ line of $\mathrm{OH}$. These observations will help determine whether sublimation of water ice contributes to the exosphere or, in contrast, whether the exosphere is present only following meteoroid impacts.

\subsubsection{Venus}

The upper atmosphere of Venus exhibits large variability in $\mathrm{H}_{2} \mathrm{O}$ on time scales of days [208] to months [209]. Venus is also highly enriched in HDO, with D/H values ranging from 160 times VSMOW in the lower atmosphere to 240 times VSMOW in the upper atmosphere [210, 211]. This has been interpreted as evidence for the loss of a global water ocean due to mass-selective atmospheric escape [210, 213], with important implications for past habitability [213]. The most detailed study of $\mathrm{D} / \mathrm{H}$ was performed using the Solar Occultation Infrared spectrometer on the Venus Express mission [211, 214]. $\mathrm{H}_{2} \mathrm{O}$ and $\mathrm{HDO}$ were measured in absorption against the Sun, at altitudes between 70 and $95 \mathrm{~km}$. The average abundances of $\mathrm{H}_{2} \mathrm{O}$ and $\mathrm{HDO}$ were 1.2 and $0.09 \mathrm{ppm}$, respectively, yielding a D/H enrichment of 240 times VSMOW [211]. The solar occultation geometry limited these measurements to the terminator of Venus. In view of the large variability of water, the ability to map $\mathrm{H}_{2} \mathrm{O}$ and $\mathrm{HDO}$ as a function of latitude and altitude at a wide range of local times would greatly improve our understanding of Venus' atmosphere. Venus' clouds are composed of sulfuric acid; thus, some of the variability in water may be due to reactions with sulfur compounds [208]. Figure 27 shows a radiative transfer simulation of Venus' middle atmosphere for the $1_{10}-1_{01}$ transitions of $\mathrm{HDO}$ at $509 \mathrm{GHz}, \mathrm{H}_{2}{ }^{18} \mathrm{O}$ at $548 \mathrm{GHz}, \mathrm{H}_{2}{ }^{17} \mathrm{O}$ at 552 $\mathrm{GHz}$, and $\mathrm{H}_{2}{ }^{16} \mathrm{O}$ at $557 \mathrm{GHz}$. $\mathrm{HDO}$ and $\mathrm{H}_{2}{ }^{17} \mathrm{O}$ were simulated at $100 \mathrm{kHz}$, which is made possible with OASIS' Chirp Transform Spectrometer. While Figure 27 shows water transitions only in Band 1, numerous transitions exist in Band 2 and Band 4, which also provides higher spatial resolution.
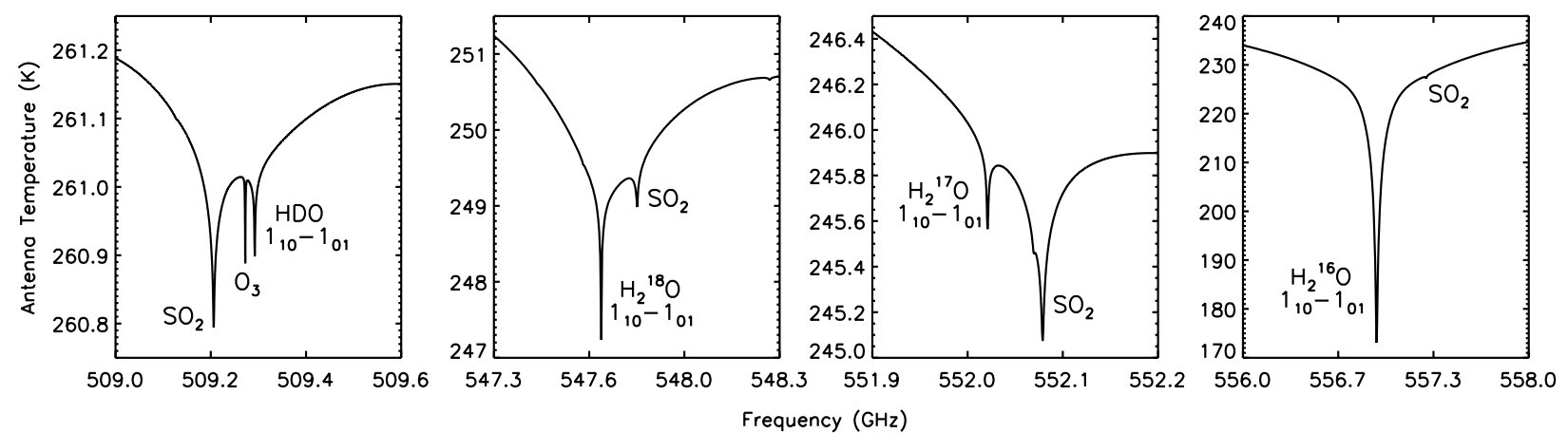

Figure 27. Venus radiative transfer simulations of its disk-center middle atmospheric emission of HDO at $509 \mathrm{GHz}\left(1_{10^{-}}\right.$ $\left.1_{01}\right), \mathrm{H}_{2}{ }^{18} \mathrm{O}$ at $548 \mathrm{GHz}\left(1_{10}-1_{01}\right), \mathrm{H}_{2}{ }^{17} \mathrm{O}$ at $552 \mathrm{GHz}\left(1_{10}-1_{01}\right), \mathrm{H}_{2}{ }^{16} \mathrm{O}$ at $557 \mathrm{GHz}\left(1_{10}-1_{01}\right)$, with an $\mathrm{O}_{3}$ feature and numerous $\mathrm{SO}_{2}$ features in the spectral vicinities. Simulations utilized the radiative transfer model described in [6]. OASIS' beam size is $\sim 8$ " in Band 1. HDO and $\mathrm{H}_{2}{ }^{17} \mathrm{O}$ were simulated at a spectral resolution of $100 \mathrm{kHz}$, while $\mathrm{H}_{2}{ }^{18} \mathrm{O}$ and $\mathrm{H}_{2}{ }^{16} \mathrm{O}$ were at 3.5 MHz.

Sulfur compounds are of interest not only for understanding the clouds, but as tracers of either ongoing volcanism [215], or, alternatively, episodic exchanges with the sulfur-rich lower atmosphere [216]. Thus, it is important to measure $\mathrm{SO}_{2}$, $\mathrm{SO}, \mathrm{OCS}$, and $\mathrm{H}_{2} \mathrm{~S}$ to understand these processes [217, 218]. A detailed study of Venus Express observations of $\mathrm{SO}_{2}$ on Venus indicate large variability and an unknown source of sulfur (other than $\mathrm{SO}_{2}$ and $\mathrm{SO}$ ) between 70 and $100 \mathrm{~km}$ [219]. Recently, ALMA and JCMT observations of Venus led to the unexpected detection of $\mathrm{PH}_{3}$, which was cited as a potential biosignature [220]. Supporting, but not conclusive, evidence for $\mathrm{PH}_{3}$ was provided by a new study of Pioneer Venus Neutral Mass Spectrometer data [221], although this $\mathrm{PH}_{3}$ detection has been disputed by several re-analyses of the submillimeter data $[222,223,224]$ and by its absence in infrared spectra $[225,226]$. As indicated in Figure 28 with OASIS' large spectral grasp in Bands 1 and 2, a total of 5 transitions of $\mathrm{PH}_{3}$ are possible (one is Band 1 and 4 in Band 2) at 534 $\mathrm{GHz}, 1333 \mathrm{GHz}, 1598 \mathrm{GHz}, 1863 \mathrm{GHz}$, and $2128 \mathrm{GHz}$. In view of its significance to both astrobiology and to our understanding of Venus, OASIS will be able to confirm (or refute) the controversial detection of $\mathrm{PH}_{3}$. 

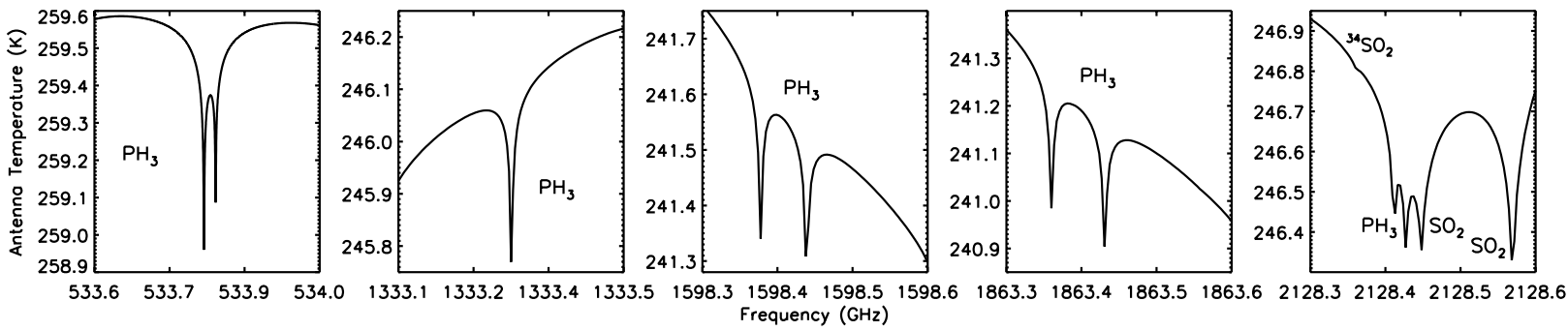

Figure 28. Venus radiative transfer simulations of its disk-center middle atmospheric emission of $\mathrm{PH}_{3}$ at $534 \mathrm{GHz}$, $1333 \mathrm{GHz}, 1598 \mathrm{GHz}, 1863 \mathrm{GHz}$, and $2128 \mathrm{GHz}$. Simulations utilized the radiative transfer model described in [6], assuming a PH3 abundance of 10 ppb. OASIS' beam size is $\sim 8$ " in Band 1 and $\sim 2$ " in Band 2. $\mathrm{PH}_{3}$ in Band 1was simulated at a spectral resolution of $100 \mathrm{kHz}$, while the 4 transitions in Band 2 were at $3.5 \mathrm{MHz}$.

Venus is observable from OASIS while near its greatest elongation from the Sun $\left(47^{\circ}\right)$, at which time it subtends $25^{\prime \prime}$. The tunability of OASIS between 0.465 and $0.557 \mathrm{THz}$ (in Band 1) and between 1.1 and $2.2 \mathrm{THz}$ (in Band 2) will allow us not only to study water and map $\mathrm{D} / \mathrm{H}$ on Venus, but it will enable the study of $\mathrm{PH}_{3}$ and numerous sulfur compounds. OASIS will be able to measure the latitudinal variation and vertical profile of $\mathrm{HDO}, \mathrm{H}_{2} \mathrm{O}$, and sulfur compounds at a wide range of local times on Venus. This will improve our understanding of photochemistry and dynamics in the upper atmosphere. These measurements will build on the successful Venus Express mission, and they will be complementary to in situ measurements from NASA's recently selected DAVINCI+ Discovery mission to Venus.

Contributing authors: Carrie M. Anderson, Michael A. DiSanti, Gordon L. Bjoraker, Nicolas Biver, Thibault Cavalié, Paul Hartogh, Alexander Tielens, and Gordon Chin.

\section{REFERENCES}

[1] Meinel A. and Meinel, M., 2000, "Inflatable membrane mirrors for optical passband imagery," Optical Engineering, $39(2)$.

[2] Clemmons D., 1964, "The Echo I Inflation System", NASA Technical Note TN D-2194. (http://aresinstitute.org/spacepdf/19640012106 1964012106.pdf, https://www.nasa.gov/centers/langley/about/project-echo.html).

[3] Freeland R.E. and G. D. Bilyew, 1992, "IN-STEP Inflatable Antenna Experiment," Acta Astronautica Vol. 30, pp. 29-40.

[4] Jensen, S. S., Jørgensen, J. K., Kristensen, L. E., Furuya, K., Coutens, A., van Dishoeck, E. F., Harsono, D. and Persson, M. V., "ALMA observations of water deuteration: a physical diagnostic of the formation of protostars," Astron Astrophys, 631, A25 (12pp) (2019).

[5] Piccialli, A., Moreno, R., Encrenaz, T., Fouchet, T., Lellouch, E. and Widemann, T., "Mapping the thermal structure and minor species of Venus mesosphere with ALMA submillimeter observations," Astron. and Astrophys, 606, A53 (17pp) (2017).

[6] Villanueva, G. L., Smith, M. D., Protopapa, S., Faggi, S. and Mandell, A. M., "Planetary Spectrum Generator: An accurate online radiative transfer suite for atmospheres, comets, small bodies and exoplanets," JQSRT, 217, 86-104 (2018).

[7] HIFI Observers Manual, 2011, version 2.4, http://herschel.esac.esa.int/Docs/HIFI/html/hifi_om.html.

[8] Walker C. K., 2016, "Terahertz Astronomy", Taylor and Francis Group, Boca Raton, FL.

[9] Sanders, D. B., and Mirabel, I. F., "Luminous infrared galaxies," ARA\&A, 34, 749 (1996).

[10] Casey, C. M., Narayanan, D., and Cooray, A., "Dusty Star-Forming Galaxies at High Redshift,” Phys. Rep., 541, 45 (2014).

[11] González-Alfonso, E., Smith, H. A., Ashby, M. L. N., Fischer, J., Spinoglio, L., and Grundy, T. W., "High-excitation $\mathrm{OH}$ and $\mathrm{H}_{2} \mathrm{O}$ Lines in Markarian 231: The Molecular Signatures of Compact Far-infrared Continuum Sources," ApJ, 675, 303 (2008). S[SEP]

[12] Falstad, N., González-Alfonso, E., Aalto, S., van der Werf, P. P., Fischer, J., Veilleux, S., Meléndez, M., Farrah, D. and Smith, H. A., "Herschel spectroscopic observations of the compact obscured nucleus in Zw 049.057," A\&A, $580,52(2015)$. 
[13] van der Werf, P. P., Isaak, K. G., Meijerink, R., Spaans, M., Rykala, A., Fulton, T., Loenen, A. F., Walter, F., Weiß, A., Armus, L., Fischer, J., Israel, F. P., Harris, A. I., Veilleux, S., Henkel, C., Savini, G., Lord, S., Smith, H. A., González-Alfonso, E., Naylor, D., Aalto, S., Charmandaris, V., Dasyra, K. M., Evans, A., Gao, Y., Greve, T. R., Güsten, R., Kramer, C., Martín-Pintado, J., Mazzarella, J., Papadopoulos, P. P., Sanders, D. B., Spinoglio, L., Stacey, G., Vlahakis, C., Wiedner, M. C., and Xilouris, E. M., "Black hole accretion and star formation as drivers of gas excitation and chemistry in Markarian 231,"A\&A, 518, L42 (2010).

[14] González-Alfonso, E., E.,Fischer, J.,Isaak, K.,Rykala, A.,Savini, G.,Spaans, M.,van der Werf, P.,Meijerink, R.,Israel, F. P.,Loenen, A. F.,Vlahakis, C.,Smith, H. A.,Charmandaris, V.,Aalto, S.,Henkel, C.,Weiß, A.,Walter, F.,Greve, T. R.,Martín-Pintado, J.,Naylor, D. A.,Spinoglio, L.,Veilleux, S.,Harris, A. I.,Armus, L.,Lord, S.,Mazzarella, J.,Xilouris, E. M.,Sanders, D. B.,Dasyra, K. M.,Wiedner, M. C.,Kramer, C.,Papadopoulos, P. P.,Stacey, G. J.,Evans, and A. S.,Gao, Y.,"Herschel observations of water vapour in Markarian 231," A\&A, 518, L43 (2010).

[15] González-Alfonso, E., Fischer, J.,Graciá-Carpio, J.,Sturm, E.,Hailey-Dunsheath, S.,Lutz, D.,Poglitsch, A.,Contursi, A.,Feuchtgruber, H.,Veilleux, S.,Spoon, H. W. W.,Verma, A.,Christopher, N.,Davies, R.,Sternberg, A.,Genzel, R., and Tacconi, L., "Herschel/PACS spectroscopy of NGC 4418 and $\operatorname{Arp} 220: \mathrm{H}_{2} \mathrm{O}, \mathrm{H}_{2}{ }^{18} \mathrm{O}, \mathrm{OH},{ }^{18} \mathrm{OH}, \mathrm{O}$ I, HCN, and $\mathrm{NH}_{3}, "$ A\&A, 541, A4 (2012).

[16] Falstad, N., González-Alfonso, E., Aalto, S and Fischer, J., "Inflowing gas onto a compact obscured nucleus in Arp299A," A\&A, 597, 105 (2017).

[17] Rangwala, N., Maloney, P. R. , Glenn, J., Wilson, C. D. , Rykala, A., Isaak, K., Baes, M., Bendo, G. J. , Boselli, A., Bradford, C. M. , Clements, D. L., Cooray, A. , Fulton, T., Imhof, P., Kamenetzky, J., Madden, S. C. , Mentuch, E. , Sacchi, N. , Sauvage, M., Schirm, M. R. P. Smith, M. W. L, Spinoglio, L., and Wolfire, M. "Observations of Arp 220 Using Herschel-SPIRE: An Unprecedented View of the Molecular Gas in an Extreme Star Formation Environment," ApJ, 743, 94 (2011).

[18] Kamenetzky, J., Glenn, J., Rangwala, N., Maloney, P., Bradford, M., Wilson, C. D., Bendo, G. J., Baes, M., Boselli, A., Cooray, A., Isaak, K. G., Lebouteiller, V., Madden, S., Panuzzo, P., Schirm, M. R. P., and Spinoglio, L., Wu, R., "Herschel-SPIRE Imaging Spectroscopy of Molecular Gas in M82," ApJ, 753, 70 (2012).

[19] Weiß, A., Requena-Torres, M. A., Güsten, R., García-Burillo, S., Harris, A. I., Israel, F. P., Klein, T., Kramer, C., Lord, S., Martin-Pintado, J., Röllig, M., Stutzki, J., Szczerba, R., van der Werf, P. P., Philipp-May, S., Yorke, H., Akyilmaz, M., Gal, C., Higgins, R., Marston, A., Roberts, J., Schlöder, F., Schultz, M., Teyssier, D., Whyborn, N., and Wunsch, H. J., "HIFI spectroscopy of low-level water transitions in M82," A\&A, 521, L1 (2010).

[20] van der Tak, F. F. S., Weiß, A., Liu, L., and Güsten, R., "The ionization rates of galactic nuclei and disks from Herschel/HIFI observations of water and its associated ions," A\&A 593, A43 (2016).

[21] Liu, L., Weiß, A., Perez-Beaupuits, J. P., Güsten, R., Liu, D., Gao, Y., Menten, K. M., van der Werf, P., Israel, F. P., Harris, A., Martin-Pintado, J., Requena-Torres, M. A., and Stutzki, J., "HIFI Spectroscopy of $\mathrm{H}_{2} \mathrm{O}$ Submillimeter Lines in Nuclei of Actively Star-forming Galaxies," ApJ, 846, 5 (2017).

[22] González-Alfonso, E., Smith, H. A., Fischer, J., and Cernicharo, J., "The Far-Infrared Spectrum of Arp 220,” ApJ, 613, 247 (2004).

[23] Yang, C., Gao, Y., Omont, A., Liu, D., Isaak, K.G., Downes, D., van der Werf, P. P., and Lu, N., "Water Vapor in nearby Infrared Galaxies as Probed by Herschel,” ApJ, 771, L24 (2013).

[24] González-Alfonso, E., Fischer, J., Aalto, S., and Falstad, N., "Modeling the $\mathrm{H}_{2} \mathrm{O}$ submillimeter emission in extragalactic sources," A\&A, 567, 91 (2014).

[25] van Dishoeck, E. F., Herbst, E., and Neufeld, D.A., "Interstellar water chemistry: from laboratory to observations," Chem. Rev., 113, 9043 (2013).

[26] Sandford, S. A., and Allamandola, L. J., "The volume- and surface-binding energies of ice systems containing CO, $\mathrm{CO}_{2}$ and $\mathrm{H}_{2} \mathrm{O}$," Icarus, 87, 188 (1990).

[27] Veilleux, S., Maiolino, R., Bolatto, A., and Aalto, S., "Cool outflows in galaxies and their implications," A\&Arv, 28, 2 (2020).

[28] Kroupa, P., Weidner, C., Pflamm-Altenburg, J., Thies, I., Dabringhausen, J., Marks, M., Maschberger, T., "The Stellar and Sub-Stellar Initial Mass Function of Simple and Composite Populations," Proc. Planets, Stars and Stellar Systems Vol. 5, by Oswalt, Terry D.; Gilmore, Gerard, Springer Dordrecht, 115 (2013).

[29] Maeder, A., "Evolution of chemical abundances in massive stars. I. OB stars, Hubble-Sandage variables and WolfRayet stars. Changes at stellar surfaces and galactic enrichment by stellar winds," A\&A, 120, 113 (1983).

[30] Romano, D., Matteucci, F., Zhang, Z.-Y., Papadopoulos, P. P., and Ivison, R. J., "The evolution of CNO isotopes: a new window on cosmic star formation history and the stellar IMF in the age of ALMA," MNRAS, 470, 401 (2017). 
[31] Aalto, S., Muller, S., König, S., Falstad, N., Mangum, J., Sakamoto, K., Privon, G. C., Gallagher, J., Combes, F., García-Burillo,S., Martín, S., Viti, S., van der Werf, P., Evans, A. S., Black, J. H., Varenius, E., Beswick, R., Fuller, G, Henkel, C., Kohno, K., Alatalo, K., and Mühle, S., " The hidden heart of the luminous infrared galaxy IC 860," A\&A, 627, A147 (2019).

[32] Braun, R., Popping, A., Brooks, K., and Combes, F., "Molecular gas in intermediate-redshift ultraluminous infrared galaxies," MNRAS, 416, 2600 (2011).

[33] Hopkins A. M., Beacom J. F., "On the Normalization of the Cosmic Star Formation History,"ApJ, 651, 142 (2006).

[34] Combes, F., Rex, M., Rawle, T. D., Egami, E., Boone, F., Smail, I., Richard, J., Ivison, R. J., Gurwell, M., Casey, C. M., Omont, A., Berciano Alba, A., Dessauges-Zavadsky, M., Edge, A. C., Fazio, G. G., Kneib, J. -P., Okabe, N., Pelló, R., Pérez-González, P. G., Schaerer, D., Smith, G. P., Swinbank, A. M., and van der Werf, P., "A bright z = 5.2 lensed submillimeter galaxy in the field of Abell 773. HLSJ091828.6+514223," A\&A, 538, L4 (2012).

[35] Omont, A., Yang, C., Cox, P., Neri, R., Beelen, A., Bussmann, R. S., Gavazzi, R., van der Werf, P., Riechers, D., Downes, D., Krips, M., Dye, S., Ivison, R., Vieira, J. D., Weiß, A., Aguirre, J. E., Baes, M., Baker, A. J., Bertoldi, F., Cooray, A., Dannerbauer, H., De Zotti, G., Eales, S. A., Fu, H., Gao, Y., Guélin, M., Harris, A. I., Jarvis, M., Lehnert, M., Leeuw, L., Lupu, R., Menten, K., Michałowski, M. J., Negrello, M., Serjeant, S., Temi, P., Auld, R., Dariush, A., Dunne, L., Fritz, J., Hopwood, R., Hoyos, C., Ibar, E., Maddox, S., Smith, M. W. L., Valiante, E., Bock, J., Bradford, C. M., Glenn, J., and Scott, K. S., " $\mathrm{H}_{2} \mathrm{O}$ emission in high-z ultra-luminous infrared galaxies," A\&A 551, A115 (2013).

[36] Yang, C., González-Alfonso, E., Omont, A., Pereira-Santaella, M., Fischer,J., Beelen, A., and Gavazzi, R., "First detection of the $448 \mathrm{GHz}$ ortho- $\mathrm{H}_{2} \mathrm{O}$ line at high redshift: probing the structure of a starburst nucleus at $\mathrm{z}=3.63$," A\&A, 634, L3 (2020).

[37] Jarugula, S., Vieira, J. D., Spilker, J. S., Apostolovski, Y., Aravena, M., Béthermin, M., de Breuck, C., Chen, C., Cunningham, D. J. M., Dong, C., Greve, T., Hayward, C. C., Hezaveh, Y., Litke, K. C., Mangian, A. C., Narayanan, D., Phadke, K., Reuter, C. A., Van der Werf, P., and Weiss, A., "Spatially Resolved Water Emission from Gravitationally Lensed Dusty Star-forming Galaxies at z 3," ApJ, 880, 92 (2019).

[38] Rigopoulou, D., Hopwood, R., Magdis, G. E., Thatte, N., Swinyard, B. M., Farrah, D., Huang, J. -S., Alonso-Herrero, A., Bock, J. J., Clements, D., Cooray, A., Griffin, M. J., Oliver, S., Pearson, C., Riechers, D., Scott, D., Smith, A., Vaccari, M., Valtchanov, I., and Wang, L., "Herschel Observations of Far-infrared Cooling Lines in Intermediate Redshift (Ultra)-luminous Infrared Galaxies," ApJ, 781, 15 (2014).

[39] Smith, R.J., Glover, S.C.O., Clark, P. C., Klessen, R. S., and Springel, V., "CO-dark gas and molecular filaments in Milky Way-type galaxies," MNRAS, 441,1628 (2014).

[40] Smith, R.J., Glover, S.C.O., and Klessen, R. S., "On the nature of star-forming filaments - I. Filament morphologies," MNRAS, 445, 2900 (2014).

[41] Spinoglio, L., Pereira-Santaella, M., Busquet, G., Schirm, M. R. P., Wilson, C. D., Glenn, J., Kamenetzky, J., Rangwala, N., Maloney, P. R., Parkin, T. J., Bendo, G. J., Madden, S. C., Wolfire, M. G., Boselli, A., Cooray, A., and Page, M. J., "Submillimeter Line Spectrum of the Seyfert Galaxy NGC 1068 from the Herschel-SPIRE Fourier Transform Spectrometer," ApJ, 758, 108 (2012).

[42] Wise, J.H., Turk, M.J., Norman,M.L., and Abel, T., "The Birth of a Galaxy: Primordial Metal Enrichment and Stellar Populations," ApJ, 745,50 (2012).

[43] Bullock, J. S., Kravtsov, A. V., and Weinberg., D. H., "Reionization and the Abundance of Galactic Satellites," ApJ, 539, 517 (2000).

[44] Maiolino R., and Mannucci F., "De Re Metallica: The cosmic chemical evolution of galaxies," A\&ArV, 27, 3 (2019).

[45] Joggerst, C. C., Almgren, A., Bell, J., Heger, A., Whalen, D., and Woosley, S. E., "The Nucleosynthetic Imprint of 15-40 M sun Primordial Supernovae on Metal-Poor Stars,” ApJ, 709, 11 (2010).

[46] Acharyya., K., and Herbst, E., "Hot Cores in Magellanic Clouds," ApJ, 859, 51 (2018).

[47] Dickman R. L., Snell R. L., Schloerb F. P., and Solomon P. M., "Carbon Monoxide as an Extragalactic Mass Tracer" ApJ, 309, 326 (1986)

[48] Bryant, P. M., and Scoville, N. Z., "High-Resolution CO Observations of the Ultraluminous Infrared Galaxy Markarian 231,” ApJ, 457, 678 (1996).

[49] Downes, D., and Solomon, P.M., "Rotating Nuclear Rings and Extreme Starbursts in Ultraluminous Galaxies," ApJ, 507, 615 (1998).

[50] Bolatto, A., D., Wolfire, M., and Leroy, A. K., "The CO-to-H2 conversion factor," A\&Arv, 51, 207 (2013).

[51] Cormier, D., et al., "The nature of the interstellar medium of the starburst low-metallicity galaxy Haro 11: a multiphase model of the infrared emission,"A\&A, 548, 20 (2012). 
[52] Madden, S. C., Poglitsch, A., Geis, N., Stacey, G. J., and Townes, C. H., "[C II] 158 Micron Observations of IC 10: Evidence for Hidden Molecular Hydrogen in Irregular Galaxies,” ApJ, 48, 200 (1997).

[53] Bell, T. A. , Hartquist, T. W., Viti, S., and Williams, D. A., "Molecular dark matter in galaxies," A\&A, 459, 805 (2006).

[54] Bisbas, T. G., Papadopoulos, P. P., and Viti, S., "Effective Destruction of CO by Cosmic Rays: Implications for Tracing $\mathrm{H}_{2}$ Gas in the Universe," ApJ, 803, 37 (2015)

[55] André, M. K., et al., "Tiny-scale molecular structures in the Magellanic Clouds. I. FUSE, HST and VLT observations," A\&A, 422, 483 (2004).

[56] Pereira-Santaella, M., Rigopoulou, D., Farrah, D., Lebouteiller, V. and Li, J., "Far-infrared metallicity diagnostics: application to local ultraluminous infrared galaxies," MNRAS, 470, 1218 (2017).

[57] Magnelli, B., Popesso, P., Berta, S., Pozzi, F., Elbaz, D., Lutz, D., Dickinson, M., Altieri, B., Andreani, P., Aussel, H., Béthermin, M., Bongiovanni, A., Cepa, J., Charmandaris, V., Chary, R. -R., Cimatti, A., Daddi, E., Förster Schreiber, N. M., Genzel, R., Gruppioni, C., Harwit, M., Hwang, H. S., Ivison, R. J., Magdis, G., Maiolino, R., Murphy, E., Nordon, R., Pannella, M., Pérez García, A., Poglitsch, A., Rosario, D., Sanchez-Portal, M., Santini, P., Scott, D., Sturm, E., Tacconi, and L. J., Valtchanov, I., "The deepest Herschel-PACS far-infrared survey: number counts and infrared luminosity functions from combined PEP/GOODS-H observations," A\&A, 553, 132 (2013).

[58] Rigopoulou, D., Pereira-Santaella, M., Magdis, G. E., Cooray, A., Farrah, D., Marques-Chaves, R., Perez-Fournon, I., Riechers, D., "On the far-infrared metallicity diagnostics: applications to high-redshift galaxies," MNRAS, 473, 20 (2018).

[59] Cormier, D., Madden, S. C., Lebouteiller, V., Abel, N., Hony, S., Galliano, F., Rémy-Ruyer, A., Bigiel, F., Baes, M., Boselli, A., Chevance, M., Cooray, A., De Looze, I., Doublier, V., Galametz, M., Hughes, T., Karczewski, O. Ł., Lee, M. -Y., Lu, N., and Spinoglio, L., "The Herschel Dwarf Galaxy Survey. I. Properties of the low-metallicity ISM from PACS spectroscopy," A\&A, 578, 53 (2015).

[60] Díaz-Santos, T., Armus, L., Charmandaris, V., Stierwalt, S., Murphy, E. J., Haan, S., Inami, H., Malhotra, S., Meijerink, R., Stacey, G., Petric, A. O., Evans, A. S., Veilleux, S., van der Werf, P. P., Lord, S., Lu, N., Howell, J. H., Appleton, P., Mazzarella, J. M., Surace, J. A., Xu, C. K., Schulz, B., Sanders, D. B., Bridge, C., Chan, B. H. P., Frayer, D. T., Iwasawa, K., Melbourne, J., and Sturm, E., "Explaining the [C II]157.7 $\mu \mathrm{m}$ Deficit in Luminous Infrared Galaxies-First Results from a Herschel/PACS Study of the GOALS Sample,” ApJ, 774, 68 (2013).

[61] Farrah, D., Lebouteiller, V., Spoon, H. W. W., Bernard-Salas, J., Pearson, C., Rigopoulou, D., Smith, H. A., González-Alfonso, E., Clements, D. L., Efstathiou, A., Cormier, D., Afonso, J., Petty, S. M., Harris, K., Hurley, P., Borys, C., Verma, A., Cooray, A., and Salvatelli, V., "Far-infrared Fine-structure Line Diagnostics of Ultraluminous Infrared Galaxies," ApJ, 776, 38 (2013).

[62] De Breuck, C., Weiß, A., Béthermin, M., Cunningham, D., Apostolovski, Y., Aravena, Manuel, Archipley, M., Chapman, S., Chen, C., Fu, J., Jarugula, S., Malkan, M., Mangian, A. C., Phadke, K. A., Reuter, C. A., Stacey, G., Strandet, M., Vieira, J., and Vishwas, A., "A dense, solar metallicity ISM in the $\mathrm{z}=4.2$ dusty star-forming galaxy SPT 0418-47," A\&A, 631, 167 (2019).

[63] Bouwens, R. J., Illingworth, G. D., Franx, M., Chary, R.R., Meurer, G.R., Conselice, C.J., Ford, H., Giavalisco, M., van Dokkum, P., "UV Continuum Slope and Dust Obscuration from z $\sim 6$ to $\mathrm{z} \sim 2$ : The Star Formation Rate Density at High Redshift," ApJ, 705, 936 (2009)

[64] De Looze, I., Cormier, D., Lebouteiller, V., Madden, S., Baes, M., Bendo, G. J., Boquien, M., Boselli, A., Clements, D. L., Cortese, L., Cooray, As., Galametz, M., Galliano, F., Graciá-Carpio, J., Isaak, K., Karczewski, O. Ł., Parkin, T. J., Pellegrini, E. W., Rémy-Ruyer, A., Spinoglio, L., Smith, M. W. L., and Sturm, E., "The applicability of farinfrared fine-structure lines as star formation rate tracers over wide ranges of metallicities and galaxy types," A\&A, 568, 62 (2014).

[65] Magdis, G. E., Rigopoulou, D., Hopwood, R., Huang, J. -S., Farrah, D., Pearson, C., Alonso-Herrero, A., Bock, J. J., Clements, D., Cooray, A., Griffin, M. J., Oliver, S., Perez Fournon, I., Riechers, D., Swinyard, B. M., Scott, D., Thatte, N., Valtchanov, I., and Vaccari, M., "A Far-infrared Spectroscopic Survey of Intermediate Redshift (Ultra) Luminous Infrared Galaxies,” ApJ, 796, 63 (2014).

[66] Díaz-Santos, T., Armus, L., Charmandaris, V., Lu, N., Stierwalt, S., Stacey, G., Malhotra, S., van der Werf, P. P., Howell, J. H., Privon, G. C., Mazzarella, J. M., Goldsmith, P. F., Murphy, E. J., Barcos-Muñoz, L., Linden, S. T., Inami, H., Larson, K. L., Evans, A. S., Appleton, P., Iwasawa, K., Lord, S., Sanders, D. B., and Surace, J. A., “A Herschel/PACS Far-infrared Line Emission Survey of Local Luminous Infrared Galaxies,” ApJ, 846, 32 (2017).

[67] Dwek,. E., and Arendt, R.G., "Dust Masses, Compositions, and Luminosities in the Nuclear Disks and the Diffuse Circumnuclear Medium of Arp 220,” ApJ, 901, 36 (2020). 
[68] Greenberg, R. Wacker, J. F., Hartmann, W. K., Chapman, C. R., "Planetesimals to planets: Numerical simulation of collisional evolution," Icarus, 35, 1-26 (1978).

[69] Kokubo, E., and Ida, S., "Oligarchic Growth of Protoplanets," Icarus, 131, 171-178 (1998).

[70] Ida, S., Y., T., and Okuzumi, S., "Water delivery by pebble accretion to rocky planets in the habitable zones in evolving disks," A\&A, 624, A28 (2019).

[71] Morbidelli, A., Chambers, J., Lunine, J. I., Petit, J. M., Robert, F., Valsecchi, G. B., and Cyr, K. E., "Source regions and time scales for the delivery of water to Earth," Meteorit. Planet. Sci. 35, 1309-1320 (2000).

[72] Owen, T., Bar-Nun, A. \& Kleinfeld, "I. Possible cometary origin of heavy noble gases in the atmospheres of Venus, Earth and Mars," Nature 358, 43-46 (1992).

[73] Shu, F. H., Adams, F. C., and Lizano, S, "Star formation in molecular clouds: observation and theory," ARAA, 25, 23-81 (1987).

[74] Andre, P., Ward-Thompson, D., and Barsony, M., "From Prestellar Cores to Protostars: the Initial Conditions of Star Formation," Protostars and Planets, IV (2000).

[75] Hayashi, C., "Structure of the Solar Nebula, Growth and Decay of Magnetic Fields and the Effects of Magnetic Turbulent Viscocities on the Nebula," Progress of Theoretical Physics Supplement, 70, 35-53 (1981).

[76] Krijt, S., Ciesla, F.J., and Bergin, E. A., "Tracing Water Vapor and Ice During Dust Growth,” ApJ, 833, 285 (2016).

[77] Krijt, S., Bosman, A. D., Zhang, K., Schwarz, K. R., Ciesla, F. J., Bergin, E. A., "CO Depletion in Protoplanetary Disks: A Unified Picture Combining Physical Sequestration and Chemical Processing," ApJ, 899, 134 (2020).

[78] Bergin, E. A., Hogerheijde, M.R., Brinch, C., Fogel, J., Yildiz, U. A., Kristensen, L. E., van Dishoeck, E. F., Bell, T.A., Blake, G. A., Cernicharo, J., Dominik, C., Lis, D., Melnick, G., Neufeld, D., Panic, O., Pearson, J. C., Bachiller, R., Baudry, A, Benedettini, M., Benz, A. O., Bjerkeli, P., Bontemps, S., Braine, J., Bruderer, S., Caselli, P., Codella, C., Daniel, F., di Giorgio, A., M., Doty, S. D., and Encrenaz, P., Fich, M., Fuente, A., Giannini, T., Goicoechea, J. R., de Graauw, Th., Helmich, F., Herczeg, G. J., Herpin, F., Jacq, T., Johnstone, D., Jorgensen, J. K., Larsson, B., Liseau, R., Marseille, M., McCoey, C., Nisini, B., Olberg, M., Parise, B., Plume, R., Risacher, C., Santiago-Garcia, J., Saraceno, P., Shipman, R., Tafalla, M., van Kempen, T. A., Visser, R., Wampfler, S. F., Wyrowski, F., van der Tak, F., Jellema, W., Tielens, A. G. G. M., Hartogh, P., Stutzki, J., and Szczerba, R., "Sensitive limits on the abundance of cold water vapor in the DM Tauri protoplanetary disk," A\&A, 521, L33 (2010).

[79] Hogerheijde, M. R., Bergin, E. A., Brinch, C., Cleeves, L. I., Fogel, J. K. J., Blake, G. A., Dominik, C., Lis, D. C., Melnick, G., Neufeld, D., Panic, O., Pearson, J. C., Kristensen, L., Yildiz, U. A., and van Dishoeck, E. F., "Dection of the Water Reservoir in a Forming Planetary System," Science, 334, 338 (2011).

[80] Long, F., Herczeg, G. J., Harsono, D., Pinilla, P., Tazzari, M., Manara, C. F., Pascucci, I., Cabrit, S., Nisini, B., Johnstone, D., Edwards, S., Salyk, C., Menard., F., Lodato, G., Boehler, Y., Mace, G. N., Liu, Y., Mulders, G. D., Hendler, N., Ragusa, E., Fischer, W. J., Banzatti, A., Rigliaco, E., van de Plas, G., Dipierro, G. Gully-Santiago, M., Lopez-Valdivia, R., "Compact Disks in a High-resolution ALMA Survey of Dust Structures in the Taurus Molecular Cloud," ApJ, 882, 49 (2019).

[81] Chiang, E. and Laughlin, G., "The minimum-mass extrasolar nebula: in situ formation of close-in super-Earths," MNRAS, 431, 3444-3455 (2013).

[82] Du, F., Bergin, E. A., Hogerheijde, M., van Dishoeck, E. F., Blake, G., Bruderer, S., Cleeves, I., Dominik, C., Fedele\}, D., Lis, D. C., Melnick, G., Neufeld, D., Pearson, J., Yildiz, U., "Survey of Cold Water Lines in Protoplanetary Disks: Indications of systematic Volatile Depletion," ApJ, 842, 98 (2017).

[83] van Dishoeck, Kristensen, L. E., Mottram, J. C., Benz, A. O., Bergin, E. A., Caselli, P., Herpin, F., Hogerheijde, M. R., Johnstone, D., Liseau, R., Nisini, B., Tafalla, M., van der Tak, F. F. S., Wyrowski, F., Baudry, A., Benedettini, M., Bjerkeli, P., Blake, G, Braine, J., Bruderer, S., Cabrit, S., Cernicharo, J., Choi, Y., Coutens, A., de Graauw, Th., Dominik, C., Fedele, D., Fich, M., Fuente, A., Furuya, K., Goicoechea, J. R., Harsono, D., Helmich, F. P., Herczeg, G. J., Jacq, T., Karska, A., Kaufman, M., Keto, E., Lamberts, T., Larsson, B., Leurini, S., Lis, D. C., Melnick, G., Neufeld, D., Pagani, L., Persson, M., Shipman, R., Taquet, V., van Kempen, T. A., Walsh, C., Wampfler, S., Yildiz, U., and WISH Team, "Water is star-forming regions: physics and chemistry from clouds to disks as probed by Herschel spectroscopy," A\&A, 648, 57 (2021).

[84] Andrews, S., Huang, J. Perez, L. M., Isella, A., Dullemond, C. P., Kurtovic, N. T., Guzman, V. V., Carpenter, J. M., Wilner, D. J., Zhang, S., Zhu, Z., Birnstiel, T., Bai, X. N., Benisty, M., Hughes, A. M., Oberg, K. I., and Ricci, L.,"The Disk Substrucutres at High Angular Resolution Project (DSHARP). I. Motivation, Sample, Calibration, and Overview," ApJL, 869, L41 (2018).

[85] Zhang, K., Pontoppidan, K. M., Salyk, C., Blake, G. A., "Evidence for a Snow Line beyond the Transitional Radius in the TW Hya Protoplanetary Disk,” ApJ, 766, 82 (2013). 
[86] Fedele, D., Bruderer, S., van Dishoeck, E. F., Hogerheijde, M. R., Panic, O., Brown, J. M., and Henning, T., "DIGIT survey of far-infrared lines from protoplanetary disks. I. [O i], [C ii], OH, $\mathrm{H}_{2} \mathrm{O}$, and $\mathrm{CH}+$," A\&A, 559, A77, (2013).

[87] Lin, D. N. C., Bodenheimer, P., Richardson, D. C., "Orbital migration of the planetary companion of 51 Pegasi to its present location," Nautre, 380, 606-607 (1996).

[88] Rasio, F A., and Ford, E. B., "Dynamical instabilities and the formation of extrasolar planetary systems," Science, 274, 954-956 (1996).

[89] Notsu, S., Nomura, H., Ishimoto, D., Walsh, C., Honda, M., Hirota, T., and Millar, T. J., "Candidate Water Vapor Lines to Locate the $\mathrm{H}_{2} \mathrm{O}$ Snowline through High-dispersion Spectroscopic Observations. I. The Case of a T Tauri Star," ApJ, 827, 113 (2016).

[90] Notsu, S., Nomura, H., Ishimoto, D., Walsh, C., Honda, M., Hirota, T., and Millar, T. J , “Candidate Water Vapor Lines to Locate the H2O Snowline through High-dispersion Spectroscopic Observations. I. The Case of a Herbig Ae Star," ApJ, 836, 118 (2017).

[91] Kennedy, G. M., and Kenyon S. J., "Planet Formation around Stars of Various Masses: The Snow Line and the Frequency of Giant Planets," ApJ, 673, 502-512 (2008).

[92] Fernandes, R. B., Mulders, G. D., Pascucci, I., Mordasini, C., Emsenhuber, A., , "Hints for a Turnover at the Snow Line in the Giant Planet Occurrence Rate," ApJ, 874, 81 (2019).

[93] McClure, M. K., Espaillat, C., Calvet, N., Bergin, E., D'Alessio, P., Watson, D. M., Manoj, P., Sargent, B., and Cleeves, L. I , “Detections of Trans-Neptunian Ice in Protoplanetary Disks,” ApJ, 799, 162 (2015).

[94] Huang, J., Andrews, S. M., Cleeves, L. I., Oberg, K. I., Wilner, D. J., Bai, X., Birnstiel, T., Carpenter, J., Hughes, A. M., Isella, A., Perez, L. M., Ricci, L., Zhu, Z., "CO and Dust Properties in the TW Hya Disk from High-resolution ALMA Observations," ApJ, 852, 122 (2018).

[95] Trapman, L., Facchini, S., Hogerheijde, M. R., van Dishoeck, E. F., Bruderer, S.,, "Gas versus dust sizes of protoplanetary dics: effects of dust evolution," A\&A, 629, A79, (2019).

[96] Bergin, E. A. and Williams, J. P., [The Determination of Protoplanetary Disk Masses], Springer, 1, (2017).

[97] Miotello, A., Bruderer, S., and van Dishoeck, E. F., "Protoplanetary disk masses from CO isotopologue line emission," A\&A, 572, A96 (2014).

[98] Schwarz, K. R., Bergin, E. A., Cleeves, L. I., Zhang, K., Oberg, K. I., Blake, G. A., and Anderson, D., “Unlocking CO Depletion in Protoplanetary Disks. I. The Warm Molecular Layer,” ApJ, 856, 85 (2018).

[99] Zhang, K., Bergin, E. A., Schwarz, K., Krijt, S., and Ciesla, F., "Systematic Variations of CO Gas Abundances with Radius in Gas-rich Protoplanetary Disks," ApJ, 883, 98 (2019).

[100] Veronesi, B., Paneque-Carreno, T., Lodato, G., Testi, Leonardo and $\{\mathrm{P}\{\backslash$ 'e $\}$ rez $\}$, Laura M. and $\{$ Bertin $\}$, Giuseppe and \{Hall \}, C , “A Dynamical Measurement of the Disk Mass n Elias 227," ApJL, 914, L27 (2021).

[101] Trapman, L., Miotello, A., Kama, M., van Dishoeck, E. F., Bruderer, S., "Far-infrared HD emission as a measure of protoplanetary disk mass," A\&A, 605, A69 (2017).

[102] Williams, J. P. and Best, W. M. J., "A Parametric Modeling Approach to Measuring the Gas Masses of Circumstellar Disks," APj, 788, 59 (2014).

[103] Ansdell, M., Williams, J. P., van der Marel, N., Carpenter, J. M., Guidi, G., Hogerheijde, M., Mathews, G. S., Manara, C. F., Miotello, A., Natta, A. Oliveira, I., Tazzari, M., Testi, L., van Dishoeck, E. F., and van Terwisga, S. E., “ALMA Survey of Lupus Protoplanetary Disks. I. Dust and Gas Masses,” ApJ, 828, 46 (2016).

[104] Long, F., Herczeg, G. J., Pascucci, I., Drabek-Maunder, E., Mohanty, S., Testi, L., Apai, D., Hendler, N., Henning, T., Manara, C. F., and Mulders, G. D., "An ALMA Survey of CO Isotopologue Emission from Protoplanetary Disks in Chamaeleon I," ApJ, 844, 99 (2017).

[105] Bergin E. A., Cleeves, L. I., Gorti, U., Zhang, K., Blake, G. A., Green, J. D., Andrews, S. M., Evans, II, N. J., Henning, T., Oberg\}, K., Pontoppidan, K., Qi, C., Salyk, C., and van Dishoeck, E. F., “An old disk still capable of forming a planetary system," Nature, 493, 644-646 (2013).

[106] McClure, M. K., Bergin, E. A., Cleeves, L. I., van Dishoeck, E. F., Blake, G. A., Evans, II, N. J., Green, J. D., Henning, T., Oberg, K. I., Pontoppidan, K. M., and Salyk, C., "Mass Measurements in Protoplanetary Disks from Hydrogen Deuteride," ApJ, 831, 167 (2016).

[107] Schwarz, K. R., Bergin, E. A., Cleeves, L. I., Zhang, K., Oberg, K. I., Blake, G. A., and Anderson, D. E., “The Radial Distribution of $\mathrm{H}_{2}$ and CO in TW Hya as Revealed by Resolved ALMA Observations of CO Isotopologues," ApJ, 823, 91 (2016).

[108] Du, F., Bergin, E. A., and Hogerheijde, M. R. , "Volatile depletion in the TW Hydrae disk atmosphere," ApJL, 807, L32 (2015). 
[109] National Research Council of the National Academies, "New Worlds, New Horizons in Astronomy and Astrophysics," The National Academies Press, Washington DC (2010).

[110] Salinas, V.N., Hogerheijde, M.R., Bergin, E.A., Cleeves, L. I., Brinch, C., Blake, G. A., Lis, D. C., Melnick, G. J., Panic, O., Pearson, J. C., Kristensen, L., Yildiz, U. A., and van Dishoeck, E. F., "First detection of gas-phase ammonia in a planet-forming disk," A\&A, 591, A122-130 (2016).

[111] Phuong, N.T., Chapillon, E., Majumdar, L., Dutrey, A., Guilloteau, S., Pietu, V., Wakelam, V. Diep, P. N., Tang, Y. W., Beck, T., and Bary, J.,"First detection of $\mathrm{H}_{2} \mathrm{~S}$ in a protoplanetary disk," A\&A, 616, L5-11 (2018).

[112] Pilleri, P., Fuente, A., Cernicharo, J., Ossenkopf, V., Berne, O., Gerin, M., Pety, J., Goicoechea, J. R., Rizzo, J. R., Montillaud, J., Gonzalez-Garcia, M., Joblin, C., Le Bourlot, J., Le Petit, F., and Kramer, C., "Herschel/HIFI observations of $\mathrm{CO}, \mathrm{H}_{2} \mathrm{O}$ and $\mathrm{NH}_{3}$ in Monoceros R2," A\&A, 544, A110-122 (2012).

[113] Goicoechea, J.R., Aguado, A., Cuadrado, S., Roncero, O., Pety, J., Bron, E., Fuente, A., Riquelme, D., Chapillon, E., Herrera, C., and Duran, C. A., "Bottlenecks to interstellar sulfur chemistry. Sulfur-bearing hydrides in UVilluminated gas and grains," A\&A 647, A10-35 (2021).

[114] Neill, J.L., Bergin, E.A., Lis, D.C., Schilke, P., Crockett, N. R., Favre, C., Emprechtinger, M., Comito, C., Qin, S.L., Anderson, D. E., Burkhardt, A. M., Chen, J.-H., Harris, B. J., Lord, S. D., McGuire, B. A., McNeill, T. D., Monje, R. R., Phillips, T. G., Steber, A. L., Vasyunina, T., and Yu, S., "Herschel Observations of Extraordinary Sources: Analysis of the Full Herschel/HIFI Molecular Line Survey of Sagittarius B2(N)," ApJ 789, 8-37 (2014).

[115] McGuire, B.A., Brogan, C.L., Hunter, T.R., Remijan, A. J., Blake, G. A., Burkhardt, A. M., Carroll, P. B., van Dishoeck, E. F,. Garrod, R. T., Linnartz, H., Shingledecker, C. N., and Willis, E. R., "First results of an ALMA Band 10 Spectral Line Survey of NGC 6334I: Detections of Glycolaldehyde and New Compact Bipolar Outflow in HDO and CS," ApJL, 863, L35-43 (2018).

[116] Sonnentrucker, P., Neufeld, D.A., Phillips, T.G., Gerin, M., Lis, D. C., de Luca, M., Goicoechea, J. R., Black, J. H., Bell, T. A., Boulanger, F., Cernicharo, J., Coutens, A., Dartois, E., Kazmierczak, M., Encrenaz, P., Falgarone, E., Geballe, T. R., Giesen, T., Godard, B., Goldsmith, P. F., Gry, C., Gupta, H., Hennebelle, P., Herbst, E.. Hily-Blant, P., Joblin, C., Kolos, R., Krelowski, J., Martin-Pintado, J., Menten, K. M., Monje, R., Mookerjea, B., Pearson, J., Perault,, M., and Persson, C. M., Plume, R., Salez, M., Schlemmer, S., Schmidt, M., Stutzki, J., Teyssier, D., and Vastel, C., Yu, S., Caux, E., Gusten, R., Hatch, W. A., Klein, T., Mehdi, I., Morris, P., Ward, J.,S., "Detection of hydrogen fluoride absorption in diffuse molecular clouds with Herschel/HIFI: an ubiquitous tracer of molecular gas," A\&A, 521, L12-17 (2010).

[117] Persson, C.M., Black, J.H., Cernicharo., J., Goicoechea, J. R., Hassel, G. E., Herbst, E., Gerin, M., de Luca, M., Bell, T. A., Coutens, A., Falgarone, E., Goldsmith, P. F., Gupta, H., Kazmierczak, M., Lis, D. C., Mookerjea, B. Neufeld, D. A., Pearson, J., Phillips, T. G., Sonnentrucker, P., Stutzki, J., Vastel, C., Yu, S., Boulanger, F., Dartois, E., Encrenaz, P., Geballe, T. R., Giesen, T., Godard, B., Gry, C., Hennebelle, P., Hily-Blant, P., Joblin, C., Kolos, R., Krelowski, J., Martin-Pintado, J., Menten, K., Monje, R., Perault, M., Plume, R., Salez, M., Schlemmer, S., Schmidt, M., Teyssier, D., Peron, I., Cais, P., Gaufre, P., Cros, A., Ravera, L., Morris, P., Lord, S., Planesas, P., "Nitrogen hydrides in interstellar gas. Herschel/HIFI observations towards G10.6-0.4 (W31C)," A\&A, 521, L45-51 (2010).

[118] Codella, C., Lefloch, B., Ceccarelli, C., Cernicharo, J., Caux, E., Lorenzani, A., Viti, S., Hily-Blant, P., Parise, B., Maret, S., Nisini, B., Caselli, P., Cabrit, S., Pagani, L., Benedettini, M., Boogert, A., Gueth, F., Melnick, G., Neufeld, D., Pacheco, S., Salez, M., Schuster, K., Bacmann, A., Baudry, A., Bell, T., Bergin, E. A., Blake, G., Bottinelli, S., Castets, A., Comito, C., Coutens, A., Crimier, N., Dominik, C., Demyk, K., Encrenaz, P., Falgarone, E., Fuente, A., Gerin, M., Goldsmith, P., Helmich, F., Hennebelle, P., Henning, Th., Herbst, E., Jacq, T., Kahane, C., Kama, M., Klotz, A., Langer, W., Lis, D., Lord, S., Pearson, J., Phillips, T., Saraceno, P., Schilke, P., Tielens, X., van der Tak, F., van der Wiel, M., Vastel, C., Wakelam, V., Walters, A., Wyrowski, F., Yorke, H., Borys, C., Delorme, Y., Kramer, C., Larsson, B., Mehdi, I., Ossenkopf, V., and Stutzki, J., "The CHESS spectral survey of star forming regions: Peering into the protostellar shock L1157-B1," A\&A, 518, L112-117 (2010).

[119] Watson, W. D., "Ion-molecule reactions, molecule formation, and hydrogen-isotope exchange in dense interstellar clouds," ApJ, 188, 35-42 (1974).

[120] Brown, P. D. and Millar, T. J., "Grain-surface formation of multi-deuterated molecules," MNRAS, 240, 25P-29P (1989).

[121] Ceccarelli, C., Dominik, C., Caux, E., Lefloch, B. and Caselli, P., "Discovery of deuterated water in a young protoplanetary disk," ApJ, 631, L81-L84 (2005).

[122] Butner, H. M., Charnley, S. B., Ceccarelli, C., Rodgers, S. D., Pardo, J. R., Parise, B., Cernicharo, J. and Davis, G. R., "Discovery of interstellar heavy water," ApJ, 659(2), L137-L140 (2007). 
[123] Lellouch, E., Bézard, B., Fouchet, T., Feuchtgruber, H., Encrenaz, T. and de Graauw, T., "The deuterium abundance in Jupiter and Saturn from ISO-SWS observations," Astron. Astrophys., 370, 610-622 (2001).

[124] Linsky, J. L., Draine, B. T., Moos, H. W., Jenkins, E. B., Wood, B. E., Oliveira, C., Blair, W. P., Friedman, S. D., Gry, C., Knauth, D., Kruk, J. W., Lacour, S., Lehner, N., Redfield, S., Shull, J. M., Sonneborn, G. and Williger, G. M., "What is the total deuterium abundance in the local galactic disk?," ApJ, 647(2), 1106-1124 (2006).

[125] Peebles, P. J. E., "Primordial Helium Abundance and the Primordial Fireball - Part Two," Ap J, 146, 532 (1966).

[126] Cyburt, R. H., Fields, B. D., Olive, K. A. and Yeh, T.-H., "Big bang nucleosynthesis: Present status," Rev Mod Phys, 88, 015004 (2016).

[127] Epstein, R. I., Lattimer, J. M. and Schramm, D. N., “The origin of deuterium,” Nature, 263, 198 (1976).

[128] Dulieu, F., Amiaud, L., Congiu, E., Fillion, J. -H., Matar, E., Momeni, A., Pirronello, V. and Lemaire, J. L., "Experimental evidence for water formation on interstellar dust grains by hydrogen and oxygen atoms," Astron Astrophys, 512, A30 (5pp) (2010).

[129] Hartogh, P., Lis, D. C., Bockelée-Morvan, D., de Val-Borro, M., Biver, N., Küppers, M., Emprechtinger, M., Bergin, E. A., Crovisier, J., Rengel, M., Moreno, R., Szutowicz, S. and Blake, G. A., "Ocean-like water in the Jupiter-family comet 103P/Hartley 2," Nature, 478, 218-220 (2011).

[130] Lis, D. C., Biver, N., Bockelée-Morvan, D., Hartogh, P., Bergin, E. A., Blake, G. A., Crovisier, J., de Val-Borro, M., Jehin, E., Küppers, M., Manfroid, J., Moreno, R., Rengel, M. and Szutowicz, S., "A Herschel study of D/H in water in the Jupiter-family comet 45P/Honda-Mrkos-Pajdušáková and prospects for $\mathrm{D} / \mathrm{H}$ measurements with CCAT," ApJ Letters, 774(1), L3 (5pp) (2013).

[131] Bockelée-Morvan, D., Calmonte, U., Charnley, S., Duprat, J., Engrand, C., Gicquel, A., Hässig, M., Jehin, E., Kawakita, H., Marty, B., Milam, S., Morse, A., Rousselot, P., Sheridan, S. and Wirström, E., "Cometary isotopic measurements," Sp. Sci. Rev., 197, 47-83 (2015).

[132] Biver, N., Moreno, R., Bockelée-Morvan, D., Sandqvist, Aa., Colom, P., Crovisier, J., Lis, D. C., Boissier, J., Debout, V., Paubert, G., Milam, S., Hjalmarson, A., Lundin, S., Karlsson, T., Battelino, M., Frisk, U., Murtagh, D. and Odin Team, "Isotopic ratios of H, C, N, O, and S in comets C/2012 F6 (Lemmon) and C/2014 Q2 (Lovejoy)," Astron Astrophys, 589, A78 (11pp) (2016).

[133] Bockelée-Morvan, D., Biver, N., Swinyard, B., de Val-Borro, M., Crovisier, J., Hartogh, P., Lis, D. C., Moreno, R., Szutowicz, S., Lellouch, E., Emprechtinger, M., Blake, G. A., Courtin, R., Jarchow, C., Kidger, M., Küppers, M., Rengel, M., Davis, G. R., Fulton, T., Naylor, D., Sidher, S. and Walker, H., "Herschel measurements of the D/H and ${ }^{16} \mathrm{O} /{ }^{18} \mathrm{O}$ ratios in water in the Oort cloud comet C/2009 P1 (Garradd)," Astron Astrophys, 544, L15 (6pp) (2012).

[134] Villanueva, G. L., Mumma, M. J., Bonev, B. P., Di Santi, M. A., Gibb, E. L., Böhnhardt, H. and Lippi, M., “A sensitive search for deuterated water in comet 8P/Tuttle," ApJ, 690, L5-L9 (2009).

[135] Gibb, E. L., Bonev, B. P., DiSanti, M. A., Villanueva, G. L., Paganini, L. and Mumma, M. J., “An infrared search for HDO in Comet D/2012 S1 (ISON) and implications for iSHELL," ApJ, 816, 101 (6pp) (2016).

[136] Paganini, L., Mumma, M. J., Gibb, E. L. and Villanueva, G. L., "Ground-based detection of deuterated water in Comet C/2014 Q2 (Lovejoy) at IR wavelengths," ApJ, 836(2), L25 (7pp) (2017).

[137] Lis, D. C., Bockelée-Morvan, D., Güsten, R., Biver, N., Stutzki, J., Delorme, Y., Durán, C., Wiesemeyer, H. and Okada, Y., "Terrestrial deuterium-to-hydrogen ratio in water in hyperactive comets," Astron. Astrophys., 625, L5 (8pp) (2019)

[138] Biver, N., Moreno, R. and Bockelée-Morvan, D., "HDO in comet 46P/Wirtanen from ALMA observations," in preparation.

[139] Coutens, A., Vastel, C., Caux, E., Ceccarelli, C., Bottinelli, S., Wiesenfeld, L., Faure, A., Scribano, Y. and Kahane, C., "A study of deuterated water in the low-mass protostar IRAS 16293-2422," Astron Astrophys, 539, A132 (12pp) (2012).

[140] Coutens, A., Vastel, C., Cabrit, S., Codella, C., Kristensen, L. E., Ceccarelli, C., van Dishoeck, E. F., Boogert, A. C. A., Bottinelli, S., Castets, A., Caux, E., Comito, C., Demyk, K., Herpin, F., Lefloch, B., McCoey, C., Mottram, J. C., Parise, B., Taquet, V., van der Tak, F. F. S., Visser, R. and Yıldı, U. A., "Deuterated water in the solar-type protostars NGC 1333 IRAS 4A and IRAS 4B,” Astron Astrophys, 560, A39 (16pp) (2013).

[141] Coutens, A., Vastel, C., Hincelin, U., Herbst, E., Lis, D. C., Chavarría, L., Gérin, M., van der Tak, F. F. S., Persson, C. M., Goldsmith, P. F. and Caux, E., "Water deuterium fractionation in the high-mass star-forming region G34.26+0.15 based on Herschel/HIFI data," MNRAS, 445, 1299-1313 (2014).

[142] Persson, M. V., Jørgensen, J. K., van Dishoeck, E. F. and Harsono, D., "The deuterium fractionation of water on solar-system scales in deeply-embedded low-mass protostars," Astron Astrophys, 563, A74 (9pp) (2014). 
[143] Wang, K. -S., van der Tak, F. F. S. and Hogerheijde, M. R., "Kinematics of the inner thousand AU region around the young massive star AFGL 2591-VLA3: a massive disk candidate?," Astron Astrophys, 543, A22 (13pp) (2012).

[144] Emprechtinger, M., Lis, D. C., Rolffs, R., Schilke, P., Monje, R. R., Comito, C., Ceccarelli, C., Neufeld, D. A. and van der Tak, F. F. S., "The abundance, ortho/para ratio, and deuteration of water in the high-mass star-forming region NGC 6334 I," ApJ, 765, 61 (13pp) (2013).

[145] van der Tak, F. F. S., Walmsley, C. M., Herpin, F. and Ceccarelli, C., "Water in the envelopes and disks around young high-mass stars," Astron Astrophys, 447, 1011-1025 (2006).

[146] Helmich, F. P., van Dishoeck, E. F. and Jansen, D. J., "The excitation and abundance of HDO toward W3(OH)/ ${ }_{2} \mathrm{O}$," Astron Astrophys, 313, 657-663 (1996).

[147] van Dishoeck, E. F., Kristensen, L. E., Mottram, J. C., Benz, A. O., Bergin, E. A., Caselli, P., Herpin, F., Hogerheijde, M. R., Johnstone, D., Liseau, R., Nisini, B., Tafalla, M., van der Tak, F. F. S., Wyrowski, F., Baudry, A., Benedettini, M., Bjerkeli, P., Blake, G. A., Braine, J., Bruderer, S., Cabrit, S., Cernicharo, J., Choi, Y., Coutens, A., de Graauw, Th., Dominik, C., Fedele, D., Fich, M., Fuente, A., Furuya, K., Goicoechea, J. R., Harsono, D., Helmich, F. P., Herczeg, G. J., Jacq, T., Karska, A., Kaufman, M., Keto, E., Lamberts, T., Larsson, B., Leurini, S., Lis, D. C., Melnick, G., Neufeld, D., Pagani, L., Persson, M., Shipman, R., Taquet, V., van Kempen, T. A., Walsh, C., Wampfler, S. F., Y1ldiz, U. and WISH Team, "Water in star-forming regions: physics and chemistry from clouds to disks as probed by Herschel spectroscopy," Astron Astrophys, 648, A24 (57pp) (2021).

[148] Bonal, L., Alexander, C. M. O. 'D., Huss, G. R., Nagashima, K., Quirico, E. and Beck, P., "Hydrogen isotopic composition of the water in CR chondrites," Geochim et Cosmochem Acta, 106, 111-133 (2013).

[149] Yang, L., Ciesla, F. J. and Alexander, C. M. O. 'D., "The D/H ratio of water in the solar nebula during its formation and evolution," Icarus, 226(1), 256-267 (2013).

[150] Jacquet, E. and Robert, F., "Water transport in protoplanetary disks and the hydrogen isotopic composition of chondrites," Icarus, 223(2), 722-732 (2013).

[151] Furuya, K., Aikawa, Y., Nomura, H., Hersant, F., and Wakelam, V., "Water in Protoplanetary Disks: Deuteration and Turbulent Mixing," ApJ, 779, 11 (2013).

[152] Pierel, J. D. R., Nixon, C. A., Lellouch, E., Fletcher, L. N., Bjoraker, G. L., Achterberg, R. K., Bézard, B., Hesman, B. E., Irwin, P. G. J. and Flasar, F. M., "D/H ratios on Saturn and Jupiter from Cassini CIRS,” Astron J, 154,178 (2017).

[153] Feuchtgruber, H., Lellouch, E., Orton, G., de Graauw, T., Vandenbussche, B., Swinyard, B., Moreno, R., Jarchow, C., Billebaud, F., Cavalié, T., Sidher, S. and Hartogh, P., "The D/H ratio in the atmospheres of Uranus and Neptune from Herschel-PACS observations," Astron Astrophys, 551, A126 (9pp) (2013).

[154] Meier, R., Owen, T. C., Matthews, H. E., Jewitt, D. C., Bockelee-Morvan, D., Biver, N., Crovisier, J. and Gautier, D., "A determination of the $\mathrm{HDO} / \mathrm{H}_{2} \mathrm{O}$ ratio in Comet C/1995 O1 (Hale-Bopp)," Science, 279(5352), 842 (1998).

[155] Hutsemékers, D., Manfroid, J., Jehin, E., Zucconi, J. -M. and Arpigny, C., "The ${ }^{16} \mathrm{OH} /{ }^{18} \mathrm{OH}$ and OD/OH isotope ratios in comet C/2002 T7 (LINEAR)," Astron Astrophys, 490(3), L31-L34 (2008).

[156] Weaver, H. A., A'Hearn, M. F., Arpigny, C., Combi, M. R., Feldman, P. D., Tozzi, G. -P., Dello Russo, N. and Festou, M. C., "Atomic deuterium emission and the D/H ratio in comets," Asteroids, Comets, Meteors Conf. 2008, LPI Contribution 1405, id. 8216 (2008).

[157] Biver, N., Bockelée-Morvan, D., Crovisier, J., Lis, D. C., Moreno, R., Colom, P., Henry, F., Herpin, F., Paubert, G. and Womack, M., "Radio wavelength molecular observations of comets C/1999 T1 (McNaught-Hartley), C/2001 A2 (LINEAR), C/2000 WM1 (LINEAR) and 153P/Ikeya-Zhang,” Astron Astrophys, 449(3), 1255-1270 (2006).

[158] Eberhardt, P., Dolder, U., Schulte, W., Krankowsky, D., Lammerzahl, P., Hoffman, J. H., Hodges, R. R., Berthelier, J. J. and Illiano, J. M., “The D/H ratio in water from comet P/Halley,” Astron Astrophys, 187, 435-437 (1987).

[159] Altwegg, K., Balsiger, H., Bar-Nun, A., Berthelier, J. J., Bieler, A., Bochsler, P., Briois, C., Calmonte, U., Combi, M., De Keyser, J., Eberhardt, P., Fiethe, B., Fuselier, S., Gasc, S., Gombosi, T. I., Hansen, K. C., Hässig, M., Jäckel, A., Kopp, E., Korth, A., LeRoy, L., Mall, U., Marty, B., Mousis, O., Neefs, E., Owen, T., Rème, H., Rubin, M., Sémon, T., Tzou, C. -Y., Waite, H. and Wurz, P., "67P/Churyumov-Gerasimenko, a Jupiter family comet with a high D/H ratio," Science, 347, id 1261952 (3pp) (2015).

[160] Dello Russo, N., Kawakita, H., Vervack, R. J. and Weaver, H. A., "Emerging trends and a comet taxonomy based on the volatile chemistry measured in thirty comets with high-resolution infrared spectroscopy between 1997 and 2013," Icarus, 278, 301-332 (2016).

[161] Mumma, M. J. and Charnley, S. B., "The chemical composition of comets - emerging taxonomies and natal heritage," Ann Rev Astron Astrophys, 49, 471-524 (2011). 
[162] Morgan, J. S., Burgett, W. and Onaka, P., "The Pan-STARRS Project in 2014,” Proc. SPIE, 9145, 91450Y (18pp) (2014)

[163] Mainzer, A., Bauer, J., Cutri, R. M., Grav, T., Masiero, J., Beck, R., Clarkson, P., Conrow, T., Dailey, J., Eisenhardt, P., Fabinsky, B., Fajardo-Acosta, S., Fowler, J., Gelino, C., Grillmair, C., Heinrichsen, I., Kendall, M., Kirkpatrick, J. Davy, Liu, F., Masci, F., McCallon, H., Nugent, C. R., Papin, M., Rice, E., Royer, D., Ryan, T., Sevilla, P., Sonnett, S., Stevenson, R., Thompson, D. B., Wheelock, S., Wiemer, D., Wittman, M., Wright, E. and Yan, L., "Initial performance of the NEOWISE Reactivation Mission,” ApJ, 792(1), 30 (14pp) (2014).

[164] Bauer, James M., Grav, Tommy, Fernández, Yanga R., Mainzer, A. K., Kramer, Emily A., Masiero, Joseph R., Spahr, Timothy, Nugent, C. R., Stevenson, Rachel A., Meech, Karen J., Cutri, Roc M., Lisse, Carey M., Walker, Russell, Dailey, John W., Rosser, Joshua, Krings, Phillip, Ruecker, Kinjal and Wright, Edward L. and NEOWISE Team, "Debiasing the NEOWISE Cryogenic mission comet populations," Astron J, 154(2), 53 (9pp) (2017).

[165] Hansen, C. J., Esposito, L., Stewart, A. I. F., Colwell, J., Hendrix, A., Pryor, W., Shemansky, D. and West, R., "Enceladus' water vapor plume," Science, 311, 1422-1425 (2006).

[166] Porco, C. C., Helfenstein, P., Thomas, P. C., Ingersoll, A. P., Wisdom, J., West, R., Neukum, G., Denk, T., Wagner, R., Roatsch, T., Kieffer, S., Turtle, E., McEwen, A., Johnson, T. V., Rathbun, J., Veverka, J., Wilson, D., Perry, J., Spitale, J., Brahic, A., Burns, J. A., Del Genio, A. D., Dones, L., Murray, C. D. and Squyres, S., "Cassini observes the active south pole of Enceladus," Science, 311, 1393-1401 (2006).

[167] Waite, J. H., Combi, M. R., Ip, W.-H., Cravens, T. E., McNutt, R. L., Kasprzak, W., Yelle, R., Luhmann, J., Niemann, H., Gell, D., Magee, B., Fletcher, G., Lunine, J. and Tseng, W.-L., "Cassini Ion and Neutral Mass Spectrometer: Enceladus plume composition and structure," Science, 311, 1419-1422 (2006).

[168] Hartogh, P., Lellouch, E., Moreno, R., Bockelée-Morvan, D., Biver, N., Cassidy, T., Rengel, M., Jarchow, C., Cavalié, T., Crovisier, J., Helmich, F. P. and Kidger, M., "Direct detection of the Enceladus water torus with Herschel," Astron Astrophys, 532, L2 (2011).

[169] Waite, J. H., Jr., Lewis, W. S., Magee, B. A., Lunine, J. I., McKinnon, W. B., Glein, C. R., Mousis, O., Young, D. T., Brockwell, T., Westlake, J., Nguyen, M. -J., Teolis, B. D., Niemann, H. B., McNutt, R. L., Jr., Perry, M. and Ip, W. -H., "Liquid water on Enceladus from observations of ammonia and ${ }^{40} \mathrm{Ar}$ in the plume," Nature, 460, 487-490 (2009).

[170] Pollack, J. B., Hubickyj, O., Bodenheimer, P., Lissauer, J. J., Podolak, M. and Greenzweig, Y., "Formation of the Giant Planets by concurrent accretion of solids and gas," Icarus, 124(1), 62-85 (1996).

[171] Ali-Dib, M., Mousis, O., Petit, J.-M. and Lunine, J. I, "The measured compositions of Uranus and Neptune from their formation on the CO ice line," ApJ, 793, 9 (7pp) (2014).

[172] Mousis, O., Aguichine, A., Atkinson, D. H., Atreya, S. K., Cavalié, T., Lunine, J. I., Mandt, K. E. and Ronnet, T., "Key atmospheric signatures for identifying the source reservoirs of volatiles in Uranus and Neptune," SSRv, 216(5), 77 (2020).

[173] Luszcz-Cook, S. H. and de Pater, I., "Constraining the origins of Neptune's carbon monoxide abundance with CARMA millimeter-wave observations" Icarus 222, 379 (2013).

[174] Cavalié, T., Venot, O., Selsis, F., Hersant, F., Hartogh, P. and Leconte, J., "Thermochemistry and vertical mixing in the tropospheres of Uranus and Neptune: How convection inhibition can affect the derivation of deep oxygen abundances," Icarus 291, 1 (2017).

[175] Paganini, L., Villanueva, G. L., Roth, L., Mandell, A. M., Hurford, T. A., Retherford, K. D. and Mumma, M. J., “A measurement of water vapour amid a largely quiescent environment on Europa," Nature Astron, 4, 266-272 (2020).

[176] Jia, X., Kivelson, M. G., Khurana, K. K. and Kurth, W. S., "Evidence of a plume on Europa from Galileo magnetic and plasma wave signatures," Nature Astron, 2, 459 (2018).

[177] Hartogh, P., "First direct detection of the water atmospheres of Ganymede and Callisto," in preparation.

[178] Grasset, O., Dougherty, M. K., Coustenis, A., Bunce, E. J., Erd, C., Titov, D., Blanc, M., Coates, A., Drossart, P., Fletcher, L. N., Hussmann, H., Jaumann, R., Krupp, N., Lebreton, J. -P., Prieto- Ballesteros, O., Tortora, P., Tosi, F. and Van Hoolst, T., "JUpiter ICy moons Explorer (JUICE): An ESA mission to orbit Ganymede and to characterise the Jupiter system," Planet Sp Sci, 78, 1 (2013).

[179] Hartogh, P. and Ilyushin, Y. A.,"A passive low frequency instrument for radio wave sounding the subsurface oceans of the Jovian icy moons: An instrument concept," Planet Sp Sci, 130, 30 (2016).

[180] Soderblom, L. A., Kieffer, S. W., Becker, T. L., Brown, R. H., Cook, A. F., II, Hansen, C. J., Johnson, T. V., Kirk, R. L. and Shoemaker, E. M., "Triton's geyser-like plumes: Discovery and basic characterization,” Science, 250, 410415 (1990).

[181] Yelle, R. V., “Energy balance and plume dynamics in Triton's lower atmosphere,” Icarus, 89, 347 (1991). 
[182] Bjoraker, G. L., Stolovy, S. R., Herter, T. L., Gull, G. E. and Pirger, B. E., "Detection of water after the collision of fragments G and K of Comet Shoemaker-Levy 9 with Jupiter," Icarus, 121(2), 411-421 (1996).

[183] Feuchtgruber, H., Lutz, D., Beintema, D. A., Valentijn, E. A., Bauer, O. H., Boxhoorn, D. R., De Graauw, Th., Haser, L. N., Haerendel, G., Heras, A. M., Katterloher, R. O., Kester, D. J. M., Lahuis, F., Leech, K. J., Morris, P. W., Roelfsema, P. R., Salama, A., Schaeidt, S. G. and Spoon, H. W. W., Vandenbussche, B. and Wieprecht, E., "External supply of oxygen to the atmospheres of the giant planets," Nature, 389, 159-162 (1997).

[184] Coustenis, A., Salama, A., Lellouch, E., Encrenaz, Th., Bjoraker, G. L., Samuelson, R. E., de Graauw, Th., Feuchtgruber, H. and Kessler, M. F., "Evidence for water vapor in Titan's atmosphere from ISO/SWS data," Astron Astrophys, 336, L85-L89 (1998).

[185] Cottini, V., Nixon, C. A., Jennings, D. E., Anderson, C. M., Gorius, N., Bjoraker, G. L., Coustenis, A., Teanby, N. A., Achterberg, R. K., Bézard, B., de Kok, R., Lellouch, E., Irwin, P. G. J., Flasar, F. M. and Bampasidis, G., "Water vapor in Titan's stratosphere from Cassini CIRS far-infrared spectra," Icarus, 220, 855-862 (2012).

[186] Moreno, R., Lellouch, E., Lara, L. M., Feuchtgruber, H., Rengel, M., Hartogh, P. and Courtin, R., "The abundance, vertical distribution and origin of $\mathrm{H}_{2} \mathrm{O}$ in Titan's atmosphere: Herschel observations and photochemical modelling., Icarus, 221, 753-767 (2012).

[187] Griffith, C. A., Penteado, P., Baines, K., Drossart, P., Barnes, J., Bellucci, G., Bibring, J., Brown, R., Buratti, B., Capaccioni, F., Cerroni, P., Clark, R., Combes, M., Coradini, A., Cruikshank, D., Formisano, V., Jaumann, R., Langevin, Y., Matson, D., McCord, T., Mennella, V., Nelson, R., Nicholson, P., Sicardy, B., Sotin, C., Soderblom, L. A. and Kursinski, R., "The Evolution of Titan's Mid-Latitude Clouds," Science, 310, 474 (2005).

[188] Porco, C. C., Baker, E, Barbara, J., Beurle, K., Brahic, A., Burns, J. A., Charnoz, S., Cooper, N., Dawson, D. D., Del Genio, A. D., Denk, T., Dones, L., Dyudina, U., Evans, M. W., Fussner, S., Giese, B., Grazier, K., Helfenstein, P., Ingersoll, A. P., Jacobson, R. A., Johnson, T. V., McEwen, A., Murray, C. D., Neukum, G., Owen, W. M., Perry, J., Roatsch, T., Spitale, J., Squyres, S., Thomas, P., Tiscareno, M., Turtle, E. P., Vasavada, A. R., Veverka, J., Wagner, R. and West, R., "Imaging of Titan from the Cassini spacecraft," Nature, 434, 159 (2005).

[189] Turtle, E. P., Perry, J. E., McEwen, A. S., Del Genio, A. D., Barbara, J., West, R. A., Dawson, D. D. and Porco, C. C., "Cassini imaging of Titan's high-latitude lakes, clouds, and south-polar surface changes," Geophys Res Lett, 36, L02204 (2009).

[190] Turtle, E. P., Del Genio, A. D., Barbara, J. M., Perry, J. E., Schaller, E. L., McEwen, A. S., West, R. A. and Ray, T. L., "Seasonal changes in Titan's meteorology," Geophys Res Lett, 38, L03203 (2011).

[191] Anderson, C. M., Samuelson, R. E., Achterberg, R. K., Barnes, J. W. and Flasar, F. M., "Subsidence-induced methane clouds in Titan's winter polar stratosphere and upper troposphere," Icarus, 243, 129 (2014).

[192] Cavalié, T., Hue, V., Hartogh, P., Moreno, R., Lellouch, E., Feuchtgruber, H., Jarchow, C., Cassidy, T., Fletcher, L. N., Billebaud, F., Dobrijevic, M., Rezac, L., Orton, G. S., Rengel, M., Fouchet, T. and Guerlet, S., "Herschel map of Saturn's stratospheric water, delivered by the plumes of Enceladus," Astron Astrophys, 630, A87 (15pp) (2019).

[193] Bjoraker, G. L., "Cassini/CIRS observations of water vapor in Saturn's stratosphere," in preparation.

[194] Waite, J. H., Perryman, R. S., Perry, M. E., Miller, K. E., Bell, J., Cravens, T. E., Glein, C. R., Grimes, J., Hedman, M., Cuzzi, J., Brockwell, T., Teolis, B., Moore, L., Mitchell, D. G., Persoon, A., Kurth, W. S., Wahlund, J. -E., Morooka, M., Hadid, L. Z., Chocron, S., Walker, J., Nagy, A., Yelle, R., Ledvina, S., Johnson, R., Tseng, W., Tucker, O. J. and Ip, W. -H., "Chemical interactions between Saturn's atmosphere and its rings," Science, 362, 2382 (2018).

[195] Cavalié, T., Feuchtgruber, H., Lellouch, E., de Val-Borro, M., Jarchow, C., Moreno, R., Hartogh, P., Orton, G., Greathouse, T. K., Billebaud, F., Dobrijevic, M., Lara, L. M., González, A. and Sagawa, H., "Spatial distribution of water in the stratosphere of Jupiter from Herschel HIFI and PACS observations," Astron Astrophys, 553, A21 (16pp) (2013).

[196] Küppers, M., O'Rourke, L., Bockelée-Morvan, D., Zakharov, V., Lee, S., von Allmen, P., Carry, B., Teyssier, D., Marston, A., Müller, T., Crovisier, J., Barucci, M. A. and Moreno, R., "Localized sources of water vapour on the dwarf planet (1) Ceres," Nature, 505, 525-527 (2014).

[197] Nathues, A., Hoffmann, M., Schaefer, M., Le Corre, L., Reddy, V., Platz, T., Cloutis, E. A., Christensen, U., Kneissl, T., Li, J. -Y., Mengel, K., Schmedemann, N., Schaefer, T., Russell, C. T., Applin, D. M., Buczkowski, D. L., Izawa, M. R. M., Keller, H. U., O'Brien, D. P., Pieters, C. M., Raymond, C. A., Ripken, J., Schenk, P. M., Schmidt, B. E., Sierks, H., Sykes, M. V., Thangjam, G. S. and Vincent, J. -B., "Sublimation in bright spots on (1) Ceres," Nature, 528, 237-240 (2015).

[198] de Val-Borro, M., Rezac, L., Hartogh, P., Biver, N., Bockelée-Morvan, D., Crovisier, J., Küppers, M., Lis, D. C., Szutowicz, S., Blake, G. A., Emprechtinger, M., Jarchow, C., Jehin, E., Kidger, M., Lara, L. -M., Lellouch, E., 
Moreno, R. and Rengel, M.., "An upper limit for the water outgassing rate of the main-belt comet 176P/LINEAR observed with Herschel/WIFI," Astron Astrophys, 546, L4 (4pp) (2012).

[199] O’Rourke, L., Snodgrass, C., de Val-Borro, M., Biver, N., Bockelée-Morvan, D., Hsieh, H., Teyssier, D., Fernandez, Y., Kueppers, M., Micheli, M. and Hartogh, P., "Determination of an upper limit for the water outgassing rate of main-belt comet P/2012 T1 (Pan-STARRS)," ApJ Letters, 774(1), L13 (4pp) (2013).

[200] Feldman, W. C., Maurice, S., Binder, A. B., Barraclough, B. L., Elphic, R. C. and Lawrence, D. J., "Fluxes of fast and epithermal neutrons from Lunar Prospector: Evidence for water ice at the lunar poles," Science, 281, 1496 (1998).

[201] Pieters, C. M., Goswami, J. N., Clark, R. N., Annadurai, M., Boardman, J., Buratti, B., Combe, J. -P., Dyar, M. D., Green, R., Head, J. W., Hibbitts, C., Hicks, M., Isaacson, P., Klima, R., Kramer, G., Kumar, S., Livo, E., Lundeen, S., Malaret, E., McCord, T., Mustard, J., Nettles, J., Petro, N., Runyon, C., Staid, M., Sunshine, J., Taylor, L. A., Tompkins, S. and Varanasi, P., "Character and spatial distribution of $\mathrm{OH} / \mathrm{H}_{2} \mathrm{O}$ on the surface of the Moon seen by M3 on Chandrayaan-1," Science, 326, 568-572 (2009).

[202] Sunshine, J. M., Farnham, T. L., Feaga, L. M., Groussin, O., Merlin, F., Milliken, R. E. and A'Hearn, M. F., "Temporal and spatial variability of lunar hydration as observed by the Deep Impact spacecraft," Science, 326, 565567 (2009).

[203] Clark, R. N., "Detection of adsorbed water and hydroxyl on the Moon," Science, 326, 562-564 (2009).

[204] Colaprete, A., Schultz, P., Heldmann, J., Wooden, D., Shirley, M., Ennico, K., Hermalyn, B., Marshall, W., Ricco, A., Elphic, R. C., Goldstein, D., Summy, D., Bart, G. D., Asphaug, E., Korycansky, D., Landis, D. and Sollitt, L., "Detection of water in the LCROSS ejecta plume," Science, 330, 463-468 (2010).

[205] Honniball, C. I., Lucey, P. G., Li, S., Shenoy, S., Orlando, T. M., Hibbitts, C. A., Hurley, D. M. and Farrell, W. M., "Molecular water detected on the sunlit Moon by SOFIA," Nature Astron, 5, 121-127 (2021).

[206] Hurley, D. M. and Benna, M., "Simulations of lunar exospheric water events from meteoroid impacts," Planet Sp Sci, 162, 148-156 (2018).

[207] Benna, M., Hurley, D. M., Stubbs, T. J., Mahaffy, P. R. and Elphic, R. C., "Lunar soil hydration constrained by exospheric water liberated by meteoroid impacts.," Nature Geoscience, 12, 333-338 (2019).

[208] Gurwell, M. A., Melnick, G. J., Tolls, V., Bergin, E. A. and Patten, B. M, "SWAS observations of water vapor in the Venus mesosphere," Icarus, 188, 288-304 (2007).

[209] Sandor, B. J. and Clancy, T. R., "Water vapor variations in the Venus mesosphere from microwave spectra," Icarus, 177(1), 129-143 (2005).

[210] Donahue, T. M., Grinspoon, D. H., Hartle, R. E. and Hodges, R. R., Jr., "Ion/neutral escape of hydrogen and deuterium: Evolution of water." In Venus II Geology, Geophysics, Atmosphere and Solar Wind Environment (S. W. Bougher, D. M. Hunten, and R. J. Phillips, Eds.), pp. 585-414. Univ. of Arizona Press, Tucson (1997).

[211] Fedorova, A., Korablev, O., Vandaele, A. -C., Bertaux, J. -L., Belyaev, D., Mahieux, A., Neefs, E., Wilquet, W. V., Drummond, R., Montmessin, F. and Villard, E., "HDO and $\mathrm{H}_{2} \mathrm{O}$ vertical distributions and isotopic ratio in the Venus mesosphere by Solar Occultation at Infrared spectrometer on board Venus Express," J Geophys Res, 113, E00B22 (2008).

[212] Donahue, T. M., "New analysis of hydrogen and deuterium escape from Venus," Icarus, 141(2), 226-235 (1999).

[213] Way, M. J., Del Genio, A. D., Kiang, N. Y., Sohl, L. E., Grinspoon, D. H., Aleinov, I., Kelley, M. and Clune, T., "Was Venus the first habitable world in our solar system?," Geophys Res Lett, 43(16), 8376-8383 (2016).

[214] Bertaux, J.-L., Nevejans, D., Korablev, O., Villard, E., Quémerais, E., Neefs, E., Montmessin, F., Leblanc, F., Dubois, J. P., Dimarellis, E., Hauchecorne, A., Lefèvre, F., Rannou, P., Chaufray, J. Y., Cabane, M., Cernogora, G., Souchon, G., Semelin, F., Reberac, A., Van Ransbeek, E., Berkenbosch, S., Clairquin, R., Muller, C., Forget, F., Hourdin, F., Talagrand, O., Rodin, A., Fedorova, A., Stepanov, A., Vinogradov, I., Kiselev, A., Kalinnikov, Yu., Durry, Georges, Sandel, B., Stern, A. and Gérard, J. C., "SPICAV on Venus Express: Three spectrometers to study the global structure and composition of the Venus atmosphere," Planet Sp Sci, 55(12), 1673-1700 (2007).

[215] Esposito, L. W., Copley, M., Eckert, R., Gates, L., Stewart, A. I. F. and Worden, H., "Sulfur dioxide at the Venus cloud tops, 1978-1986," J Geophys Res, 93, 5267-5276 (1988).

[216] Marcq, E., Bertaux, J-L., Montmessin, F., and Belyaev, D., "Variations of sulphur dioxide at the cloud top of Venus' dynamic atmosphere," Nature Geoscience, 6(1), 25-28 (2013).

[217] Mills, F. P., and Allen, M., "A review of selected issues concerning the chemistry in Venus' middle atmosphere," Planet Sp Sci, 55, 1729-1740 (2007).

[218] Krasnopolsky, V. A., "A photochemical model for the Venus atmosphere at 47-112 km,” Icarus, 218(1), 230-246 (2012). 
[219] Vandaele, A. C., Korablev, O., Belyaev, D., Chamberlain, S., Evdokimova, D., Encrenaz, Th., Esposito, L., Jessup, K. L., Lefèvre, F., Limaye, S., Mahieux, A., Marcq, E., Mills, F. P., Montmessin, F., Parkinson, C. D., Robert, S., Roman, T., Sandor, B., Stolzenbach, A., Wilson, C. and Wilquet, V., "Sulfur dioxide in the Venus atmosphere: I. Vertical distribution and variability," Icarus, 295, 16-33 (2017).

[220] Greaves, J. S., Richards, A. M. S., Bains, W., Rimmer, P. B., Sagawa, H., Clements, D. L., Seager, S., Petkowski, J. J., Sousa-Silva, C., Ranjan, S., Drabek-Maunder, E., Fraser, H. J., Cartwright, A., Mueller-Wodarg, I., Zhan, Z., Friberg, P., Coulson, I., Lee, E. and Hog, J., "Phosphine gas in the cloud decks of Venus," Nature Astron, 234 (2020).

[221] Mogul, R., Limaye, S. S., Way, M. J. and Cordova, J. A., "Venus' mass spectra show signs of disequilibria in the middle clouds," Geophys Res Lett, 48 (2021).

[222] Thompson, M. A., "The statistical reliability of 267-GHz JCMT observations of Venus: no significant evidence for phosphine absorption," MNRAS, 501, L18-L22 (2021).

[223] Villanueva, G. L., Cordiner, M., Irwin, P., De Pater, I., Butler, B., Gurwell, M., Milam, S. N., Nixon, C. A., LuszczCook, S. H., Wilson, C. F., Kofman, V., Liuzzi, G., Faggi, S., Fauchez, T., Lippi, M., Cosentino, R., Thelen, A. E., Moullet, A., Hartogh, P., and Kopparapu, R., "No evidence of phosphine in the atmosphere of Venus by independent analyses," Nature Astron, in press (2021).

[224] Akins, A. B., Lincowski, A. P., Meadows, V. S. and Steffes, P. G., "Complications in the ALMA detection of phosphine at Venus," ApJ Letters, 907(2), L27 (7pp) (2021).

[225] Encrenaz, T., Greathouse, T. K., Marcq, E., Widemann, T., Bézard, B., Fouchet, T., Giles, R., Sagawa, H., Greaves, J., and Sousa-Silva, C., "A stringent upper limit of the $\mathrm{PH}_{3}$ abundance at the cloud top of Venus," Astron Astrophys, 643, L5 (4pp) (2020).

[226] Trompet, L., Robert, S., Mahieux, A., Schmidt, F., Erwin, J. and Vandaele, A. C., "Phosphine in Venus' atmosphere: Detection attempts and upper limits above the cloud top assessed from the SOIR/VEx spectra," Astron Astrophys, 645, L4 (6pp) (2021). 